s.6.

Q3.

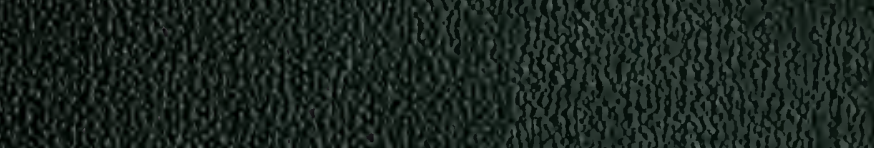

Low 2 .

sor.

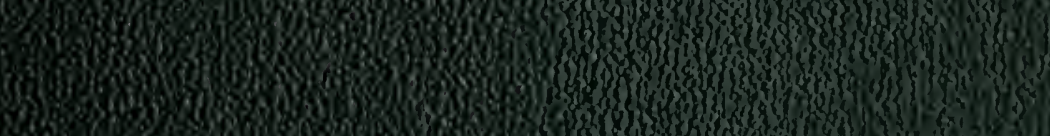
13.70 .

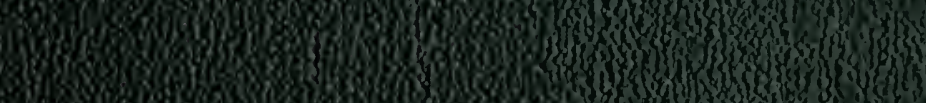

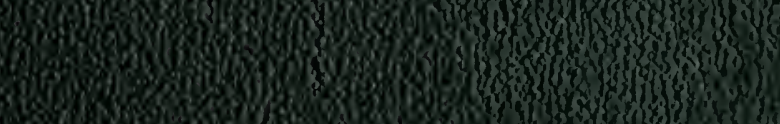

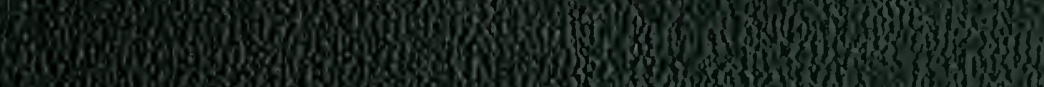

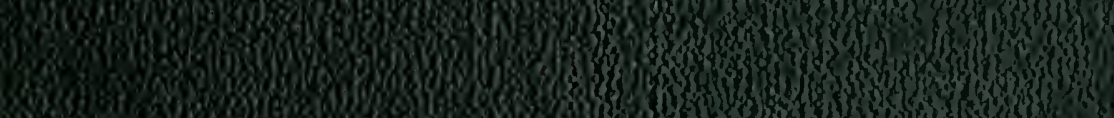

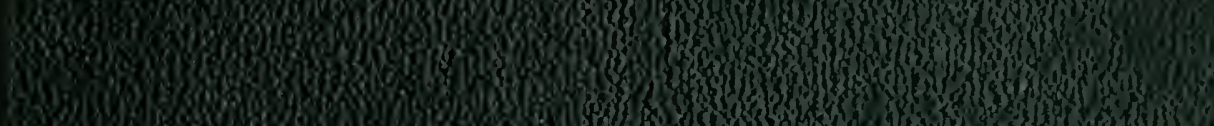

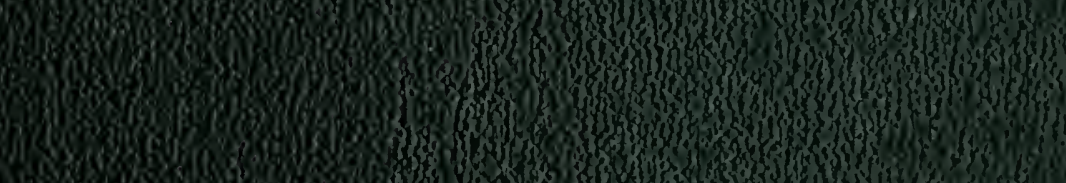
s.5. W. Sow

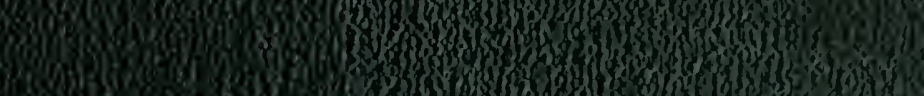

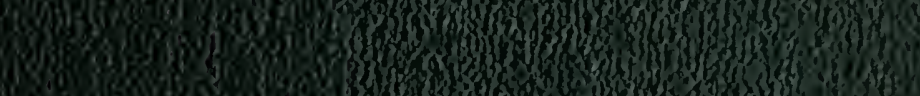

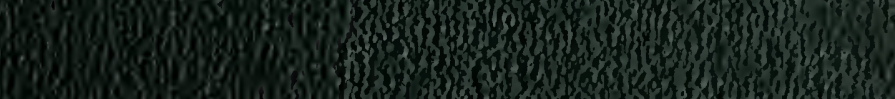

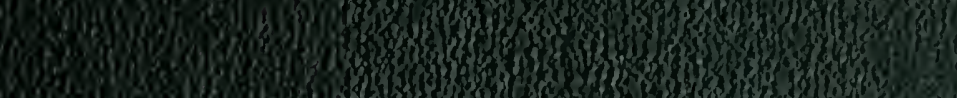
Q3.7. V.8.

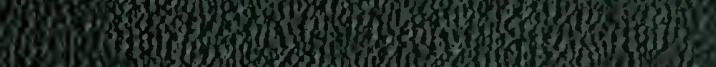

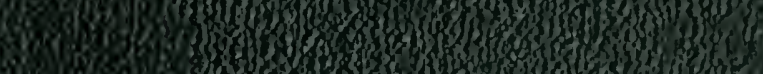
15.

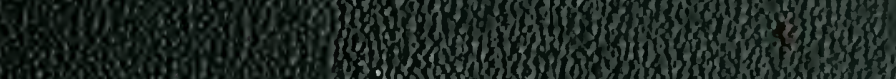

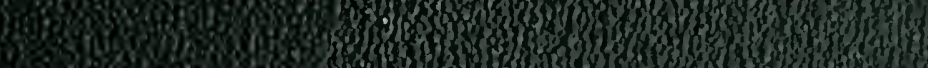
19.5\%

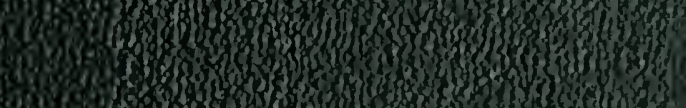

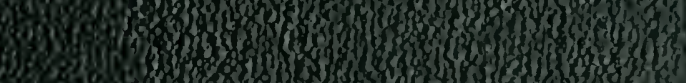




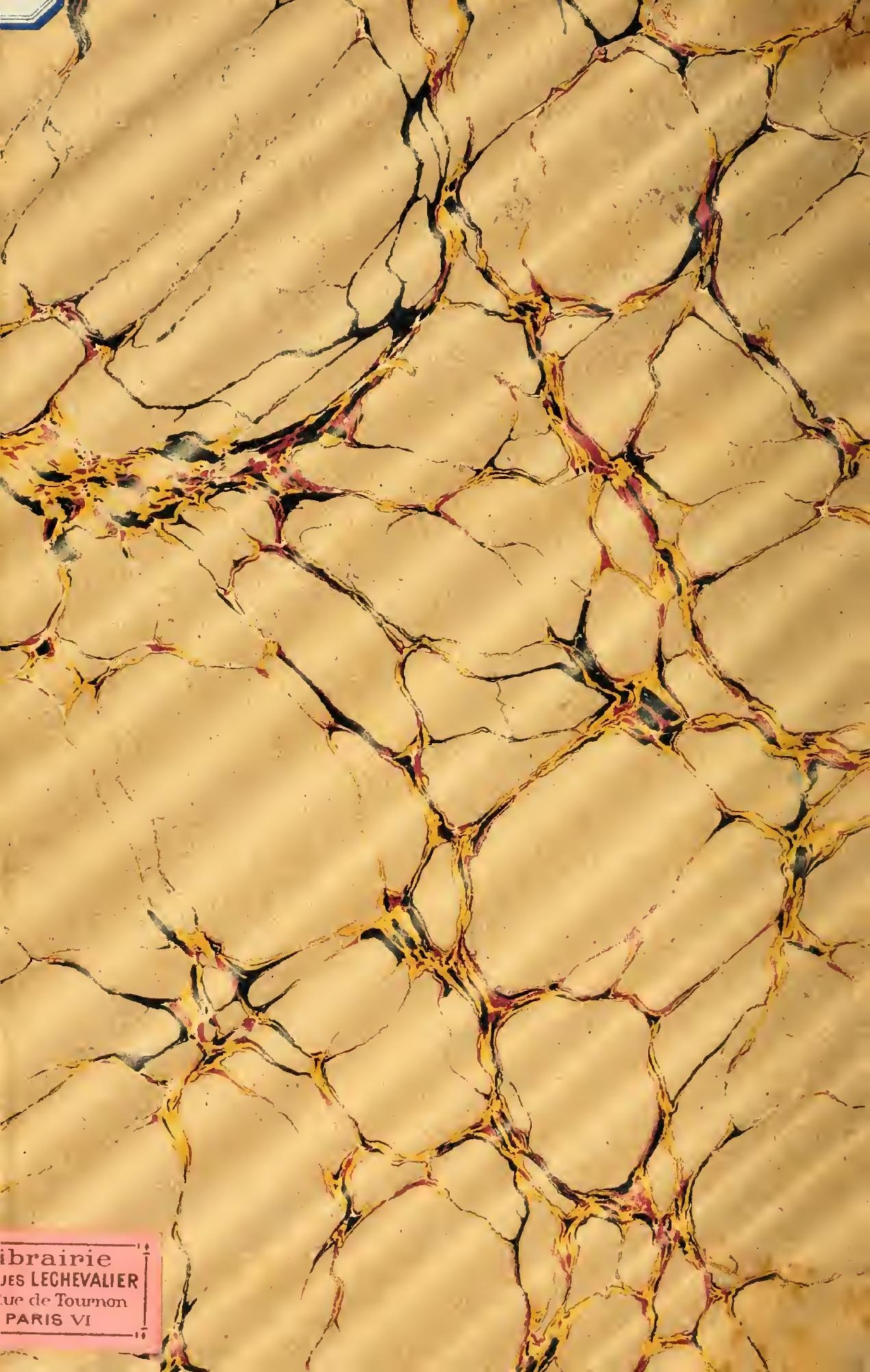




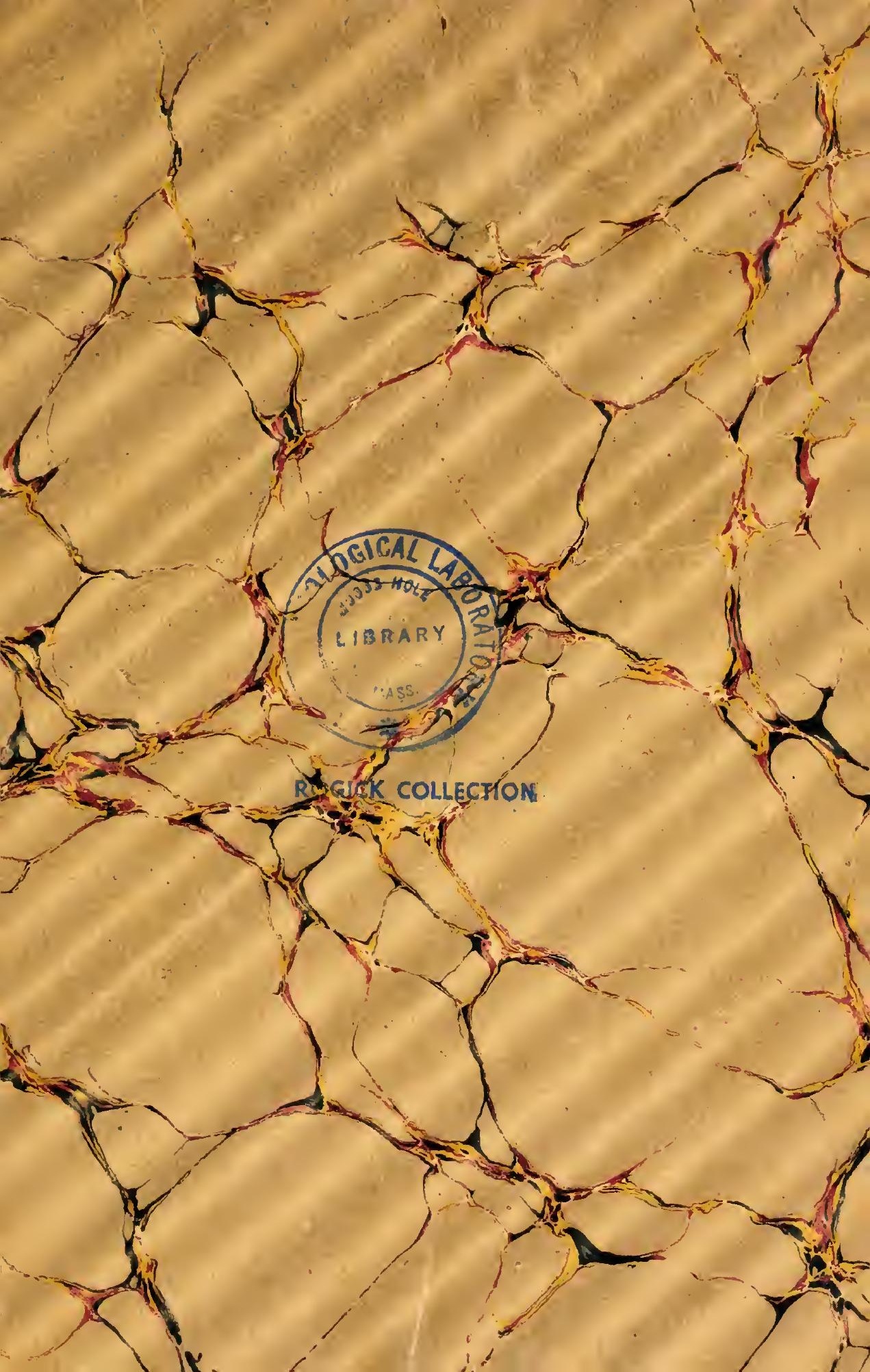





\section{etrary Oorea Pogicter 1955.}

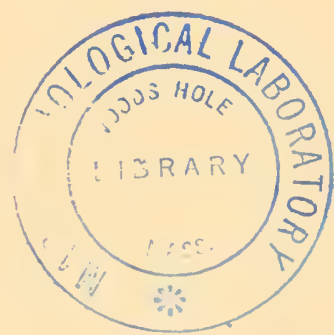

ROGICK COLLECTION 





\section{THESES}

PIESETTES

\section{A LA PACULTE DES SCIENCES IE PARIS}

POL:R OIBTH:NIR

LE GRADE DE DOCTEUR ĖS SCIEXCES NATURLLLES

PAl

\section{LUGIEN JOLIET}

PLICEXCIÉ ḰS SCIENYES NATURELLFS

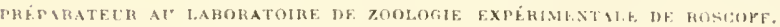

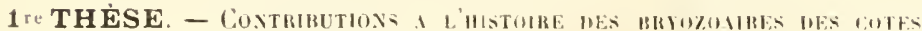
DE FBANCE,

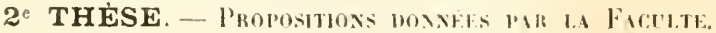

Soutemwes le movemlite revant la commusion d'examen

M.M. IIEBERT, President

DE LACAZE DUTHLES, j ERminatrurs.
DUCHARTRE,

\section{PARIS}

TYPOGRAPHE A. HENNUYER

HL̈E U’A REET, 7 


\section{ACADEMIE DE PARIS}

\section{FAGULPF DE- SGIENGEF DE PARIS}

MII

DOYEN...... MILNE-EDWARDS, Prof. Zoologie, Anatomie, Physiologit comparée. '

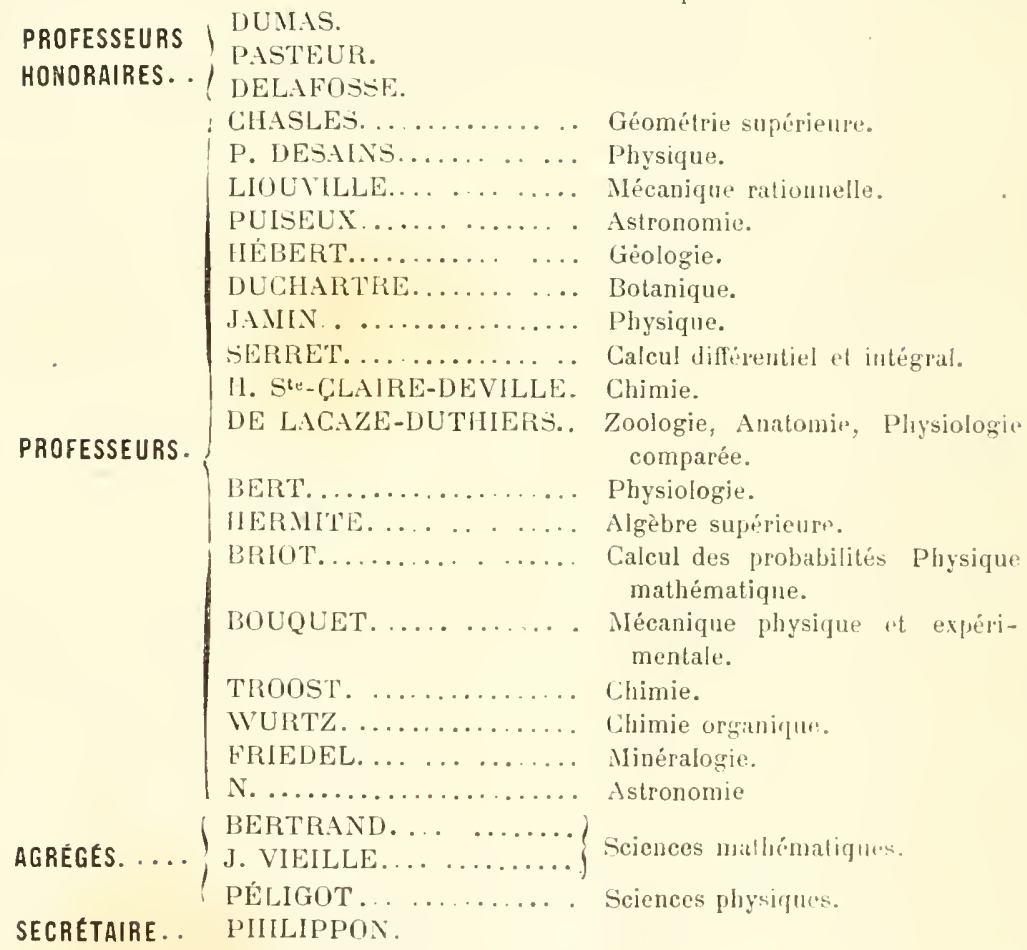




\title{
A II. II. DE LACAZE-IJTHIERS
}

\author{
MENBHE [H: L'INSTITLT

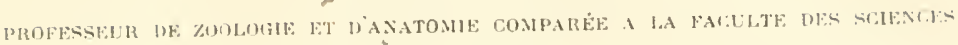 \\ DE PARIS.
}

\section{MONSIEUR ET CHER MAITRE.}

Lorsque, il y a deux ans, séduit par l'attrait de vos leçons et par cet entrain que vous savez si bien communiquer autour de vous, je rous ai demandé de m’admettre dans votre laboratoire, cétait, avant tout, votre méthode de travail que je désirais apprendre de vous.

Alors que j’étais encore étudiant, vous m'avez prodigur et votre temps et vos avis; quand je fus à même de m’engager dans le champ des recherches, c'est encore d'après vos conseils que j’ai concentré mes efforts sur un groupe restreint d'animaux, voulant arriver à posséder de son organisation une connaissance approfondie et contribuer à l'œuvre qui a absorbé une si grande part de votre temps et de vos soins, la description de notre faune française.

C'est alors que vous m'avez placé à Roscoff, dans la position la plus favorable et au milieu de toutes les ressources qu'un zoologiste peut souhaiter.

A plus d'un titre, vous le voyez, cette thèse vous appartient, c'est le fruit de vos enseignements et de vos conseils, veuillez. done en accepter l'hommage comme un tribut de reconnaissince ot, si vous me le permettez, rle l'ajouter comme un témoignagre de respectucuse affection.

L. JOLIET. 



\section{SEGONDE THESE}

\section{PROPOSITIONS DONNÉES PAR LA FAGULTÉ}

Botanrece. - Structure et développement des feuilles.

Fantille des Ombellifères.

GÉoLogre. - Comparer les terrains tertiaires de la Provence et du Languedoc à la série du bassin de Paris.

I'u et approuvé :

Paris, le 13 novembre $1 \$ 77$.

Le Doyen de la Faculté des Sciences, MLNE-EDIVARDS.

Vuet permis d'imprimer :

Paris, le 13 novembre 1877.

Le vice-recteur de l'Académie de Paris,

A. MOURIER. 



\title{
CONTRIBUTIONS
}

$\wedge$

\section{L'HISTOIRE NATURELLE DES BRYOZOAIRES \\ DES COTES DE FRANGE}

\author{
P.AR M. LUCIEN JOLIET \\ Préparateur au laboratoire de Zoologie expérimentale de Roscoif.
}

\section{INTRODUCTION.}

Depuis longlemps déja, on connaît les " Polypes à bras ciliés s et l'on al tracé les principaux traits de leur organisation ". Depuis plus de quarante ans, la classe des Bryozoaires est constituée.

Et cependant l'histoire de ces animaux est aujourd'hui même si incomplétement éclaircie, que l'on discute encore pour savơr sur quel degré de l'échelle zoologique on rloit les placer ².

L'Angleterre, la Suède, l'Allemagne ont fourni, tant sur leur structure que sur leur synonymie, des travaux remarquables dont quelques-uns sont de réritables monuments.

1 Peyssommel et Bernard de Jussieu recomnaissent, en 1741, la nature animale des flustres.

Trembley, en 174', décrit son Polype a panaehe.

Fllis, en 173̈3, dans son Hist. nat. des Corallines, décrit un grand nombre de bryozoaires et reecnnaît la nature des eorps bruns.

Pallas, en 1766, dans son Elenchus, en fait connaître de nombreuses espèces.

Carolini, en 1783 , en étudiant les sérialaires du Pausilippe a eu comine une intuition du bryozoaire, il dit que la sertolara lendinosa (serialaria lendigera) differe de tous les lyydraires jar eeci, que le eorps est rattaché à l'ourerture des loges, il parle du tourbillon et de la trépidation que l'agitation des bras eanse dans l'eau el de la vieaeité aree laquelle ees organes se rétractent.

En 1827, Grunt s'aperçoit que le tube digestif se recourbe $n$ ans?.

En 989, Milne Edwalds et Audouin, aux îles Chasusey, constatent pour la première fois la présence de l'anus dans les Filustres.

2 Voir Reichent, Vergleichende Anatomische Untersuchungen über Zockotryon polucidus, Abl. Ak. Berl., 1870, p. 233. 
Après s'ulre maintenue au premier rang, après aroir énoné et féfini les caractères du groupe, la France, il faut en conrenir, s'est depuis longlemps laissé derancer.

De 1828 à 1890, M. Milnc-Edwards publie ses beaux traraux sur les Flustres, les Eschares, les Crisies, les Tubulipores, ot démontrant l'existence, déterminant la posilion de l’anus dans différents genres des Polypes à bras ciliés, il les sépare nellement des auties Polypes et en fait une classe distincte quil rapproche des Tuniciers.

Entre 1836 et 1839, M. Panl Gervais, fait paraître une série de notes ef un mémoire important sur les Polypes d'eau douce '.

Mais, depuis cette époque jusqu’à ces delniòres années, les noms français sont bien rares sur la liste des auteurs qui ont ajouté quelque chose à l'histoire de ces animanx, et personne ne s'en est occupé spécialement.

11 y a là, ce me semble, une lacune à combler, et c'est arec un vif plaisir que je saisis, l'an dernier, l'oceasion qui me fut bienveiliamment offerte par M. de Lacaze-Duthiers d'y contribuer dans des conditions si exceptionnellement farorables au laboratoire de Roscoff. Lì, deux étés de suite, pendant un séjour lotal de six mois, dans mne localité particulièrement riche, j'aj pu observer à loisis la nature vivante, ayant à ma disposition, comme tous les travailleurs admis dans l’établissement, les embarcations et le persomnel du laboratoire, amplement pourru de tous les moyens d'étude nécessaires aux recherches modernes, recorant régulièrement à Paris, durant l'hiver même, des envois de sujets rivants, enfin soutenn par un maître qui, depuis longues années, a fait tous ses efforts pour accueillir libéralement les travilleurs en vue de réunir les éléments d'une faune des côtes de France, et qui, après avoir consacré une partic de sa vie à en fommir une si large part, n’épargue ni encouragements ni sacrifices pour ceux qui apportent leurs concours à cette ourre. Qne co travail qui lui est dédić lui soit un témoignage de ma reconnaissance.

Si la connaissance des différents types qui constituent un groupe zoologique est nécessaile à qui reut se former une idée exacte de son ensemble, elle n'est pas moins précieuse poul l'obserrateur qui, cherchant à élucider certaines questions relatives à l'anatomie

I Genvals, Amn. fr. el delr. d'anat., t. III, 1839, p. 129. 
ou au déreloppenenf, derra s’adresser de prefórenre à telle ou telle espèce rui présenfe des conditions particulierement favolables à l'élurle.

C"est la une remarque de détail, mais qui a bien son importance dans celle classe d'animanx surtout.

Les loges qui renferment les Polypides au repos sont en effet triesourent opaques, wu tollement chargees d'omements ef d'appendices, gu'on ne reconnait qu'arec la plus grande peine leur contenu.

J'ai ru tel observateur éminent se désespérer de ne pouroir discerner, dans une Sélialaire le T’oḷ̣pide complétement masrué par les ghanulations hrunes de l'endocyste, tandis qu'au travers des parois parfaitemeni translucides d'une espèce fort roisine, je dislingrais sans peine tous les détails de l'organisation.

De mìme telle espece, la Tesicularia spinosa, par excmple, trèslaroable à l'examen anatomique à cause de sa grande traisparence, se piòte mal à lélude du déreloppement des bourgeons.

Fixéc en effet à une assez gramle profondeur d’où on ne la retire qu'irec la dragie, elie ne s'habitue que difficilement à vivie dans nos curetles et sous une faible pression. Au contraire, la Bowerbarlizi imbricata, qui s'attache aus fucus et décourre à chaque maréc, est eximement précieuse à ce point de vue.

J'en ai cultiré longtemps, si je puis m’expriner ainsi, une branche sorlie de la mer et amenée à Paris, é dans une cuvetle plitle que je pourais à tout instant soumeltre an microscope; clle a si bien vón el prospéré durant ciny mois, yue jai pu, pendani cet intervalu, suive la suecession de plusieus's générations de Polypides dans rine mème loge, assister à la formation des corps bruns, el être lémoin du rôle absolument passif qu'ils jonent cher cetle espèce dans le renouvellemeni du Polypide.

Ces corps lruns ont bien intrigué les obserrateurs. Je n’en veux pour preure que les noms multiples sous lesquels on les a dési. gnés ${ }^{1}$.

Dès 17:\%ั, Ellis y a vu les restes des Polypides qui ont successivement habité la loge, Smitt a cru qu'ils renfermaient un cus, Claparède a prétendu depuis qüils n’étaient qu'une sécrétion de !'endo-

1 Corps bruns, dark bodies, mörka kroppar; germ-eapsules, grodd-liapplar, Keim-kapseln, statoblastes, oufs. 
cyste, enfin on a soutenu récemment l'ancienne opinion modifiće en ce qui concerne le mode de formation de res corps.

Quant à leur rôle, on ne s'entend pas mienx à son égard.

Les uns les ont pris pour des oufs; pour d'autres, ce sont encore des xufs, mais d'me nature particulière, ou bien des sortes de statoblastes, masses de matière nutrutive mise en réserve pour serrir au renourellement du Polypide (grodd kappslar, Smilt, Ilincks).

Repiachoff ${ }^{1}$ assure que le corps brun, d'abord distinel du bourgeon, finit par être englobé dans l'iniérieur du tube digestif en roie de développement; enfin, pour d’autres, ce n’est que le résidu des anciens habitants de la loge et rien de plus.

La question est encore pendante, et, malgré les négalions de Nitsche, Hincks ª́fend énergirnuement la théorie de Smitt et les a germcapsules $n$.

L'œuf prend-il naissance dans l'oricelle des cheilostomes, ou bien y achère-t-il simplement de mùrir après être sorti de la zoécie, suivant l'aris de Huxley ${ }^{3}$ ? Les auteur's sont encore partagés d'opinion ì cet égard.

Et ces organes problématiques, aus formes si étranges, qu'on désigne sous les noms de vibracules et d'aviculaires, dans quel but se balancent-ils sans trêre et cuelle est leur signifteation morphologique?

- Le Polypide est-il nine forme de l'individa ou bien un simple organe, un canal digestif (Nahrungschlaurh), comme le veut Claparède?

Que penser de la "métamorphose régressive " décrite aree tant de conviction par cet auteur ${ }^{4}$ ?

Je ne puis faire ici l'énumération de toutes les questions d'anatomie ou d'embryogénie qui restent à élurider et dont la décrmination jetterait certainement de la lumière sur l'histoire de ces animaux, et sur leurs affinités avec les groupes auxquels on a essayé de les raítacher.

Entre elles toutes, l'une des plus dignes d'intérêt sans contredit cst celle du système nerveux colonial.

1 Zur Nahurgeschichte der Chilostom Bryozoer (Zeistschrift für wissen. Zuol., t. XXV1, 1876, p. 139).

2 Hincks, G. J. of micr. sc., t. Xilit, 1873, p. 16.

3 Iluxuter, Note on the Reproluctive Organs of the Chilostome Polyzoa (Q. J. micr. sc., 1836, t. IV, p. 191-192).

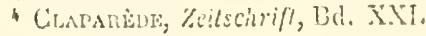


Ouiest-ce que ee eorlon principal, ces lilets tani de fois anastomosés, ce plexus, ees gunglions? Tout cela conslitue-t-il un système nervenz eomme le plus grand nombre lat'fine, est-ce un systeme jrrigatoire, on bien nest-ce rien de semblable, comme on le dit d'allute patle?

Quelle cul est enfin la véritable nature?

Ce sujet comportant des développements assez étendus, nous te réserverous pour la fin de notre travail et nous commencerons par ćludier la question intéressante el bien limitéc du ròle et de la nature du ('orps brun, prenant pour regle de rendre compte des faits aree firálité, de ne les domner que pour ee quils sont, el surtout de ne point les confondre avec les interprétations auxquelles on peut se liver sur leur compte.

\section{GHAPITRE 1.}

DU ROLE ET DE L'OMGINE DES CORPS BRUNS.

Quand on examine à la loupe une branche l'un de nos Bryozoaires marins, d'une Bugule, par exemple, on remarque facilement qu'elle est comme piquetée d'une multitude de petits points de couleur sombre.

Vient-on à en porter un fragment sous le microscope et à l'examiner it un faible grossissement, on reconmait que celte apparence est due à ce que la plupart desloges dépourvues de Polypide contiennent chacune ordinairement un, quelquefois deux petits amas ovoïdes d'un brun plus on moins foncé.

On a donné à ces corps bien des noms, mais daus l'ignorance où l'on est encore de leur véritable nature, on s'accorde à les désigner le plus souvent par la simple appellation de corps bruns.

Le corps brun n'occupe pas dans la loge une place précise; le plus sourent, il se trouve vers le fond, mais on peut le voir sur les côtés ou mème presique au sommet. Il y a des espèces, telles que l'Eucratea chelata, où il est fort rare d'en rencontrer deux à la fois dans une mème loge. Il en est, an contraire, comme la Bowerbankia imbricata, dans lescrnelles on en peut trouver jusqu'à trois.

Chez plusieurs, enfin, on ne roit jamais un corps brun dans une loge pomrne d'un Polypide, tam lis chu'ailleurs la coexistence de l'un 
et de l'autre dans une mème zoécie est chose fréquente et même habituelle.

Le plus sourent, et spécialement chez les espèces où l'on ne trouve qu'un corps brun par loge, le seul rapport constant que celui-ci ait arec ce qui l'entoure, e'est qu'il est en relation avec les branches de ce réseau transparent qu'on a nommé système neveux colonial.

Elles lui serrent en quelque sorte d'amarres et le maintiennent suspendu an milieu de la loge; ailleurs, et plus particulièrement là où phusieurs corps bruns se trourent dans la même zoérie, il y en a un ou deux de relégués contre les parois de l’endocyste el qui ne paraissent plus rattachés au réseau dont je viens de parler ?

Relativement à la zoécie, le corps brun est alssez roluminenx; à l'élat parlatit, e'est-i-dire comme nous le verrons plus loin, lorsfu'il est arrivé à sa taille minima, il peut mesurer jusqu'au quart ou jusqu'au tiors du diamètre transversal de la loge.

Si nous l'examinons sous un grossissement plus fort (environ 300 diamètres), il se montre composé d’um amas de petils grumeanx de matière granuleuse brune sams structure, enfermés dans une envelope générale transparente anhiste doni l'épaisseur augmente arec l'àge.

Par sa présence dans la plupart des loges de la presqu'universalité des Bryozoaires marins el de plusicurs especes d'cau douce ${ }^{2}$, le corps brun semble avoir me importance considérable. Quelle est done sa nature? Quelle est son origine, quel est son rô]e dims l'économie des Bryozoaires?

Je ne sais si cela tient à la difficulté, à la complexité de la matière, ou simplement an grand nombre l'observateurs qui se sont occupés des Bryozoaires, mais pen do sujets ont été, je crois, l'objet de plus de controverses, et, sins avoir sans donte épuisé totalement la bibliographie très-étendue qui se rattache à cetle question, je n’ai pas relevé parmi les auteurs qui l’ont traitée moins de huit opinions différentes auxquelles je serai bién obligé d'en ajouter une neuvième.

1 Pl. XI, fig. ö, et pl. XIII, fig. 1.

2 Nitsche a signalé les corps bruns chez l'Aleyonella fungosa (Zeilschr. f. wissens. Zool, 1. XX11, \%. 470). D'autre part, Allman a déerit (Freshuat. Polyz., p. 40) et liguré (itid., pl. IV, tig. 10, pl. VII, fig. 9) des statoblastes " d'une nature particulime " qui athierent à l'endocyste, qu'il n’a pas vu germer et dont l'anneau est sans structure. A n'en pas douter ce sont des corps bruns. 
Passons en rerue successirement les diverses opinions des auteurs, non pas dans l'ordre chronologique, ee qui n'aurait qu'un intérêt d'arehćologic, mais en grpoupant ensemble cel'es qui se ressemblent le plus : nous examinerons ensuite les laits dans la nature même, ils moliveront les conchusions que nous aurons à en tirer, enfin nous chereherons à discuter les observations de nos devaneiers et à les eoncilier aree notre propre minière de roir.

Voici d'aboril une série d'observateurs qui ont pris les corps bruns pour de véritables reufs:

Thompson ', en se fondant sur la position du corps brun (dans la Vesicularia imbricata) el sur sa persistance après la mort du Polypide, croit pouroir l'assimiler à un œuf ou à un ovaire.

Farre ${ }^{2}$ roit d'abord dans ce corps le résidu de la décomposition (decay) du Polypide, mais sa structure granuleuse, la membrane qui l'enveloppe et sa longue durée sont autant de raisons qui lui font penser qu'il a quelque rapport avec les fonctions de reproduction et doit ĉtre regardé comme un oraire ou comme un ceuf en voie de maturation.

Nordman ${ }^{3} \mathrm{y}$ yoit aussi un auf.

Yan Beneden ", qui a vu dans l'Halodactyle diaphane les véritables œufs, est cependint disposé à regarder les corps bruns comme "le commencement de l'wuf ", et il les tigure comme tels.

Lovén ${ }^{5}$ prétend les avoir vus, dans Ia Valkeria cuscuta, se transformer en larves ciliées.

Redfern ${ }^{6}$ compare les corps bruns aux statoblastes des Bryozoaires d'eau douce.

Allman adopte la même opinion.

Plus tard, Claparède T rappelle cette hypothèse si séduisante et regrette de n'y pouvoir adhérer. Ses observations l'ont en effet conduit à regarder le corps brun comme un produit de séerétion de

I Thompsos, Zool. lle's. and lllustr., mém. V, 1830.

a Farre, On the Siructure of Ciliobranchiate Polypi (Philos. Transacl., 1837, art. BoweriBadia DESA;

3 Nordux, Voyage de Demidoff, 1840, t. III, p. 702, note.

+ VAx Baxedex, Recherches sur les Bryozoaires qui habitent la côte d'Ostende (extr. Nouv. Mèn. dcad. Brux., p. 36, H. V, f, 4e mém.).

5 Lovex, Arsterallelse, 18:40-42, p. 366.

6 Reurers, Flustreila llispida (Q. J. micr. sc., 1. VI, p. 96, 18.3s).

${ }^{7}$ Chapsrède, Beiträge zu Anat. und Entwickelungeschichte der Seebryozoen (Zeilschrift f. wissens. Zool., t. XXI, p. 130 et suir.). 
l'endocyste. Il n'y peut pas voir le principe d'un nouveau Polypide et accompagne d'ailleur's ces rues d'une théorie qui lui est toute particulière sur la métamorphose régressive des Polypides el sur laquelle nous aurons ì revenir.

Hincks ${ }^{1}$ soutient que le corps brun, loin d'ètre le résidu du Polypide flétri, est une formation toute spéciale produite aux dépens de celui-ci par un étranglement survenu vers le milieu du cxeum stomacal, et qui en détacherait l'extrémité inférieure.

Quant à la destinée ultérieure du corps ainsi formé, le même auteur, soutenant chaudement Smitt enntre Nilsche, afirme de la manière la plus positive qu'il sert d'origine à un nouveau Polypide. Traduisant l'appellation de Smitt, il le désigne sous le nom de germcapsule et essaye d’établir que le renourellement du Polypide dans la zoécie adulte peut s'effectuer de deux manières:

$1^{\circ}$ Par formation d'une germ-capsule;

$2^{\circ}$ Par gemmation de l'endoeyste.

L'opinion de Smitt ${ }^{2}$, entourée el appuyée par un grand nombre d'obserrations excellentes, a fait école, on peut le dire; beaucoup d'obserrateur's ont adopté les rues de l'auteur suédois, et le mot qu'il a créé de groddkapslar, pour désigner les corps bruns, a été traduit dans toutes les langues.

Sans bien préciser leur origine, il les considère, soit comme de véritables corps reproducteurs se transformant directement en de nouveaux Polypides, soit comme des amas de matière nutrilive mis en réserve pour servir au développement d'un ceuf qui naîtrait dans leur intérieur. Tout en décrivant la gemmation de l'endocyste, il affirme que dans la plupart des cas (ses olservations portent surtout sur des cheilostomes et cyclostomes), le Polypide se développe aux dépens du corps brun.

Toute opposéc est la manière de voir de Nitsche ${ }^{3}$, qui ne veut reconnaître dans le corps brun que le reste du Polypide flétri, un résidu complétement inerte et incapable de nourrir un nouveau polypide ou de lui servir d'origine. Cette opinion est appuyée de preuves excellentes et de nombreuses figures; elle avait d'ailleurs été déjà

\footnotetext{
1 Rev. TH. lfrxcks, Note on Dr Nitsche's paper (Q.J. micr. sc., 1. XI, 1S71, p. 235 ; t. XIII, 1873, p. 16, pl. II, fig. 7, 6 et 5).

2 SnitT, Om Ilafsbryozoernas Utveckling och Fellkropar.

${ }^{3}$ Nitscir", Beitroge zur Kenlniss der Bryozoen_(Zeitschr. f. wissens. Zool., t. XXI, p. 46 ' et suiv.).
} 
arancée par les anciens observateurs, par Grant et mòme par Ellis.

Tout récemment enfin, Repiachofl' ' vient d’introduire dans le débat de nourelles observations et de nourelles rues.

Pour lui, le bourgeon naît le plus souvent de l'endocyste, mais il finit tòt ou lard par se rapprocher du corps brun; il l'enghobe alor's lans une cavité dui deviendra plus tard l'estomac, et s'en sert commo d'une nourriture pour acherer son développement.

II tigure cet englobement dins lis Tendra zostericole et dans une espèce de Lepralia. Enfin, il compare le corps brun à une sorte de vitellus et ne dit rien d'ailleurs sur son origine.

Abstraction faite des hypotheses qui ne sont plus admises en ancune manière, je résumerai les différentes opinions qui ont cours aujouril'hui dans le tableau suivant :

Le corps lorun est envisagé :

$1^{\circ}$ Quant à son origine : $a$, comme un produit de sécrétion (Claparède) ; $b$, comme une formation spéciale détachéc de l’estomac du Polypide virant (Ilincks); c, comme le résidu du Polypide flétri (Nitsche):

qo Quant à sa destinće ultérieure : $a$, comme un amas de matière inerte, un résidu incapable de virre; $b$, comme un corps vivant servant au renouvellement du Polypide.

Nous voyons par là que deux questions sont encore en litige.

Quelle est l'origine du corps brun?

Quelle est sa destinée ultérieure?

Commençons par aborder la première.

\section{\$. De l'origine des corps bruns.}

Parmi les auteurs dont je riens de citer les opinions touchant l'origine des corps bruns, tous nont pas, il faut le dire, basé la leur sur des observations direetes, patientes et suiries, faites sur des animaux rivants, et la plupart l'ont plutòt présentée, soit comme une simple supposition, soit comme une conclasion tirée de fatits plus ou moins ṫloịnés.

11 me parait impossible qu'un obserrateur soigneux, s̈il s'attache à suirre, comme je l’ai l'ait, jour par jour, pentant une on plusieurs

' Repichoff, Zur Naturgeschichte der Chilostomen Bryozoen (Zeilschr. f. wissens. Zool., t. X.XVI, 1876j. 
semaines, ce qui se passe dans une même logge, n’arrive pas à reconnaitre forcément que le corps brun n'est pas autre ehose que le résidu d'un Polypide llétri.

Les excellentes observations de Nitsehe ${ }^{1}$ et les planches qui les accompagnent me semblent aroir déjà élabli le fait surabondamment pour lat Flustra membranacea.

Cependant comme on a depuis éleré des objections contre ses conclusions, comme on a contesté surtout leur aptitude à s'appliquer alux antres bryozoaires, il me parait nécessaire de les appuyer par de nouvelies oloservations.

Que l'on reuilte done bien jeter les yeux sur la planche VIII qui accompagne ce mémoire.

La première série de figures représente deux loges appartenant à un rameau de Bugula flabellata que j'arais apporté de Roscofl en automne dernier, qui s'est déreloppé à Paris, dans un petit flacon l'eau de mer, vers la fin de l'hiver, el que j'ai rapporté au printemps encore vivant dans son pays natal.

Ces deux loges oceupent l'extrémité de la hranche, sont par conséquent nouvellement formées et ne contiennent ancun corps brun, mais bien deux Polypides E et F, qui sont le 16 janvier en pleine vigneur. On les roit fréquemment s'épanouir et surtout dans le Polypide $\mathrm{F}$ qui parait plus àgé, les granules bruns fournent virement dans le rectum sous l'action des eils ribratiles.

Le 17, E s'épanouit fréquemment, mais F ne sort gnère de sa loge.

Le 23 , E est encore rivant, mais sa eouleur est devenue plus foncée. Quant ì F il a disparu, on plutôt il s'est transfomé en une masse globuleuse de coulenr brune.

Le 26 , le globule brun $F$ a diminué de rolume et sa couleur est derenue plus sombre; (quant à Li it s'est réduit en une masse vroüle rolumineuse d'un brun clair.

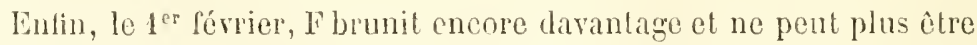
distinguć des corps bruns qui oceupent les loges inférieures. E luimême on est presque au même point, ef il y est arrivé tout à fait peu te jomrs apròs.

Cette observalion est, je pense, suffisante pour démontrer que dans

1 Mitsc!n, heitr. zur Kentniss der Bryozoen (Zeitschr. f. wiss. Zool., t.1XX1, p. 467, 1). XXXit, fig. 1). 
la Buyulu fabellutu, "quand le Polypide se flétrit, il passe à l'état do corps brun el tout entier à l'ćlal de corps brum.

J'ai dans mes eartons une série d'observations tontes semblables à celle-ci, faites dans les nèmes conditions sur la liugula avirularia; le défaut de place m’empèche de la publier, elle n’ajouterail d’ailleurs que peu de chose aux résultats fournis par colle dont je viens de rendre comple. Notons cependant en passant un fait relatif an mode particulier dont se forment les eorps bruns dans les bugules, c'est la rapidité aree laquelle dans l'espace d'une nuit un lolypide, qui semblait encore vigoureux, se transforme totalement en une milsse globuleuse de matière anorphe qui palsse à l'état de eorps brun par diminution graduelle de volume.

Voici maintenant sur la mẻme planche VIII une denxième série de figures qui représentent les mèmes faits dans la Bowerbanlin imbricata; l'explication des planehes me dispense d'entrer de nouveau dans le délail des transformations. Remarquons toutefois que si le terme final est, comme dans les Bugules, un corps brun, le mode suivant lequel il est atteint est différent.

Le premier indice de la déerépitude du Polypide est un certain vague que l'on remarque dans le contour des bras, qui auparavant étrient nets, rigides et placés parallèlement dans leur gainne.

Puis la partie terminale de l'eetoeyste de la loge yui étail invaginée se déroule lentement; peu à peu la collerette (Borstenlirantz, collare setorum) fitit saillie jusqu'à ce que sen point d'insertion soit au ni veau de la surface de la loge, elle pend alors au dehor's comme une sorte de plumet, tandis qu'à l'intérieur les parties se sont désorganisées leutement et lentement affaissées l'une sur l'autre. Souvent dins la Valkerin ruscuta comme dans la Borerbantia imbricata, alor's que les bras ont complétement disparu, l'estomace se contrate cueore, le funicule se rétracte et se détend, les cils vibraliles agitent encore les granules bruns dans le rectum; l'intestin finit enfin par se coller ì l'estomac et le tout se fond en une masse brune que surmonte une tache plus claire, reste des bras et des muscles pariéto-raginaux.

Tout cela finit habitueltement par se confondre en un tout homogène; quelquefois cependant, notamment dans la Boverbankiq imbricata, on reconnait encore longtemps après dans le corpss brun la place vì les restes du gésier el destentacules se sont sondés à ecux de l'estomate.

Je pontrais encore décrire la formation do corps brun dans le Sareo- 
chitum polyoum, dans le Membranipora membranacea, dans la Lepralia gramifera; mais ce serait presque me répéter, j'aurai oceasion de revenir sur son mode de production dans la Bicellaria ciliata. Qu'il me suffise de dire pour l'instant que la formation des corps bruns dans les nombreuses espèces où je l'ai examinée s'est toujours montrée à moi sous deux aspects que j'appellerai l'un, l'aspect bugule, et l'autre, l'aspect vésiculaire.

Dans le premier, qui est le moins fréquent, le Polypide perd subitement toute organisation pour se résoudre en une masse de substance amorphe qui n'a plus qu'à diminuer de volume pour devenir un col'ps brun.

Dans le second la vie ne parât abandonner que successirement les diverses parties, la destruction est plus lente, mais le résultat final est le même.

Dans tous les cas, le Polypide flétri se résout intégralement en un corps brun suspendu comme lui au funicule.

Que faut-il done penser de la théorie de Hincks ${ }^{1}$ ?

A direrses reprises l'éminent auteur affirme de la manière la plus formelle que le corps brun n'est en ancune manière le reste du Polypide ftétri, "in no true sense the remains of the decayig Polypide", mais un colps tout spécial dont la formation se fait d'une manière constante et bien définie.

Il décrit et représente cette formation de la manière suivante :

A un certain moment de l'existence du Polýpide un étranglement se produit en un point du cecum stomacal et en sépare nn corps oroïle creur qui reste longtemps en communication avec lui par un étroit canal. Celui-ci derient de plus et plus long et resserré à mesure que l'étranglement s'accuse davantage, il finit sans doute par se rompre, et le corps ovö̈de ainsi séparé du Polypide constitue un corps brun. Celle description est appuyée de deux figures dessinées d'après la Bicellaria ciliata et qui seraient absolument démonstratives si, comme les observations même qu'elles soutiennent, elles ne prêtaient largement ì la critique.

Notons d'ahord que, bien qu'il regarde la séparation du corps brun d'arec le Polypirle comme un fait positif, Hincks atroue n'en aroil jamais ćté témoin. Or, comme il me sera facile de le prouver tout à l'heure, cette séparation nia jamais lieu même dans la Bicellaria

IIncks, Q. J. micr. sc., t. XI, 1871, p. 233̈; ibid., t. XIII, 1873, p. 16, pl. II. 
ciliata que j’ai tenu à observer particulièrensent pour me mieux rendre compte de la raleur' de l'obserration de l'antenr anglais.

En deuxième lien, après la séparation supposée de ee corps, que derient le polypide dont il dérive et auquel il était relié tout à l'heure? Hincks ne nous en dit rien, et cela serail pourtant nécessaire; quand nous sarons que la vie du Polypide est de courte durée el qu'en mourant il doit laisser dans la loge quelque reste de sil substance.

Enfin renons au fiil. Est-il rai qu’à un moment donné il se produise dans les parois du cacum stomacal un élranglement réritable qui tende à en détacher l'extrémité inférieure?

Dans l’immense majorité des Bryozoaires, les Vésiculaires, les Flustres, les Lepralia et autres, il n'y a rien, absolument rien de semblable et le corps brun se forme suirant le mode que j’ai exposé aree détail un peu plus haut.

Si done le fait que Hincks a décrit est particulier à une ou deux espèces il ne peut pas serrir de base à une théorie générale sur la formation des corps bruns. Mais, d'ailleurs, est-il exart en lui-mème? Examinons les espèces mêmes d'après lesquelles Hincks a dessiné ses figares et posons-nous cette question : la Bicellaria ciliata diffère-t-elle à cet égard de toutes les espèces connues?

Je l'ai étudiée bien longtemps et bien sourent, je n’ai pas eu de peine à reconnailtre dans le cæcum du Polypide cette apparence de constriction dont arguë l'auteur anglais, mais je ne puis l'interpréter de la même manière ni reconnaître pour exacle la figure qu’il en donne.

Il est très-érident et facile à constater que rers te bas du cxecum stomacal la matière brune qui remplit la carité de cet organe semble passer à trarers une sorte de détroit ef qu'en ce point elle est beaucoup plus resserrée qu’en dessus et au-dessous; il est encore rai que la partie inférieure du ceceum ainsi séparée par un défilé de la partie supérieure ressemble assez à une boule brune qui serait suspendue à l'estomac par un canal étroit.

Cette apparence est d'autant plus frappante que les parois intestinales étant très-transparentes, on peut croire qu'elles subissent ellesmêmes l'ćtranglement; c'est ce que représente la figure de Hinclis que je reproduis ${ }^{1}$. Mais, pour peu qu'on y regarde de plus près, on rerra que le contour extérieur du cacum est parfaitement continu, ne subit 
aucun ćtranglement et que si la matière brune intérieure est resserrée en un point, cela tient non pas à me inflexion, mais à un épaississement des parois stomacales à ce niveau, à une sorte de saillie, de bourrelet amnulaire qu' elles forment à l'intérieur et qui détermine à tons les moments de la vie du Polypide et dés son plus jeune âge une chambre inférieure séparée du reste du cæeum par une véritable raliule.

11 ressort de là que cetto chambre inférieure prise par Hincks pour un corps spécial, loin d'être suspendue à l'estomac par un canal grêle et tendant à se rompre, lui est peut-être plus fortement unie qu'aucune autre partie, grâce à l'épaisseur des parois stomacales au point de jonetion.

Il est d'ailleurs complétement inexact que le détroit ì travers lequel passe la matière brune devienne progressirement plus long et plus étroit ; les figures ei-jointes ${ }^{1}$ représentent un même cæcum dessiné à la chambre elaire à quel ques minutes d'intervalle é prourent que l'aspect du canal change irrégulièrement et à tout moment suirant les contrations de l'estomac. Il m'est sourent même arrivé dans un Polypide en roie de résorption de distinguer encore au-dessous des bras flétris l'étranglement en question et de constater qu’il n'était ni plus prononcé ni plus étendu qu’à aueun autre moment de la rie du Polypide.

Si le mode de formation du corps brun décrit par Hincks ne peut être regardé comme correspondant aux fatits, comment done se passent les choses dans la Bicellaria ciliata?

Lì, comme partout ailkurs, le corps brun est le résidu du Polypide flétri et tout le Polypide sert à le former. Quant à la manière dont la transformation s'effectue, elle est en quelque sorte intermédiairo entre celle en usage chez les Bugules et celle des autres espèces : les tentacules commeneent par se flétrir et restent distinets pendant un ou deux jours, le cæecum où l'étranglement est encore visible se fond avec l'intestin, puis le tout se confond assez promptement en une mème masse.

Après avoir, je crois, mis hors de cause la théorie de Ilinclis et fait rentrer dans la loi commune un exemple que cet auteur me parait aroir faussement interprété, il ne me reste plus à discuter

${ }^{1} \mathrm{Pl}$. VIIl, fig. 8, $A, B, C$. 
qu'une opinion sur l'origine des corps bruns, e'est relle de Claparede, el je ne my apjesantirai pas longlemps, car elle est fondée sur un si grand nombre dobservations inexactes, qu'elle n'a trouvé que peu d'éeho patrmi les anteurs.

Glaparède ne croit pas ane le corps hrun puisse servir d'origine a de noureaux bolypiles, et la critique qu'il fait des opinions de Smitt est certainement très-judicieuse; mais quand il reut a son tour fornuler une opinion et déterminer le ròle de ce corps, il lait certinement fausse route. Pour lui le corps brun ne peut pas être le résidu du Polỵpide, paree qu'il a sur la résorption de celui-ci une théorie toute particulière.

D’aprìs lui le Polỵpide ne se flétrit pas, ne se désorganise pas, au terme de son existence, mais, pour disparaître, il passe en sens inrerse par toutes les phases qu'a pareourues le bourgeon pour se développer. Il rapetisse el diminue de rolume jusqu'à disparâitre, tout en conservant jusqu'au boul sa slructure el ses proportions.

Un tel fait, s'il était bien constaté, serait certainement très-surprenant et d'un haut intérèt pour la biologie; car je ne sache pas que chez aucun ètre arrivé au terme de sa vie le retour à l'enfance el à l'état embryonnaire soit la manière habiluclle de mourir. Il ne faut toutefois préjuger de rien, et si le fait est constaté il faudra bien l'adinettre.

Le malheur est que Claparède n'apporte à l'appui de cette surprenante théorie que des preuves tout à fait négatives et contestables. L'une des meillemres qu'il propose est ce fait qüil n’a jamais observé de Polypide flétri et en voie de désorganisation.

Aux extrémités des loranches, dit-il, tous les Polypides sont soit à l'état de bourgeons, soit adultes, tandis rue deux ou trois rangs plus bas ils sont tous disparus; il doit done s'en trouver dans l'intervalle en tráin de se flétrir, et ceux-là doirent présenter une apparence spéciale qui permette de les distinguer des bourgeons. Comme je n'ai jamais rien vu de semblable, je dois en conclure que les Polypides en roie de résorption ressemblent aux bourgeons et que pour cette ráison je n'ai pu les en distinguer.

A quoi tient que Claparède n'ait pas ru cet état intermédiaire de décrépitude par lequel doit passer el passe en effet tout Polypide arrivé au terme de sa croissance? Je ne le sais; toujours est-il que rien n'est plus fréquent, plus facile à obserier et mieux reconnu par tous les auteurs. 
S'appuyant sur sa théorie, le savant génerois concluait que puisque le Polypide, arrivé an terme de la métamorphose régressive, finissait par se réduire à néant, les corps bruns n’en pouvaient pas être les restes et devaient aroir une autre origine.

La coexistence souvent observée par lui dans une même loge d'un Polypide virant el d'un corps brun était encore un argument qu’il mettait en jeu, et celui-là arait plus de valeur.

Puisque, disait-il, il existe déjì un corps brun dans une loge oǹ se troure un Polypide en pleine vigneur, le premier ne peut évidemment pas être le résidu du second. Cette observation faite sur une Vésiculaire qu'il appelle à tort la Vallieria cuscuta n'a qu'me valeur apparente et tombe d'elle-même lorsqu'on sait, ce que nous savons déjà, que les Polypides se renouvellent sourent deux ou trois fois dans une même loge, et que dans les Vésiculaires leurs restes y demeurent relégués contre les parois, situation qui rend assez bien compte de l'opinion que Claparède adopte comme conclusion, et d'ailleurs sous certaines réserres, à savoir : que le corps brun est une sécrétion de l'endocyste.

Je ne pense pas qu'il y ait lieu d'insister davantage sur une théorie qui n’a d'ailleurs trouvé que peu de partisans; il reste donc bien établi pour nous que, dans tous les cas, le Polypide arrivé au terme de son existence se flétrit, se désorganise, se réduit en une masse globuleuse brune et que les corps bruns n'ont pas diatre origine.

\section{§ 2. Du rôle des corps bruns.}

Passons maintenant à l'examen de la seconde question : quelle est la destinée ultérieure du corps brun?

Peut-il servir d'origine à de nouveaux bourgeons ou de matière nutritive pour leur déreloppement?

D’après ce que je viens de dire de sa formation, on peut préroir ce que je pense de son rôle. Pour moi, le corps brun n'est qu'un résidu, un amas de matière inerte incapable de servir soit de matière nutritive, soit de matière plastique.

A l'appui de cette manière de voir, je présenterai plusieurs preuves; les unes seront tirées de la nature même de ces corps et de l'examen des matériaux qui les constituent; les autres ressortiront de la critique à laquelle je soumettrai les observations de mes adrersaires et 
par Iarpuelle j'espère démoutrer que le corps brun n’est pour rien dans le hourgeonnentent qu'on lui prète.

Prences tirees de la nature du corpss brun. - Le corps brun, nous renous de le voir, est un résiclu, le reste rle la maticre gui eonstituait un Polypide apres que celui-ci a subi la désorzanisation.

Peut-être cependant ce résidu est-il composé de matière plastipue et organisible pourant servir à un nouveau déreloppement.

Il n’en est rien; j’ai fréquemment et dans plusieurs espèces de Bugules el do Yésiculatires somuis des branches entières à l'éhullition prolongée dans la polasse. Après ce tratement, alors qu’il ne lestait dans tonte la colonie ancune pitilie molle rui ne fìt détruite, tous les l'olypides ayant disparu ansisi bien que l'endocyste et le système nerveux colonial, les corps bruns étaient encore reconnaissables et le plus sourent intacts. Les plus récemment formés avaient, il est vrai, perdu leur membrane enveloppe et par suite leur contour, mais les granules dont ils se composaient, répandus dans la loge et y formant un nuage jaune, étaient parfaitement reconnaissables. Ouant à ceur plus aggés qui vecupaient les vieilles loges de la base, leu1 membrane épaissie el sans doute chitinisée avait le plus sourent résisté si bien, que le eorps brun u’arait nullement perdu sa forme. A l'intérieur de celte membrane la polasse avait dù pénétrer comme elle pénètre dans l’intérienr des loges el des tiges; cependant le contenu n’était pas altéré.

Y a-t-il là en vérité les caractères d'un corps organisable et d'une matière plastique?

Voici maintenant des arguments d'un autre ordre.

Nitsche ${ }^{1}$ a produit à l'appui de l'opinion que je soutiens un fait que j’â rérifié et que je suis à même de confirmer.

Il a figuré un corps brun contenant une Diatomée et a sourent observé dans l’intérieur des fragments de test de Foraminifères et autres particules, restes des aliments dont l'animal se nourrit et qui se trouvaient dans son estonnac lorsqu'il a commencé à se flétrir. J'ai plusieurs fois pu conslater l'exactitude de ces faits et observer moimème des débìis de cette nature et je crois inutile d'en donner de noureaux dessins, mais je reproduis un corps brun de Bouerbankia imbricata dans lequel les plaques chitineuses fui rerêtaient le

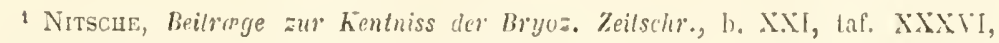
f. 10 . 
gésier à l'intérieur sont parfaitement conserrées et reconnaissahles ${ }^{1}$.

On remarifue sourent dans le rectum des Polypides en roie de développement un stylet byalin quils rejettent lors de la première défécation, quand ils sont devenus adultes el que Smitt a appelé le mécmim. Il n'est pas rare de tironver an fond des loges de petits annas de matière granuleuse incolore contenant un de ces corps; on peut être certain dans ce cas l'aroir affaire au reste d'un I'olypide qui s’est flétri arant d'aroir atteint son complet déreloppenent.

Au point de vue de son origine, e'est done un corps brun, bien qưil n’en présente pas la couleur, ce qui est dù, comme nous le verrons, à ce que les parois de l'estomae du Polypide n'araient pas eu le temps de contracter la teinte qui caractérise l'adulte.

En mème temps qu'elle fournit un nutrel appui à l'opinion que je soutiens sur l'origine du corps brun, la présence au milieu de sa substance, de ces corps étrangers, traces de l'alimentation ou restes de certaines parties le l'animal même, est certainement peu favorable à lat théorie qui veut en faire un corps reproducteur; mais pénétrons plus avant encore dans l'étude de la structure du corps brun.

De quoi se compose-t-il essentiellement, à quoi doil-il sa coloration si intense?

Quand un Polypide se flétrit arant d'aroir atteint l'état adulte, son résidu incolore finit par se réduire à un très-petit volume el même par disparaître tout à fait dans la substance du funicule, sous réserve cependant du stylet hyalin dont je viens de parler ou autres parties dures, telles que les plarjues du gésier qui, ne pourant ètre résorbées, restent inaltérées. Si, au contraire, le Polypide a vécu le temps ordinaire, s'il s'est nourri, si les parois de son estomac ont eu le temps de prendre celte coloration intense que l'on remarque chez les individus rigourenx, quand il vient à se flétrir il ne disparaît jamais entièrement et laisse toujours un résidu qui n’est autre qu'un corps brun bien formé qui s’entoure d'une membrane de plus en plus épaisse avec l’âge.

De quoi done se compose ce corps brun? De tout ce que le Polypide adulte et vigoureux arait de plus que le bourgeon, c'est-ì-dire de particules alimentaires contenues dans l'estomac et par-dessus tont de ces granulations brunes qui surchargeaient les parois de cet organe. Or, que sont ces granulations? Sont-ce de jeunes cellules 
hépatigurs, comme le veut Smill? Pour le savoir, suivons le déreloppement d’un hourgeon à l'extrémité d'une branche. lons parois de l'estonat sont d'abom incolores, puis, un pen plus tard, et dans certaines cipiees, arant mème que le bourgeon se soit eneore épanoui, elles présentont quelenes fines pipuetures brunes. Si l'on examme alors les parois de cet nrgane sour wn fort grosissement et après

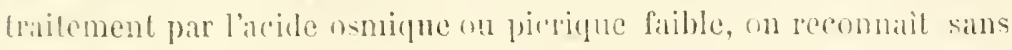
poine que ees ponctuations ne sont antres que les rontenus de eellules polygonales qu’i bon droit on pent nommer hípatiques. Si l'on suit leur dévelopiement daus lat Buyult flubellata, où elles apparaissent de bonne henre, et surtout dans les grandes espèces de Pédieellines où elles sont d'ailleurs absolument les mêmes que dans les aulres hryozoaires, on roit que leur contenu brum augmente peu it peu de rolume jusqu’a leur maturité; clles se détachent alors it la manière des cellules épithéliales, crèrent, et rersent leur contenu dans la carité de l'estomar. Ge contenu n'est autre du'non gramule brum, sans donte environné d'un peu de liquide. Les grannles sont remuós avee les aliments pir l'atetion des cils vibratiles le l'estumac el du recum, dans line duquel ils tournent longtemps d'un mourement irès-régulier ${ }^{1}$.

Loistrue le Polypide se flétrit, son estomac contient done m grand nombre de cellules hépatiques en place sur les parois cn mème temps que des granules sortis des cellules désagrégées. Ciest à l'ensemble de ces cellules et de ces graniles quest due la coloration des copps bruns, car la plus grande partie de lecir substance en est composée.

On roil par lik que cette substance, loin d'être constilnće par de jennes cellules prètes à revirre, n’est formée que par des produits de sécrétion ou d'excrélion, comme on roudra l'entendre.

Ainsi, pour nous, par son origine et par sa nature, le corps brun est incapable de seprir au développemen!.

l'ajouterai maintenant que, dans aucun cas, je ne l'ai vu servir à cet usage, bien que je me sois placé rlans les mêmes conditions que les auteurs qui en ont déerit le hourgeonnement.

Voici une loge de Borcerbantia imbricata ${ }^{2}$ dans laquelle le bourgeon se déreloppe sur les parois à l'extrémité du funicule et fort

1 Pl. VIH. fig. 11, et pl. IX, fig. 11 et 12.

2 P. VI, fig. 3. 
loin du corps brun préexistant. En voici une autre de Vallevia cuscuta, où un polypide naissant à côté d'un corps brun, mais nullement à ses dépens, poursuit tout le cours de son déreloppement, sans que celui-ci soit altéré en aucune façon ${ }^{1}$.

J'ai observé un très-grand nombre d'échantillons de Bowerbankia imbricata, jai suivi le développenent de plusieurs branches d'une manière continue pendaut plusieurs mois, et je n'ai jamais ru le corps brun participer en rien au bourgeomnement.

A mesure qu'il s'en forme un nouveau, il est relégué dans le fond de la loge contre les parois de l'endocyste jusqua ce que la loge venant à mourir elle-même, il tombe arec elle ou bien en sorte par le col.

Voici un autre fait dont on ne semble pas suffisamment tenir compte.

Une loge ne produit pas un nombre indéfini de Polypides et tout le monde sait que la base des Bugules et des Eschares est composéc de cellules mortes; le demier venu des Polypides qui se succèdent dans une loge produit, comme ceux qui l'ont précédé, un corps brun qui ne doit pas être d'une autre nature que les premiers. Celui-là, cependant, ne bourgeonne jamais: at quoi cela tient-il? et comment ne tombe-t-il pas promptement en décomposition?

11 finit, il est rrai, arec le temps par s'altérer, mais nullement comme le ferait un corps organisé ou composé de matière organisable; il change de couleur, passe au jaune sale, puis au vert, mais sans que son contour soit de longtemps elfité et il est encore reconnaissable alors que les branches du T'olypier tombent en mor'eatux, comme il arrive pendant thiver pour certines espèces.

Ce sont là, me dira-t-on, des preures négatives el qui noont pas de valeur en face des observations précises de Lovén, de Smilt, de Hincks, de Repiachoff. Anssi, je ne m'y arrêterai pas plus longtemps et j'aborde immédiatement l'examen et la eritique de ces auteurs.

Lorén ${ }^{2}$, eité par Smitl, dit aroir observé lis transformation des corps bruns en larves ciliées dans la Vallieria cuscuta.

J’ai moi-même observé cette espèce pendant longtenups, j’ai ru les corps bruns, j’ai vu les larves et de plus les œufs d'où elles provien nent el qui n'ont rien de commun aree les corp.s bruns. Ceux-ci, je

1 Pl. VII, fig. 9 et 10.

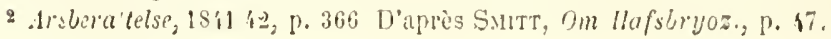


puis l'affirmer, se forment, comme partout aillenrs, aux dépens du Polỵpide en voie de résorption.

Quant a l'reuf, il est risible dans la loge pendant la vie du Polypide et bien avant la formation du corps lorm.

11 se présente d'abort comme un petit amas de protoplasma clair primitivement atcompagné d'un second, ear les cuf's naissent par paires; puis il prend une teinte janne qui derient plus foncée à mesure qu'il grandit el c’estévileument dans cet ćtat que Lovén l’a pris pour un corps Jirun.

I: en diffère, eepentant, par sa forme régulièrement arrondie aussi bien que par son contenu finement granuleux et non grumeleux, comme celui des réritables corps bruns.

Les observations de Snift et de Hineks peurent paraître au premier aborl plus embarrassantes. Smitt ${ }^{1}$ lécrit le déreloppement de Polypicles sur le corps brun mème et aceompagne sa deseription de figures malheureusement trop petites pour qu'on puisse bien juger de la prosition des parties.

Quant à llincks, il s'exprime dans les termes suivants :

"Sur la surface supérieure du corps brun apparaît une saillie de matière grise granuleuse qui est l'origine du bourgeon; ce bourgeon se dércloppe en un polypide dans les parois stomacales duquel se fond le corps brun qui lui a donné naissance. Les Polypides nés de cette sorte ont les parois brunes, tandis que les autres sont incolores, n et il appruie cette deseription d'une figure qui ne laisse subsister aucun doute.

Si done jai constaté que, chez deux espèces de Vésiculaires, les bourgeons se forment tout à fait indépendamment des corps bruns, le plus souvent loin d'eux, je dois convenir qu'il ne m'est plus possible, en face des observations précitées, de faire, de ce rôle passif du corps brun, une loi générale. S’il est vrai que, dans de nombreuses uspèces, le bourgeon naît du corps brun mème el finit par s'incorporer sa substance, comment ne pas le considérer comme un véritable corps reproducteur, et admettre qu'il est apte à bourgeonner?

Les observations récenles et très-prícises de Repiachoff ont jeté un nowreau jour sur celle ruestion en complétant celles de Smitt et

1 Om llafsbryoz. Utveckl. och Feltkrop., [1. V, fig. 17-19. 
de Hincks et en nous faisant connaître daus le détail ce que ces deux auteurs n’ont fait (p'ébaucher : les véritables rapports du corps brun et du bourgeon; elles nous mettront peut-ètre en bon chembr.

Pour Repiachofl, il n'y a pas de relation d'origine entre le colpss brun et le bourgeon; celui-ci peut naître directement sur le corps brun aussi bien qu'à une certaine distance. Nais, tôt ou tard, il finit par s'en rapprocher el par l’engloher tlans la cavité naissante de son estomac. Comment se fait cet enveloppement? Repiachoff ne le prérise pas; il faut bien, dit-il, qu'il y ait perforation des parois de l'intestin et il est probable que cette perforation se pratique dans le roisinage des tentacules maissants qui se résorbent pour repousser ensuite.

Le corps brun entre alors dans lintérieur du Polypide accompagné de quelques granules qui sont le résidu de ces tentacules flétris. Les granules servent de nourriture au Polypide, tandis que le reste passe dans le rectum pour ètre probablement éracué par la suite. L'autcur russe termine enfin par une conclusion tout à fait inattendue, en assimilant le corps brun à une sorte de vitellus.

Ces observations sont accompagnées de planches dessinćes d'après la Tendra zostericola et une lepralia indéterminée et bien faites pour inspirer la conviction, car elles sont d'un réalisme poussé parfois jusquì̀ l'extrème.

Je suis à même de confirmer ces données et de les compléter par les observations suriantes:

La figure 1 de la planche IX représente un bourgeon assez arancé d'Encratea chelata, dessiné le 1 r juin 1877 ; l'extrémité inférieure de son crecum stomacal est en ruelque sorte confondue arec un corps hrun. Celui-ci est dès à présent entouré de ces granulations jaunitres que Repiachnff a pris pour le résidu de tentacules naissants el qui l'environnent souvent au milieu de la loge bien arant qu'aucun bourgeon se roie dans le roisinage ${ }^{1}$.

Le lendemain, le corps brun a pénétré dans l’intérieur de l’estomac, non pats dans le roisinage des tentacules, mais par le fond du eul-de-sac dont les parois se sont refermées derrière lui, emprisonnant rquelques-mus des granules qui l'atcompagnaient et qui restent comme les témoins de son passage?

l.e 3 juin, forecant l'entréc de lintestin, le corps brun, eneore en-

1 I'. Vlll, fig. 12.

2 P. IX, fig. 2. 
veloppé de sa membrane, est passé dans le rectum refoulant devant lui le méconium quis'y trouvail trejà".

L'estomar n’arait conservé d'aulres pestes du corps brun que les quelques gramules qui adhéraient ì ses parois.

Je u'ai pu suirre plus loin le sort du corps brun sur le mème sujel, le bolypide s'élant acoidentellement flétri; mais èn roici un antre qui élait, le 7 juin, absolmment dans les mômes conditions gue le précédent le 3. Son rectum ítait distendu par un eorps brun aceompagné d'un stylet hyalin. Le 8, le P’olypile etait parfaitement virant; mats il n'y arat plus dans son intestin aucune lrace de l'un ni de l'autre, si ce n'est quelques lógères granulations brunes.

Evidenment, le corps brun, encore enveloppé la veille de sa membrane résistante, n’avil pas été absorbé par les parois du rectum, de toutes les parties du lube digestif celles qui sont le moins propres à l'absorption ; il arait sams aucun doute été rejeté pendant la nuit à tratrers lanus en même temps que le méconium.

Cette observation, jointe it celles de Repiacholl, nous rend compte juspu'a un certain point des faits dont Smitt et Hincks ont tiré des conclusions trop hatives, mais elle nexplique pas encore conment ce dernier autenr a pu roir le corps brun se fondre dans les parois du lube digestif du Polypide et les rendre distinctes par leur couleur de celles des Polypides nés de lendocyste. Toici quelques figures dessincés dapres la Lepralia granifera qui seront peut-etre plus démonstratives ${ }^{2}$.

La manière dont l'estomac finit par englober le corps brun est tout à fait la mène que dans l'Eucraten chelata.

Le tissu encore trèsplastique dont sont constitués à cette époque les parois de lintestin, s'étend sur la surface du corps brun de manière à l'envelopper daus une sorte de poche: les parois internes de celle poche, qui no sont atules que celles mèmes du eacum stomacal, s'amincissent, se perforent; le corps brun passe par cette onrerture dams l'estonac; les parois externes de la poche reviemuent sur elles-mèmes et servent à boucher l'onverture, dont il no reste bientot plus de trace visible.

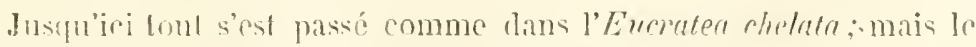

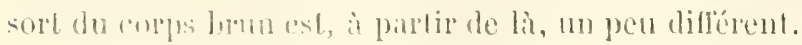

1 . Jí ché témoin de ce passage, qui se fait par une contraction brusque du cxemm coìncidant avec une dilatation énorme du pyiore.

\& Pl. IX, fig. $3,6,7$ of 8 . 
Dans l'espèce qui nous occupe, en effet, son enreloppe, très-mince, ne résiste pas à l'action des sucs de l'estomac, non plus qu'au mouvement imprimé par les cils vibratiles et aux contractions des parois intestinales; elle se détruit, et les granules bruns qu’elle contient, mis en liberté, se répandent dans l'estomac, y tournoient et sont ensuite promptement éracués après avoir passé dans le rectum.

Pendant leur séjour dans cet organe et dans l'estomac, les granules leur communiquent leur teinte, et c'est évidemment là l'origine de cette opinion de Hincks, que les Polypides qui naissent des corps bruns sont seuls colorés.

- En réalité, cette coloration, qu'on peut dire arlificielle, est bientôt remplacée par la coloration naturelle, qui résulte, comme nous l’avons ru plus hant, du développement de cellules hépatiques qui se fait dans les parois intestinales de tous les Polypirles, quelle que soit leur origine.

Si maintenant on objecte que cette désagrégation du corps brun et cette diffusion de sa substance dans l'estomac ressemblent à une véritable digestion, il sera légitime de répondre que ce n'est qu'une apparence, puisque les granules sont rejetés peu à peu et intacts par l'anus. Je ne reux pas affirmer qüil n'existe encore entre leurs interstices quelques partics assimilables; mais je ne puis pas pour cela considérer le corps brun comme destiné spécialement à la nutrition du bourgeon, puistue dans l'autres espèces, comme l'Eucratea chelata, je le rois sortir intaet du rectum. Il me semble bien plus croyable que celte désagrégalion n’est qu’un mole d'évacuation rendu nécessaire par la trop grande dimension du corps brun, ef facile par la délicatesse de son enveloppe.

\section{CONCLUSIONS.}

De loutes res observations, quelles conclusions devons-nous tirer?

Tous admettons que, bien que, chez certaines espèces (Vésicularres et autres), les corps bruns soient, à mesure qu'ils se produisent, relégués en guelque point de la loge, il en est d'aulres chez lestuelles de nouveaux bourgeons ne naisent pas sans délarrasser la zoćcie des

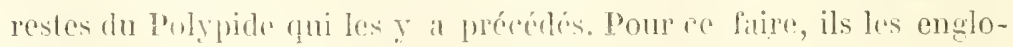
bent dams la cavilć de lemr lube digestif et les rejettent par l'anus, soit en enticr, soit après les aroir désagrégés.

letle manicre de roir, appuyée sur les obserrations que je viens 
dexposer, me parait rendre compte des faits pour le moins aussi bien que celle de Smitt et de Hincks; elle a sul elle l'arantage de tenir compte des observations de Repiachoff, qui n’étaient pas encore liates a l'époque où ont écrit ces denx autens: enfin, elle est en accort eomplet aree l'opinion de Nitsche, et véritablement avee tous les laits yui obligent à ne voir dians le corps brun yutun résidu, un amas le matière inerte.

(luant à ce qui est de Ia manière dont le bourgeon se déreIoppe à la surface du corps hrun, comme Smilt et Hincks, nous reconnaissons que le bourgeon peut naîle sur la surface de ce corps tans de nombreuses espèces; mais fant-il conclure de là que cest aux dépens de sa substance mème qu’il se déreloppe? Nons ne le croyons pas.

l'esons bien les paroles de l'auteur anglais : "Sur la surface supérienre du corps brun, dit-il, apparait une saillie de matière grise granuleuse, qui est l'origine du bourgeon. »

Remarquons d'abord que le corps brun, quand il bourgeonne, est toujours suspendu à l'une des mailles, et ordinairement au cordon principal du réseau qu'on nomme systeme nerveux colonial, el sur la nature duquel j’aurai à m'étendre tout à l'heure ${ }^{1}$.

La matière de ce réseau, non-sculement le retient par un ou deux points, mais l'enveloppe dans une couche protoplasmique, ordinairement fort mince, anx dépens de larguelle, soit dit en passant, se constitue l'enveloppe du corps brun. Il n'est certainement pas toujours facile de décider si c’est aux dépens du corps brun lui-même ou aux dépens de cette couche protoplasmique que se forme cette "silllie de matière grannlense", qui est l’nrigine du’bourgeon. Cependant, si on l'examine de près, on verra que celte saillie est toujours séparée du corps brun proprement dit par la membrane enreIoppe de celui-ci; et cela doit ètre, puisque, dans l'Eucrater chelatu, nons avons ru que le corps hrun arait couservé celte membrane intacte jusque dans l'infórieur même du lube digestif.

Xous arons par là la prenve directe que ce n’est pas aux dépens de la substance inerte du corps brun que le bourgeon se développe, mais bien aux dépens de la conche de protoplasne qui l'envirome et applartient atu système nerreux colonial.

Cette origine, qui me semble démontrée par les faits, pourra pa-

t Voir pl. Xill, fig. 1. 
raître suspecte au premier abord, ì cause du nom nème qu'on dome à ce tissu depuis Fritz Müller. Ces doutes dispatraîtront, jespère, lor'squ'on saura que le système nerveux colonial, auquel je ne conserve ici que provisoirement le nom sous lequel on le comnait, est un tissu essentiellement apte aux fonctions reprorluctrices, el que les bourgeons se développent toujour's dans sa dépendance.

En dímontrant linerie du corps brun, nous faisons rentrer dans un cas déjì connu le déreloppenent par germ-capsules Igroddliapselbildning), admis par smilt et Hincks. Nous diminuons d'iutant le nombre des origines qu’on domait aux bourgeons des Bryozoaires, nombre que j'espère dans un prochain chapitre lamene: à l'unité.

Pour teminer ce débat, je formulerai sur la nature, le róle et l'origine des corps bruns les conclusions suirantes:

$1^{\circ}$ Tout Polypide adulte arrivé au terme de son existence, loin de subir une métamorphose régressive, qui le ferait repasser successivement par tous les états antérieur's, se llétrit, se désorganise et se réduit intégralement en une masse arrondie de matière brune, qui est connue sous le nom de corps brun;

20 Le corpsbrun, résidu d'un Polypide antérieur, se compose principalement des granulations brunes enntenues dans les cellules hépatiques de ce dernier, auxquelles s'ajoutent parfois des débris des particules qui ont serri it son alimentation, ef les parties dures qui entraient dans sa constitution comme dents et plaquer du gésier. Le tout est enfermé dans une membrime qui s'épaissit avec l'age, et qui est produite par la couche de protoplasme qui l'environne;

$3^{\circ}$ Le corps brun, composé de matière inerte, est incapable de servir, soit de point de départ à de nouveaux Polypides, sont te matière nutritive pour leur développement, et les humrgenns qui peurent se monirer it sa surface naisient de la conche de protoplasme qui l'enviroune et qui dépend du systeme nerveux colonial, antquel il est suspendu;

$4^{\prime}$ Dans certaines espèces, les noureaux bourgeons qui naissent dans la loge n'entrent jamais en relation arec les corps hruns, qui peuvent se irouver au nombre de deux, ou mème trois, dans une zoécie hahilée par un Polypide.

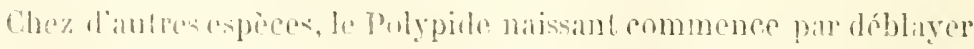
sa loge du corps hrun qui peut s'y trouver. A cet effel, qu'il se soit déreloppé ì quelejue distance du corps brun ou sur sa surlace mêne, il finit tòt su tard par entrer en relation aver lui. 
11 l'englobe dans sa cavité stomacale, après quoi il le rejette au dehors par' l'anns, soit en entier avee le méconiun, soit par pareelles après l'atroir désagrégé.

\section{CHAMTRE II.}

DU hOLE ET DE L.I NATLRE DU SYSTEME NERYEUX COLONII, DES BRYO\%OARES.

\section{\$1. Du systeme nerveux colonial de lu bourerluntiu imbricata Johnst.}

Iien que j’aic, dès le débul de mes recherehes, examiné le syostème nerveux colonial dans un certain nombre de groupes différents, afin d'citre bien fixé sur ce que les auteur's désignent sous ce nom, j’ai bieulòt senti la nécessité de porter mon attention plus spécialcment sur un type bien choisi, afin de pouvoir, en concentrant mes efiots sur un seul point, arriver plus promptement à en posséder une eonmaissance approfondie, el d'ètre a mène ensuite de le prendre pour terme de comparaison.

Je me suis adressé tout d'abord à la Burerbankin imbricata.

Son abondance à hoseoff, sa transparence et les autres arantiges qu'elle offre à l'obserrateur, et flont j’ai déja parlé, u’ont pas seuls intluencé mon choix; un autre motif plus sérieux m’a surtout engigé ì prendre ce type comme le prenier objet de mes recherehes.

C'est en effet dans une Yésiculaire ou da moins dans un genre très-voisin, la Serialaria Coutindrii, Mïller (si celte espèce, toutefois, peut être bien légitimement déterminéc comme sérialaire), grue Fritz Niuller a déerit, pour la première fois, sous le nom de systime nereud culonint, un cordon transparent qui pareourt lis lige, renllé en ganglions granuleux au nirean des neuds, et accompagné d'un plexus plus on moins riche '.

Ce ménoire du sarant allemand a servi de point de dípirt anx anteurs éminents yai ont trouvé l'analogaue de ce syiteme dans les antres Brynoaires marins, et notamment dans les Cheilnstomes, où il est souvent plus difficile à voir.

Smitt, Claparòde, Hinckis el la plupart des observaleurs, ont été d'accord non-seutenent pour reconnatre lexistence de ce sistime

1 Fritz Mulder, Archiv fur Nalurgeschichte, 26 e Jahtgang, 1860, p. 310-318, pl. XIII. 
chez les Bryozoaires marins, mais pour affirmer ou admettre sa nature nerveuse, el, si je mets à part Reichert, qui a émis à ce sujet une opinion toute partieulière ${ }^{1}$, je ne commais guère que le docteur 11. Nitsehe qui, tout en le décrivant favee une grande exactitude au point de rue anatomique, ne se prononee pas sur sa véritable nalure, mais se refuse tout au moins à le considérer comme un système nerveux ${ }^{2}$.

Je ne m'attacherai pas a disenter les excellents arguments qu'il inroque à l'appui de sa thèse, me réservant d'y revenir plus tard; mais, puisque les auteurs les plus autorisés sont partagés d'opinion sur ce sujet, il me semble naturel de remonter au mémoire qui a servi de fondement à la théorie du système nerrenx colonial, affin de voir, en les soumettant au eontròle de nouvelles observations, si les faits qui y sont exposés sont bien de nalure à légitimer les conclusions qu’on en a tirées.

Fritz Müller a reconnn dans la tige de la Vésienlaire qui fait l'objel de son étude :

$1^{\circ}$ Un eordon central, qui, pareourant dans sa longueur chacun des articles de la tige, se divise à son sommet en aulant de branches que eet article iomrnit de ramifications ${ }^{3}$;

20 Des ganglions granuleux, qui se trourent à la base de ces branches, aussi bien qu'à la base des zoécies ${ }^{4}$;

$3^{\circ}$ Un plexus, qui est superposé au cordon central et qui relie entre eux les ganglions des branches et ceux des zoécies;

$4^{\circ}$ Un nerf', qui se rend du ganglion basilaire des zoécies au bourgeon ${ }^{3}$, et pent-ître aussi à l'intestin du Polypide adulte.

Ces diverses parties sont aussi nettes, aussi faciles à observer dans la Bourerbankiu imbricata qu'elles peuvent l'être dans la Serialaria Coutinhii. Yoyons done si un examen attentif de leur structure et de leurs fonetions nous permettra de les eonsidérer, avee Fritz Müller, comme étant de nature nerveuse.

A. Fnnicule. - Prenons d'albord ce nerf qu’il a cru voir (einige

1 ReICIERT, Vergleichende anatomische Untersuchungen uber Zoobotryon pellucidus (Alk. Akad. Berl., 1869 ).

2 1)r II. Nitscile, Reitroge zur Kenthiss der Bryozoen (Zeitschriftf. wissens. Zool., Band XYI, viertes eft, 1. 434-435, et Quart. Journ. of micr. sc., t. XI, 1871, p. 153).

3 PI. YI, fig. 1, cn.

" Pl. VI, fig. 1, g.

5 Pl. VI, fig. 2, $n$. 
Mal gesehen zu haben Glaube) se rendre du ganglion hasilaire de la

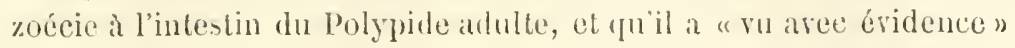
s'attacher au bourgeon.

Ce condon existe manifestement chez l'adulte anssi bien que dans le bourgeon ; il ratlache l'm et l'antre au ganglion basilitire el, par suite, au reste dus systeme.

T'antòt granuleux, tantòt transparent, et alors fort diflieile à roir, il n'est reconnaissable, chez l'adulte, que quand l'animal est bien épanoui.

Il ressemble alors à une amarre tendue qui rattache l'estomac an fond de la loge, tundis que le lophophore est retenu lui-même par les muscles grands rétracteurs.

Son diamètre est au moins égal au tiers de la largeur de l'estomac. Pour un filet nerveux, c'est là, il faut en convenir, une assez belle taille.

Fritz Müller dit n'avoir pas pu constater ses rapports arec le ganglion œsophagien. Cela ne doit pas étonner, puisqu'il n’est pas même parrenu à s'assurer positivement de son existence.

C'est à l'extrémité du cacum de l'estomac que ce cordon vient s'attacher, et il se prolonge même, en s'atténuant peu à peu, à une certaine distance, sur la face ventrale de cet organe 1.

Il est extrêmement extensible et contractile, et contribue certainement par cette dernière propriété à faire rentrer le Polỵpide dans sa loge, agissant alors, en quelque sorte, comme un troisième musele rétracteur.

(luand un Polypide se flétrit el meurt, le funicule, carr e'est ainsi que je l'appellerai maintenant, reste en connexion avec lui, jusqu’à ce que, transformé en un corps brun, le zö̈de aille se ranger sur les parois de la loge, pour faire place à un nouvel individu en roiu de développement.

Mais pendant cet intervalle il change fréquemment die forme et d'aspect, el, par l'intermédiaire des tissus désorganisés de l’ancien habitant de la loge, il contracte souvent arec l'endocyste des adhérences qui, sous forme de tractus, l'attachent, comme par des amarres, en différents points de cette membrane et l'empêchent de s'affaisser.

Lorsqu'un bourgeon se forme à nouveau sur l'endocyste d'une loge 
déjà ancienne ${ }^{1}$, on le voit généralement de très-bonne henre pourvu d'un funieule qui atteint alore presque son diamètre; et depuis mème que mon attention a été portée sur ee point, je n’ai jamais ru se former dans ces conditions aucun bourgeon qui manquat de cette atfache. Je suis done porté à croire que les bourgeons se développent de préférence sur les points de l'endocyste, où se sont fixées les amarres dont j’ai parlé tout à l'henre, ct rque e'est ainsi que, dès leur plus jeune ige, ils se trourent naturellement pourvus d'un funicule?

Si maintenant on reut prendre en considération : l'énorme diamètre du funicule par rapport à l'estomac de l'adulte et par rapport au bourgeon; la large suriace par laquelle il s'attache à l'un ou à l'autre; ses propriétés contractiles; les changements de forme ru’il peut subir dans l'interrègne de deux Polypides; si fon tient compte de sa strueture histologique qui nous montre dians l'adulte des cellules fusifornes, très-semblables à celles que j'ai reconnues dans le cordon central, et qui ne paraissent avoir rien de commun arec les ćléments d'un tissu nerreux ${ }^{3}$; si enfin on se rappelle que cest dans le tissu mème du funicule que, chez certaines espèces, le testicule se dércloppe comme Van Beneden l'a décrit dans le genre Laguncula, il me semble qu'il sera difficile de continuer à le regarder comme un simple nerf se rendant du ganglion basilaire de la loge à l'estomac, et ayant arec le ganglion osophagien des rapports qu'on suppose exister, mais qu'en somme on 11 'a jamais vus.

B. Ganglions. - Passons maintenant à l'étude des "ganglions" qui se trouvent à la base des zoécies et des articles de la tige.

Il suffit de jeter les yeux sur les figures qui accompagnent le mémoire de Fritz Büller et dont je reproduis l'une sur mes planches", pour s'assurer que cet auteur ne s’est pas fait une idće exacte de leur constitution, pas phus que des communications que les zoécies ont avec la tige ou rque les articles de celle-ci ont entre eux.

Toutes ses figures, en effet, représentent les trones nerveux comme passant librement el sans obstacle d'un article à l'autre, et se renflant

4 Pl. VI, fig. $3 f$.

2 Cette induction, formée en mars 1577, a été complétement confirmée, comme on le verra par la suite.

3 Pl. VI, fig. $5 f$.

4 Pl, VI, fig. 1. 
cu annglions an nivean des articulations. C’est en rain yue dans ces mèmes planches on chereherait les choisons thansoresales qui se trouvent toujours à ce nivean sépanant les loges les unes des antres et compant par le milien ees prétendus ganglions ${ }^{1}$.

11 cst évident yu'en desimant ses figrures l'antenr n'a pas en connaissane de ces diaphragrmes, il le dit d'atlleurs lui-mème datus une sorte de post-scriptum interralé entre pancuthises, p. 313, et dans lequel il recomnaît, après aroir cxaminé d'autres espèces de Clónostomes, lexistence de sépanalions entre les articles de la tige ${ }^{2}$.

Celte constatation tardive, et dont Reichert a relevé depuis l'insuffisanee, reste d'ailleurs sans inlluence sur la description qu'il latit des gangrlions et sur les conelusions de son mémoine quelle aurait dù cependant modifier profondément, ainsi que nous allons le voir.

Comme Reichert l'a décrit, en effet, dans le Zoobotryon pellucielus ${ }^{3}$, et comme je l'ai recommu de mon coté dans la Bouerbankia imbricata, qu’on les considère, daus la tige ou sur ses côtés, comme articles du tronc ou comme zocicies, ćléments qui sont d'aillenrs équivalents au point de vue morphologique, denx loges alultes sont toujours sćparées l'une de l'autre par un diaphragme, cloison chitineuse comme l'ectocyste, percéc en son centre d'une perforalion très-fine * dont le diamètre n'atteint guère plus d'un centième de millimètre et reste toujours inférieur à celui du trone nerreux et a plus forte raison du ganglion.

Sur chacune des faces de ce disque, le cordon central de la loge correspondante s'épate. en se confondant avec l'endocyste et forme ainsi mn amas de matière granuleuse ${ }^{5}$.

La juxtaposition de ces deux amas, qui ne sont séparés que par une cloison souvent fort mince, présente assez bien l'apparence d'un simple renflement du cordon central qui se continuerait sans interruption d'une loge dans l'autre.

1 Pl. VHI, fig. 1.

2 a Spæterer Zusatz: Nach Beobachtungen an anderen Ctenostomen Bryozoen vermuthe ich dass die enizelnen Glieder durch eine von der Ilülle ausgehende Quere Scheidewand getrennt siud. "

3 ReICHert, Vergleichende anatomische L'ntersuchungen über Zoobotryon pellucidus (Ath. Akad. Berl., 1869).

4 Les diaphragmes du Zoohotryon pellucidus sout percés d'un trou environné de plusieurs autres, ce qui constitue ce que Reichert appelle la Rosettenplatte, dans la Bowerbankia imbricala, il n'y a qu'une seulc perforation.

b. Pl. Vll, fig. 1 et $3 \mathrm{~g}$. 
Le prétendu ganglion est donc formé de deux moitiés bien distinctes, séparées par une cloison cl n'ayant entre elles d'autre communication qu'mue perforation très-fine qui la traverse.

Il y a loin de là à la structure que représentent les figures de Fritz Müller; il y a loin de là aussi à ce qu'on entend généralement par un ganglion.

C. Cordon central et plexus. - Reste à étudier la partie essentielle et fondamentale du système nerveux colonial, c'est-à-dire le cordon central avec le plexus qui en dépend ct n’en diffère pas l’ailleurs pour la structure.

C'est en vain que I'on ehereherait dans le mémoire de Fritz Nüller des données histologiques propres à légitimer la qualilé de lissu nerreux qu'il prête à ce cordon. L'auteur n'a pas cherché à se rendre compte de sa structure intime, et l'apparence extérieure du lrone, du plexus et des ganglions, jointe à des raisons physiologiques que je discuterai en dernier lien, sont les seuls arguments sur lesquels il se fonde pour en faire un système nerreux.

Quclque obscurs et peu définis que soient les caraclères histologiques des centres et des cordons nerveux dans les animaux inférieurs, la forme des éléments qui le composent est cependant bien un argument dont il faut tenir compte quand il s'agit de déterminer la nature d'un tissu.

Histologie du cordon central. - Si l'on examine sur le vivant le cordon central, on y reconnait bien, lorsqu'il est très-transparent, quelques striations longitudinales; on le voit très-souvent dans certaines parties de la tige, chargé de granulations réfringentes plus ou moins abondantes et parlois si serrées, qu'elles le masquent presque complétement et ne trahissent que sa direction".

Ce dernier fait m'avait, je l’aroue, à lui seul, déjà fortement incliné à douter de la nature nerveuse du cordon ${ }^{2}$, car je ne sache pas qu'un tissu nerveux ait pour fonction ordinaire de servir de réserve aux matières nutrilises ou plastiques. Mais en somme, par l'examen des objets frais, on n'obtient aucune notion précise sur la structure intime de ces parties.

1 Pl. VII, fig. $3 \mathrm{cp}$.

2 Ces doutes, fondés sur les mêmes faits, ont été exprimés en 1860 par le professeur Giglioli, qui reconnait que " le anastomosi sono fili di granuli ben differenti nell" aspetto da veri elementi nervosi " et ne pent se rallier à l'opinion de Fritz Muller: "Che dotara animali eosi semplici di un sisteme nervoso eosi complicato. „ (Atti delle R. Accad. di Torino, 1866, p. 131.) 
Il nen cst pas de mème si on les traite par un réactif acide.

L'acide chromique, l’acide pierique rénssissent à la longue, mais c'est l'acide chlorhyglrique faible et l'acide osmique très-étendu qui m’ont toujours fourni le plus promptement les meilleurs résultats.

Si l'on a affare à unc branche où le cordon central soil bien transpareut, on roil immédialement s'aceuser arec la plus grande netteté des cellules fusiformes accolécs, assez allongées, pointues aux deux extrémités, plus on moins rentlées au milien, dirigées toutes dans le sens longitudinal ${ }^{2}$ et qui ne ressomblent en rien aux prétendus canaux dont se composerait le cordon central d’après Reichert. Elles sont finement granuleuses, présentent ordinairenent quelques granmles plus gros et très-rélringents, mats je ny ai jamais ru de noyaux à nucléoles aussi nets que ceux que Nitsche et Claparède ont figuré dans des partics analogues chez la Flustra menbranacea ${ }^{2}$ et chez la Scrupocellaria scruposa ${ }^{3}$.

Ces deux auteurs se sont en eflet oceupés de l'histologie du sỵstème nerreux des Cheilostomes.

Ils ont cxaminé, le premier un fragment du funicule funicular platte), le second une porlion d'un filet pris dans une loge.

'Tous deux ont reconnu dans ces parties des cellules fusiformes fort analogues à celles que je préscnte ici comme appartenant à la Bowerbankia, sauf en ce point que les noyaux sont beaucoup micux accusés chez ces Cheilostomes.

Si ces deux autenrs s'accordent à peu près sur les faits, ils ne s'entendent pas aussi bien sur leur interprétation.

Tandis, en effel, que Nilsche ne roit rien de nerveux dans ce tissu, Claparide regrette de ne pouvoir pas décider " si ces cellules fusiformes dont les extrémités senublent s'effacer se continuent par une seule fibre ou par un faiscean de fibres". ")

Les planches qui accompagnent ce travail ${ }^{5}$ seront beacoup plus démonstratives (qu’aucune explication; dans le cordon je n’ai janıais vu les prolongements que Claparède suppose à ces cellules et si des

1 Pl. VIl, fig. 3.

- Nitsche, Lurr die Analomie and Enlwiclichngsgeschichle von Flustra membranacea Zeilschrift für wssens. \%ool, Band XX1, Laf XXXYII, fig. [บ).

3 Claparèe, Beitrage zur Anatomie und Entuciclehngsgeschichte von der Seebryosoen (Zeitschrift fü wissens. \%ool., Band XXI, taf. IX, $1 \mathrm{G}$ ).

+ Zeilschrift für wissens. Zool., Band XXI, p. 159.

s Pl. VI, fig. 8 . 
cellules détachées s'atténuent à l'une de leurs extrémités ou sont relićes entre elles par des filaments sareodiques, elles ne ressemblent pas pour cela à des eellules polaires. Je ne rois done pas bien à quelle catégorie d'éléments nerveux il serait possible de les rapporter, je dois même ajouter qu'elles présentent ecrlains caractères et subissent certaines modifications qui paraissent incompatibles avec la nature d'un tel lissn.

Par leur forme, en effet, et par lour contenu, elles different les unes des autres, non-seulement dans deux branches voisines, mais dans la même branche, en deux régions d'un mème article et sur les deux bords d'un mème cordon.

J'ai dit, en effet, tout ì l'heure rue le cordon central, transparent en certains endroits, était, en d'autres, si fortement chargé de granulations ' que sa strueture et mème sa forme en étaient complétement masquécs. Nème dans les parties les plus transparentes, l'un des bords du copdon, celui qui est le plus rapproché des parois de la loge, est toujour's plus granuleux que l'autre; et si l'on en examine un tronçon traité convenablement et sous un grossissement suffisant, on observe toutes les transitions entre les cellules fusiformes normales qui occupent le bord interme et celles qui, se trouvant au bord opposé, renferment dans leur longueur tonte une série de granules très-l'éfringents. Ces dernières appartiennent bien cependant an cordun eentral et primitivement elles ne différaient pas des autres.

N'aurait-on pas à invoifuer la forme des celhules qui composent le cordon central et qui n'est celle d'aueun élément nerveux conmu, ces variations dans leur aspect et leur contenu non-senlement dans des régions différentes, mais sur le même point à différentes époques ne sont assurément pas de nature à éveiller l'idée d'un système nerveux.

Mais, je l'ai dit, ee n'est pas la structure intime qui a servi d'argument à Fritz Miiller pour motiver sa manière de roir, c'est bien plutôt l'apparence extérieure et notanment celle du plexus.

Plexus. - Le plexus n'est, dans l'espèce qui nous occupe, rien de distinet au fond du cordon central; cest un lacis plus ou moins compliqué de brides et de tractus. qui, se détachant du cordon central ou des ganglions, s'en ront rejoindre soit d'autres parties du système, soit les parois de l'endocyste. La structure histologique en

I Pl. Vil, fig. 6; pl. Vi, fig. $3 \mathrm{cp}$. 
est tonte semblable à celle du cordon principal, samf pent-ôtre en ceci que los granulations y sonl somvent phus aboudantes.

(“est un plexus, cola est rait, el je ne samatis lui dommen mu autre nom, mais est-ee un plexus nervenx?

Salisisons tout d'alond $n 1$ aven que Glaparede laisse échapper en déerirant le systène nervenx colonial de la bugule : "On ne lrourelait peul-cere pas deux loges, dil-il, où le plexus soil semblable à luimome!n

Quel système nerreux est celui-ci qui affecte des lormes aussi rariables? Ce que l'auteur génerois dil de la Bugule s'applique encore plus exactement à notre Vésiculaice.

Lit, en effet, dans nombre de loges il n'y a pas trace de plexus aulour du cordon central, et là où il existe il nallecte aucune rógularité?

Ce n'est pas senlement en différents articles ou en dilfórentes branches que sa forme varie, éest dans le mìne article à dilférentes éporpues. Dans l'espace de peu de jouls une portion du plexus s'est modiliée sensiblement sous mes yeux, puisque j’ai vu un de ses rameanx s'atrophice et disparaitre totalement. Dans une zoécie arant la formation d’un nouvean P'olypide et surtout pendant sa résorption, rien n'est plus changeant que la forme qu'il alfecte.

'Terminons enfin cette eritirgue par une dernic̀e obscrvalion. Dans tons les animaux donés d’un systeme neverix, les nerfs nut des papmolsts tixes, soit entre enx quand ils s'anastomosent, suit aree les organes auxquels ils se rendent et auplès desquels ils sont charegés de certaines fonctions, soil molrices, soit sensitives.

Nons venons de voir que pour leurs anastonoses clles nout alssolument rien de constant. En est-il autrement de leurs lapponts arec les organes?

Nullement. Les branches du plexus ne se remblent pas à folle ou telle partie du Iolypide ou de la zoécie, aux muscles rétracteurs ou extenseurs par exemple, mais on les voit au contraire se dirger vers des points indélerminés des parois de la loge, où assurément il n’y a ni montement à déterminer ni sensalion à perceroir, et là, contractant arlhérence arec l'endocýste par une large surface elles s'y perdent, s'y soudent, s'y londent, mais n'y forment rien qui ressemble à une terminaison nerveuse ${ }^{3}$.

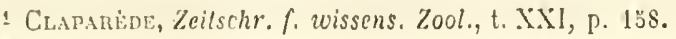

2 Pl, VII, fig. 1 et 2.

3 Pl. XXIV, fig. 2. 
D. Remarques générales. - Conclusion. - si l'examen altentif de la structure intime du cordon eentral el de ses dépendanres n’a pats été farorable à la théorie du systeme nerveux colonial, nous royons maintenant que la connaissance plus parfaite de ce que j’ai appelé les apparences extérieures l'óbranle encore davantage.

Deux faits anatomiques semblaient en effet surtout militer en finveur du système nerreux colonial, le plexus et les ganglions.

Sans parler de sa taille rraiment démesurée, le plexus, nous renons de le roir, présente dans sa forme el dans ses lapports ume rariabilité qui n'est véritablement pas compatible arec la notion d'un sỵstème nerreux et ses branches se rendent le plus sourent aux parties qui ont le moins besoin de nerfs.

Quant aux ganglions, nous sarons qu'on ne doit pas leur conserver mème ce nom, puisque, loin d'ètre des dilatations du cordon central, ils sont presque coupés en denx par un diaphragme qui ne laisse entre leurs deux moitiés qu'une étroite communication.

Enfin, un dernier argument tiré de l'anatomic me reste à opposer à la théorie du système nerreux colonial des Vésiculaires.

Le funicule est tellement semblable au reste de resystemo nerveux par sa forme, par sa taille, par ses rapports arec le ganglion basilaire, que Fritz Müller n’arait pas hésité à le considérer comme une dépendance de ce système.

Ce funicule a de plus tout à fail la meme structure histologique ${ }^{1}$ que le cordon central: il lui est done à tous égaris comparable.

Or', nous sarons qưil est éminemment contractile.

Est-ce là une propriété des tissus nerreux?

Plusieurs de ces faits et notamment la nature des ganglions et la structure histologique du cordon sont si faciles à obsepver et molirent si pen la théorie du système nerreux colonial, je dirai mêne qü̈ls ont tant de peine à y cadrer, quou est nalurellement lenté de chereher quet est l'argument puissant qui a lait passer Fritz Muiller par-dessus ces difficultés.

Cel argument, il me semble le trouver avee la plus grande évidence au début du mémoire du sarant allemand et dans la méthorle mème qu'il a suirie pour exposer' ses idées.

Frappé des mourements simultanés que les dirers membres d'une colonie de Bryozoaires exécutent sourent simultanément, et dont, 
pour ma part, jo veruis facilement l'explication dans une canse conmune agissant en môme temps sur tous, l'auteur se demande si cos mourentents d’ordre générial et supérieur ne procèdent pas de phus hat que des loges individuelles; il pernse qu'il toit exister un organe de transmission qui eommande tous ces mourenents, en un nut, un syclime nervenx colonial, et il le cherehe.

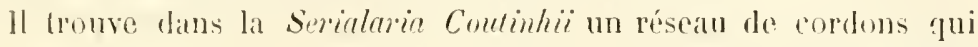
relient les individus, m plexus, quelque chose qui ressemble ì des ganglions; cela suffit, éest plus qu'il n'en faut; il s'en empare, il en fait un systeme nerveux, il prédit qu'on trouvera semblable chose dins les autres Bryozoaires; on tronve bientot en elfel quelque chose d'analogue dans les Cheilostomes, et sa théorie, confirmée d'une maniẻréclalante, est acceptée sans conteste jusqu à ce que des objections très-sérieuses s’élèvent contre clle.

C'est done, on le voit, un fait physiologique qui a fait concevoir à Fritz. Müller, je suis presque tenté de dire è priori, la nécessité d’un systeme nerveux colonial.

Examinons ce fait.

Je dois dire que je n’ai jamais été témoin d'ancum de ces mouvements d'ensemble exécutés par tous les individus d'une colonie, qu'il ne me parùt avoir pour cause quelinte choe on quelque aceident de niture à frapper simultanément tous les individus.

Mais, quelque opinion qu'on ait à cet égard, reste à déterminer si c'est bien par l'entremise du cordon central que se fait la transmission des sensations, et si cet organe est réellement capable de remplir me telle fonction.

C'est ce dont j’ai essayé de me rendre compte par l'expérience suivinte :

Je choisis dans une de mes curettes une branche bien vivante de Bowerbankie imbrirata récemment arrivéc de Roscoff.

Un Polypile était en plein épanouissement à son sommet.

J'approchai de la branche des ciseanx fins. Le seul mourement de l'eau fit reutrer l'animal dans sa loge.

Peu de temps après, il sortil ì nourean, je repris mes ciseaux et, cette fois je pinçai aree soin la branche.

Inçuićté par ces mourements, le zoüde se rétracte, mais bientùt il ressorl, puis rentre pour' s'épanouir encore.

Enfin, habitué sans doute anx petites seenusses que par l'intermédiare des ciseans lo tremblement de ma main déterminait 
dans le rameau, il resta épanoui pendant près d'une demi-minute. Je fermai alors doucement mes ciseaux bien aiguisés, la branche se détacha, et, en la suirant à la loupe, je pus m'assurer que, ni pendant que j'en opérais la section, ni pendant qu'elle tombail au fond du rase, le Polypide ne s'élait rétracté.

11 demeura au contraire épanoui au fond de la cuvette, témoignant bien par là qu’il était resté tout à fait étranger à l’opération qui le séparait de la plus grante partie de la colonie.

Je portai ensuite la branche coupée sous le mieroscope, et je eonstatai que la zoécie oceupée par le Polypide en question recerait bien un rameau du cordon central qui arait été sectionné.

Un organe qui ne transmet pas aux parties auxquelles il se rend le sentiment d'une lésion aussi considérable mérite-t-il le nom de sys. teme nerveux?

Il y aurail encore beaucoup d'arguments à faire raloir contre la théorie du systeme nervenx colonial, mais, comme ils seraient surtout tirés du rôle et de la nature de ce système, ils troureront micux leur place dans le prochim paragraphe.

Pour le moment, me londant sur les faits tant anatomiques que physiologiques que je viens d’exposer, je me borne à déclirer que je ne puis, en aucune manière, le considérer comme un système nerveux colonial.

\section{$\$$ 2. Importance, fonctions et attributions du système nerveux colonial des Bryozoaires.}

Nous venons de terminer le chippitre qui précède par cette conclusion que le prétendu système nepreux colonial de la Borerbankire imbricata n'avait rien de nerveux.

Cette conclusion estelle applicable aux autres Bryozoaires? Le système nerveux des Cheilostomes est-il l'homologue de celui de la Bowerbankia?

A cette demantle, tous les auteurs ont déjà répondu affirma tirement.

Sans doute le système en question ne se présente pas partout avec la même forme; dans la plupart des Cheilostomes, il est impossible de distinguer un cordon principal, touts'y réduit ì un plexus, mais celil tient miquement an mode de grvupement des loges et à l'absence de Irone common.

En effet, dans lés espèces où les loges se suecèdent sur une seule 
file formant quelque elose d'analogue ì un stolon, dans l'Anguinaria spatulata, par exemple, on roit ce stolon pareouru par un cordon principal duquel se détachent les funicules des polyjpides suceessifs, et aux points où deux articles se joignent, le cordon s’épate sur les deux fices de la cloison qui les sépare, formant, conme dans la Bowerbankit, une apparence de ganglion.

Lexamen histologique nous démontre eneore mieux l'homologie, ou plutòt lidentité des deux ohjets. Dans la Bicellaria, dans la Bugule ${ }^{1}$, dans foutes les espères que jai étudiées, j’ai rencontré les mèmes cellules fusiformes que dans la Bowerbankia; il n’y a de rariations que dans la taille des cellules et dans le nombre et la nettelé de lemrs noyaux. - J'aurai occasion de revenir plus tard sur les direrses formes que peut prendre le système nerveux colonial ehez les différents Bryozoaires; pour l'instint, je me borne à constater qu'au point de vne anatomique et histologique, il ny a lieu d'établir entre elles aueune différence fondamentale.

Aussi bien, la physiologie nous conduit aux mêmes eonclusions. Il m’est plusienr's fois arrivé, voyant bien épanouis an fond de mes euvettes des gronpes de Celleporat on d'Aleyonidium, de toncher sueeessirement avee une aignille la conronne tentaeulitre de chacun des lolypides. Ils rentraient tous isolément dans leur loge sans (qu'il y ait eu en rien communication d'impression; an contraire, ils se rétractaicnt simultanement au moindre ehoc.

Si ce quion a désignís sous le nom de système nervenx colonial ne mérite pas ce nom, qu'est-ce done que ce plexus, cet ensemble de cordons reconnus chez tous les liryozoaires, quel est le rôle de ce şrstìne, quelle est sa malure, quelle est sa signification morphologique?

Ponr répondre ì cette nourelle question, il fant reprendre de plus près l'étude de la structure intime dis tissu qui le compose.

A. Origine des cellules flottrntes (Fettiroppar). - Que l'on examine, soit a lećtat frais. on mieux après traitement par l'acide osmique faible le corton principal dans une branche de Bomerbankia imbricrea ${ }^{2}$ on de Vesicularin spinusa; on reconnaîtra avec heaucoup de facilité les rellules fusiformes qui le constituent, et, pour pen 
que l'examen se sontienne el que l'on observe le cordon sur une certaine Jongueur et dans une région où il soit bien transparent, on trouvera certainement en quelque point des cellules plus ventrues que les autres. 11 m'est arrivé plusieurs fois, comme mes plancles en font foi ${ }^{1}$, de tomber sur des endroits où l'on pourait observer sur le cordon toutes les gradations dans la forme des cellules, depuis la simple cellule normale fusiforme et étroite, par la cellule rentrue, jusqu’a celles (qui, boursoufflées el arrondies, prêtes à se détacher, ne tiennent plus au cordon que par une de leurs extrémités.

11 est impossible de constater ces transitions et de roir la manière dont ces cellules arrịent à se déformer et à se séparer du cordun sans les comparer aux celiules de même trille quion voil flotter dans le liquide qui remplit les zoécies ou les articles de tiges et que les auteurs anglais désignent sous le nom te floating cells ?

Ces cellules, qu'on les considère au moment où elles sont prêtes à se détacher du cordon, ou qu'on les premne libres dans le liquide ambiant, se composent d'une membrane mince el transparente affeetant une forme arrondie ou légèrement orale et contiennent le plus sourent un, deux on plusieurs granules de très-petite laille et très-réfringents.

Cette catégorie de cellules flottantes n’est pas particulière aux Vésiculaires, j'en ai trouré de tontes semblables dans la Bicellaria ciliata ${ }^{3}$ la Bugula avicularia et plusiemrs autres espèces.

Ce ne sont d'ailleurs pas là les seuls corpuscules flottant dans le liquide des loges qui tirent leur origine du prélendu système nerreux colonial. On rencontre encore dans l'intérieur des articles de tige de la Bowerbanlia imbricata des cellules à peu près comparables aux précédentes pour la taille et pour la forme, mais dont la carité, au lieu d'ètre occupée par un contenu clair ne renfermant que quelques. granules disséminés, en est au contraire eomplétement bourrée. Ces cellules se forment de la même manière que les précédentes, mais dans les régions où le cordon est lui-mème surehargé de gramules. II arrive souvent que la membrane de ces cellules se déchire el laisse échapper les noyaux qu'elle contenail, rui se trourent de la sorte isolés dans le liquide cavitaire et ordinairement collés aux parois de la loge.

\footnotetext{
1 Pl. XI, lige. !.

2 Pl. X, fig. 8.

3 Pl. X, flg. 10 ,
} 
Voici encore une espèce de coppusenles flottants qui est trìs-généralement répandue chez les Bryozoaires. La Lagenella mulans ${ }^{1}$ en a ses loges remplies, mais c'est surtout che\% les Cheilostomes ${ }^{2}$ qu'on la rencontre. Ce sont des granules très-réfingents, transparents, qui, an lien dètre arondis comme ceux que nous venons de volu et renfermés dans lintérienı de cellules, sont libres, plus on moins anguleux et ressemblent ì des noyanx de cellules.

On les voit tantôt flotiants, fantôt suspendus à des filaments qui dépendent du sỹstème nerveux colonial et serrent soit à rémir diffé. rents rameaux du plexus, soit à ratlacher ees mênes rameanx aux parois de la loge. Ces granules sont en quelque sorte un épaississement de ces filaments en certains points, une sorte de condensation ì ume place déterminée du protoplasme rui les constitue, et lorsque le filment vient à se rompre en dessus et au-dessous, ils deviennent libres el flotlent dans la cavité générale. D’autres fois ils résultent de la transformation en place des cellules du cordon ou de ses dépendinces. Toujours est-il que dans tous les cas, dans les zoćcies adultes, ils dérivent toujours du prétendu système nerveux colonial.

Cette espèce de corpuscule flottant est extrèmement réprandu chez les Bryozoaires; on en troure de semblables, et se formant de la mème façon, chez les Bugules, l'Eucratea, l'Anguinaria et bien d'autles.

Nous sommes done en droit de dire en terminant que plusieurs des corpuscules flottants que lon peneontre dans le liquide cavilaire des loges adultes dérivent du système nerveux colonial dont ils ne sont une tles éléments transformés.

Smilt ${ }^{3}$ a fait une étude spóciale de ees eorpuseules et de lemr ròle, mais il n'avail pas mis en relief leurs rapports avec le prétendu systeme nerveux tont une des fonctions, nous le royons par là, est te produire des corps (qu'on peut assimiler aux globules sanguins des animanx infériens.

B. Déceloppenent des éléments reprortucteurs. - Le même auteur sućdois fait entre les granules de Fettliroppar ou corpuscules sanguins el les élóments reprodnctents màles un rapprochenent qui a étri discuté, mais gui se tromve, comme nous allons le voli, parfaitement fondé.

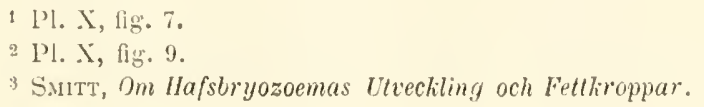


Je prendrai pour premier sujet d'étude la Ialkeria cuscuta, qui, à cause de sa transparence, se prête à merreille à ce genre de recherches.

Le bourgeon est à peine ébanché, on commence seulement à reeunnaître les saillies 'gui deviendront les tentacules, et déjà dans le sein du funieule nettement accusé à cette éporque, ljien ru’il soit sonvent encore accolé sur une eertaine longueur aux parois tle la loge. il se fait une prolifération active des cellules qui, ì mesure qu’elles naissent, sarroudissent eomme lescellules flottintes ordinaires et, se repoussant les unes les autres, finissent par s'acenunuler et constituer autour du funienle une masse framboisée de formo irrécrulière ${ }^{1}$. On distingue dans chacune d'elles, lorsqu'elles commencent à mùrir, un, denx ou trois petits noyaux clairs, et e'est seulement lorsque le Polypide touche à son développement parfait qu'elles se détachent pour tomber dans la carité de la loge et finissent par laisser à décourert lo tissu central et normalement constitué du funienle. Elles se séparent une à une ou plus somvent par paquets et le ou les noyans quion apercerait dans ehacme d'elles, devents des Spermatozoüdes parfaits, eommencent à sortir de la cellule mère en passant d'abord leur' quene à travers la paroi perforée de leur prison ${ }^{2}$. Lacenmulation de cellules mères se fait à peu près également sur toute la longneur du funicule; cependant reps le hant elles sont plus clair semées, de sorte quion remarque généralement assez bien tout au semmet et au conlact du ereum stomacal ${ }^{3}$ denx cellules qui, comprises dans une enveloppe commme, prennent de bonne hemre une taille un peu supérieure à celle des antrés, dont elles n’élaient pas distinetes primitirement. Elles sont empatées, ponr ainsi dire. dans le tiss: du funicule; re sont les rudiments des reufs.

plus d'un ohservateur a ru arant moi les cellules mères des Zoospermes se développer sur le funicule; Van Beneden les a figurées en Isit dans sa belle monographie de la Luguncula repens et lont le monde sait que éest sul le mìme appendice que se déreloppe le testicule des Bryozoaires d'ean donce. Il n'est done pas étonnant que j'ate pu roir'se former absolument de ha mème manière les cellules mères dans le sein du funicule thez un certain nombre d'espèces

\footnotetext{
1 P. X, firs. A.

2 P]. XI, fig. 10.

$3 \mathrm{Pl} . \mathrm{X}$, fig. ¿ ov.
} 
variées, la Bicellaria ciliata, la Bourerbantia imbricata, la Lepratia Martyi.

Il est an contraire admis généralement que les onufs se déreloppent vers le sommet de la loge sur les parois de l'nulocyste, comme cela a lieu chez les bryozoaires d'ean donce. Ils ont été ligurés dans rette

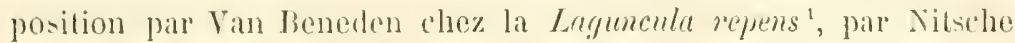
chez la Bicellaria ciliata ${ }^{2}$, par Snitt chez la Scrupocellaria sanpese ${ }^{3}$. II peul done paraître surprenant que je présente iej les wufs d’une Vésienlative comme se développant dans le sein même du lunicule. Rien cependant n'est plus exact et je les ai observés maintes fois ì cette platee. J'ajoute que le développement de l'auf dans te funicule n'est pas un fait isolé ni partienlier à la V́allieria cuscuta, comme on rit le roir.

J'ai olsservé les aufs dès l'époque où ils ne sont eneore qüindiqués, el suivi leur développenent dans les sept espèces suivantes: Bowerbmtia imbricata, Lagenella mutans, Vallieria cuscuta, Bicellarit ciliata, Bugula flabellata, Bngula avicularia, Lepralia Martyi, ot par. tout jai pu constater avee la dernière éridence que des aufs naissaient dims le sein mème du funieule, ì còté dés Zoospermes, et dans la dépentance du Polypide.

J'ai déjà indịué comment les choses se passent dans la Vallievir cuscuta, où les faits ne permettent aueun donte. Its sont tout semblables dans la Bonerbankin imbricata et la Layenella mutans, et le mode d'origine, aussi bien rue le mode d'éracuation de la larre, sont tellement caractéristiques, comme on le verra plus loin, qu’il est impossible de s'y méprendre.

Dans la Bicelluria ciliatn on remarque presque en tout temps andessons dn cireum stomacal un corps oroüle transparent ${ }^{5}$ que Ilinclis a mème indiqué sans en connaìtre la signification dans la figure que j’ai reproduite (pl. V'lII, tig. 10). Ce eorps est mue dépen. danee du funicule aux dépens de la substance dhquel il est constitué.

Ur, ce n'est autre chose que l'oraire et e'est dans son sein que se forment les reufs. Ia matse se divise en deux tout d'abord, les deux moitiés restent longtemps accolées, mais l'une d’elles prend rapide-

1 VAx Bexedex, Recherches sur les Laguncules, pl. I.

2 Nirscul, Zeitschrift, liand XX, pl. 1, fig. Lí ve.

3 sint, Om llafstryozvernas l'teeckl., p!. VII, fig. 1 u.

: PI. X, lig. 3, ov.

I'I. X, fig. 1, ov. 
ment un accroissement plus grant et une teinte grise, elle derient finement granuleuse et de plus en plus opaque; enfin elle se détache du funicule et on la voit cheminer rer's le haut de la loge, se rendant sans, doute dans l'ovicelle. La masse qui était restée en retard se développe à son tour, à moins qu'elle ne subisse une nouvelle division, car jai sourent ru dans une même loge deux aufs en roie de maturation intépendammeut de l'oroüde hyalin qui persistait encore. Ces wufs ont un vitellus assez fortement granuleux et opaque pour masquer la résicule germinative; il est cependant impossible de douter de leur nature orulaire guand on en voit, ce qui un'est arricé souvent, dans l'ovicelle à tous les états de développement, depuis le moment où ils ne sont eneore segmentés qu'en deux sphères jusqu'à celui où, passés à l’état de larre, ils sont prìts à s'échapper.

Dans la Bugula avicularia, Claparède avait déjà signalé les rapports des oufs arec le funicule, la masse orarienne se téveloppe comme cet auteur l'a reconnu de très-bonne heure; mais, dès le lébut, c’est dans le sein du funicule qu'elle se eonstitue el tout au sommet de cet organe '; c'est à la mème place que les coufs se produisent suceessivement au nombre de deux ou quatre par des divisions successives ${ }^{2}$. Dans cette espèce les aufs ont de bonne heure une teinte rosée, leur contenu est phus char que dans l'espèce précédente et on distingue nettement la vésicule et la tache germinative.

Dans les deux espèces, le même funieule qui produit à son sommet des rufs, développe dans la partie inférieure des Spermatozoüles.

Dans la Bicellaria ciliatu particulièrement les faits sont faciles à observer et, tandis que l'reul' se développe vers la partie renflée de la loge, e’est dans la région infériuture et étroite que les cellules du funicule subissent cette transformation particuliere qui en fait des cellules mères de Zoospermes ${ }^{3}$.

Dans la Lepralia Hartyi l'ovaire, qui est volumineux, se trouve précisément à la place où le funicule communique avce le système nerreux colonial des zoécies voisines et, ayant observé un très-jeune

1 Dès 183̈6, IIuxley (Note on the Reproduct. org. of Cheilost Polyz., dans Q. J. micr., sc.,t. IV, 1). 191) a décrit la formation de l'ouf et des Zoospermes dans le sein du lunicule de la Bugula avicularia. L'ouf, d'après lui, est attaché immédiatement audessous de l'estontie du Polypide ot presente deux taches germinatives. On voit par lik que cest l'ovaire entier, aree les deux onfs qu'il contient, que l'auteur anglais a pris pour un ceuf; mais, ì part ecla, sa description est très-exacte.

2 Pi. X, fig. :s et 6 .

${ }_{3}$ Pl. X, tig. I, $\mathrm{cm}$; pl. Xl, fig. ๖., $\mathrm{cm}$. 
nvaire, j’ai puroir tistinctement le funicule se détachor de sa surface pour gagner le corps brun ì cóté duquel se dévaloppat un jeme bolligeon ${ }^{2}$.

C'est done entore dans la substance du prétendu système nerreux colonial que lovaire se déreloppe chez la lepratiu Hartyi. Comme dans les espèces précédentos, les oul's y naissent par paires, mais de la manière suivante:

La masse ovarienne primilive se divise en demx porlions, l"me reste tout d'abord rudinentaire, l'antre an contraire se déreloppe et se ereuse d’une cavilé transparente dans lafuelle apparaissent teux œufs dont l'un prend un développement très-rapide, tandis que l'autre attend pour se parfaire l'entière maturation du premier ².

Le testicule, dans la mène espèce, Jont les zoécies sont d'ailleurs, jai de fortes raisons de le croire, unisexućes, se déreloppe à la même place que l'oraire et aux dépens du même tissu funiculaire; on roit nettement à travers les parois transparentes de la loge les Zoospermes passer leur queue à travers des cellules rondes qui ressemblent absolument aux cellules flottantes du liquide cavitaire ${ }^{3}$.

En présence de ees faits, que dirai-je des observations de Van Beneden, de Nitsche et de Smitl, qui figurent les oufs au sommet de la loge dans trois espèees dilférentes?

Je suis resté lougtemps dans le doute au sujet des observations de Van Beneden, car une Tésiculaire fréquente à Roseoff, la Lagenella mutans, me paraissait se rapporter aux descriptions du sarant prolesseml de Lourain et jy arais vu le déreloppement de l'ouf' se faire d'une manière bien différente.

Je dois à l'extrême obligeance de M. Ed. Van Beneden, qui a lyien roulu recueillir et m’envoyer d'ostende des échantillons de Lağuncules, dètre sorti de ce doute.

Les Lagruncules que j'ai reçues de Belgique conservées dans l’alcool étaient en pleine reproduction et j'ai pu coustater la parfaite exactiturle des dessins et descriptions de l'auteur. Les oufs sont dans l'adulte sur les parois de la loge, ils sont de fort grande taille et assez nombreux, et il semble, lorsqu'on examine l'oraire dans cet étal, qu'il ne puisse y avoir le moindre doule, et que réellement les reufs se développent sur lesparois de la loge et aux dépens de l'endocyste.

\footnotetext{
1 Pl. XI, fig. $3, f n$, ov.

2 Pl. XI, fig. (i.

9 Pl. XI, fig. 10.
} 
Les idées changent au contraire si l'on examine le bon'geon. Vau Beneden eroit que les Zoospermes ne se déreloppent que sur le funienle du Polypide adulte. Cependant j’ai vu avee la plus grande netteté la matsse des cellules mères au-dessus du bourgeon à peine ébauché, elle est toute semblable à celle qu’on olsserve dans les autres Vésiculaires (fig. 1).

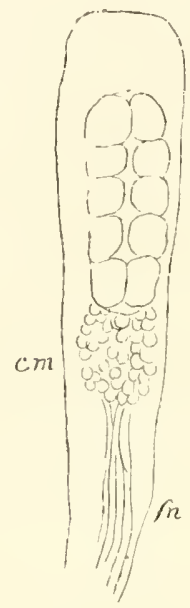

Fu. 1. - Jeune bougenn de Lagmevla repens. V. B. pre funieule; cm, cellules untes des zonsprermes et des urufs non encore diftireneies at se développant dans le sein de ce cordon.

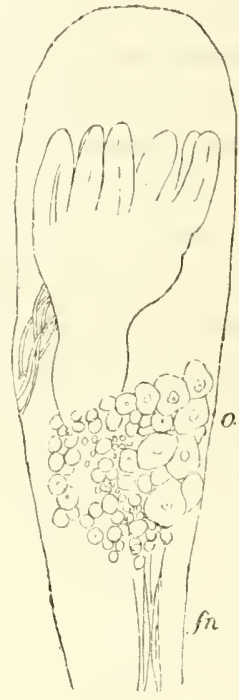

Fig. 2. - Bomroenn plus araneé ; les unfo qui weviennent prugressivement distincts des cellules meres de zorosiermes, s'itulent sur la paroi de la loge.

A cette éporque il n'y a pas trace d'ovaire, mais la masse des ecllules mères embrasse presque toute la largeur de la loge et tonche les parois.

C'est alors, comme le montre la figure 2, représentant un bourgeon plus avancé, c’est alor's qúe les cellules mères les plus rapprochées du Polypide prennent un accroissement spécial et une forme particulière; il se fait en cette régrion une prolifération actire de cellules, et le tissu constitué par leur ensemble s'étale sur les parois de la loge, $\mathrm{J}$ contricte adhérenee, et lorsque le tissn du testicule commenec à s'atrophier par suite de la fonte de ses cellules et de la mise en liberté des zoospermes, alors seulement il y a discontinuité entre le funicule et l'ovaire, qui, comme on le voit, en provient primitivement et n'a pas une autre origine que le testicule. 
Ce cas rentre done encore dans la loi générale; mais, par le nombre de leurs ceufs et par le mode d'évacuation que vau Beneden a décril, aussi bien que par la constitution de lapprareil operenlare, les laguncules n’en constituent pas moins, dans la fimmille des Vésiculaires, un lype tres-pemarpuable et tout à fait a part.

les olserritions de Nibshe et de smitt ont un tel caractire de précision, qu’il est impossible, quand on a lu les mémoires de ces itu. teurs, de douter le l'existence d'une paire d'œul's vers le sommet de la loge dans la Bicellaria ciliata et la sempocellarin serupnsa.

J'ai, d’ailleurs, observé le mêne fait dans la Membraniprna membranacert. An mois de mai, les loges étaient bourrées de spermatozoïdes. Sur l"une des parois latérales de chaque loge et rers le bas, on observait un amas de cellules à noyaux clairs : e’était le testícule, fort semblable d'ailleurs à celui de la Lepralia Martyi.

Sur l'autre paroi el rer's le haut, on distinguait un ouf granuleux assez opaque.

L'ovaire, comme le testicule, élait an niveau de ees plaques criblées, à travers lesquelles passent d'une loge à l'autre les fibrilles du système nerveux colonial, et qui sont à peu près semblables dans cette espèce à celles que Nitsche a décrites sous le nom de Rosettenplatte dans la Flustra membranacea.

Gue dirai-je maintenant des cufs parićtaux de la Bicellaria ciliata, après avoir décrit comme je l'ai fait les auf́s funiculaires? Pendant tout l'été, depuis mai jusqu'en aoùt, je n’ai jamais vu que les nufs funiculaires. Je les ai vus à tous les états de déreloppement, depuis le moment où ils diffèrent à peine d'une des cellules du funicule d'un jeune bourgeon jusqu'à celui où, devenus voluminenx el granuleux, ils cheminent ver's le haut de la loge, depuis le moment où ils sont encore reconnaissables dans l'ovicelle, dès le début de la segnuentation, juscu’à celui oì ils sont transformés en larves ciliées. Je n arais pu trouver aucun ouf pariétal, lorsque en septembre j'eus la bonne fortune de reiseontrer sur une mème branche les denx espèces d'œufs'. J'en conserre la préparation. Les œufs pariétaux sont trèsnéts et ressemblent parfaitement à ceux (que Nitsche a figurés ${ }^{2}$. Par malheur, l'époque arancée de la saison ne m'a pas permis de poursuivre plus arant l'étude de ces oufs et de leurs transformations.

2 Pl. XI, fig. $i$. 
Je ne puis que domner pour ee qüil est ce fait qui me paraît inconlestable; li Bicellaria ciliata produit deux espèces d'wufs; les uns dans le sein du funicule, les autres sur les parois de la loge.

Sous toutes réserves, je proposerai cependant les considérations suivantes:

Jo n'ai jamais vu d'eufs pariétaux au commencement de l'été; j’en ai, au contraire, olsservé un certain nombre en septembre.

Xitsche les donne, de son côté, comme se produisant dans l'arrière saison.

Les Bicellaria, d'autre part, sont déjà en pleine reproduction au commencement de mai et les oricelles bourrés de larve.

Les deux modes de reproduction ne se succèdent-ils pas?

En'second lieu, j'ai constamment vu que les zoécies contenant des œufs pariélaux étaient déponrues d'ovicelles, bien que plusieurs loges fussent adultes et les æufs bien développés.

Les zoécies pourvues d'un ouf funiculaire développé étaient, au contraire, "constamment accompagnées d'un ovicelle. Il est curieus que, sur li figure de Nitsche, la zoécie où il représente des œufs parićtaux manque justement seule d'ovicelle ${ }^{1}$. Est-ce un hasard?

Dans la Scrupocellaria scruposa, j’incline beaucoup à croire qu'il existe aussi deux sortes d'œufs, car je n'ai aucune raison de révoquer en doute les observations de Smitt, et cependant j'ai bien certainement ru des coufs se dérelopper au lond de la loge et au-dessous du Polypide au mois de juillet.

Tout en regrettant de ne pouroir cette année étudier plus à fond cette question, j’insisterai sur les remarques suivantes:

Ce n'est jamais sul la paroi externe de la zoécie, mais sur celle qui est en contact avec la seconde rangée de loges, que se développent les œufs, là où, dans la Scrupocellaria, Glaparède a démontré l'existence de communications nerveuses entre les deux loges adjacentes ${ }^{2}$.

Il en est de même dans la Bicellaria, aussi bien que dans la Membranipora pilosa.

Les ceufs pariétaux paraissent done avoir quelques rapports aree le système nerveux colonial. En l'absence de documents précis, je ne puis mavaneer davantage.

1 Nitsche, Zeilschrift, Band Ẍ. pl. I, fig.

a Chaparìne, Zeistschrift, Band XXI, pl. IX, fig. 1, c. 
Qnoi qüil eusoit, de lous les fails qui riemnent d’otre exposés, je suis en droit de lirer les conclusions suivantes :

Les éléments reproducteurs males se développent aux dépens du lissu du systeme nervenx colonial dans les sept espèces suivantes:

Bowerbanlia imbricata, Valkeriu cuscuta, Lagenella mutans, Laguncula repens, Bicellaria ciliata, Lepralia Martyi, Membranipora membianacea.

Les wufs sont produits dans le sein du même tissu chez les :

Bourerbantia imbricala, Valkeria cuscutu, Lagenella mulans, Laguncula repens, Bicellavia ciliata, Bugula avieularia, Buyula flabellata. Lepralia Martyi.

Des ceufs pariétaux existent chez la Bicellaria ciliata et la Membramipora membranacea, et sans doute chez un plus grand nombre d'espèces.

On peut déduire de là que les Zoospermes dans tous les cas, les ceufs très-sourent, peut-être généralement, se développent, comme les globules du liquide cavitaire, aux dépens du tissu du prétendu système nerveux colonial.

C'est un argument de plus contre la nature nerveuse de ce tissu.

G'est une mar'que de son importance dans l'économie du Bryozoaire.

C'est en dernier lieu une preuve que la théorie de Ed. van Beneden sur la distinction originaire du testicule et de l'ovaire ne saurait être appliquée aux Bryozoaires, puisque dans plusieurs espèces, et notamment daus quatre vésiculaires, nous royons que, loin d'être produits par deux feuillets différents, leséléments màle et femelle se développent côte à côte et clans le même cordon.

C. Développement du Polypide. - Nous avons vu dans la première partie de ce travail que Ilincks, résumant les opinions qu’il partage avec plusieur's observateurs, admethait que le renouvellement du l'olypide pourait se faire de deux manières dans l'intérieur de la loge :

$1^{\circ}$ Par bourgeonnement de l'endocyste;

2. Par déreloppement du corps brun ou germ-capsule.

Il peut paraître invraisemblable a priori que le Polypide, toujours semblable à lui-même, ait deux origines différentes. Nous savons, d'ailleurs, que le corps brun élant incapable par lui-même de bourgeomner, on doit admettre que le bourgeon se forme simplement à sá surface ${ }^{\text {. }}$. 
Les figures 1 el 2 de la planche XIII représentent, l'une, la première ébanche d'un Polypide se développant sur le corps brun dans lEucratea chelata; la seconde, un bourgeon déjà arancé se développaut sur le funicule dans une logge dépourvue de corps brun. Il suffit de les examiner pour se convaincre, en premier lien, que le prétendu bourgeonnement du corps brun dans cette espèce a pour siége véritable le tissu plus ou moins sarcodique qui l’enrironne et qui dépend du système nerrenx colonial; en second lien, que le bourgeon peut se développer directement sur le système nerveux colonial en l'absence de tout corps brun et indépendumment de l'endocyste, car le cordon principal dans l'Eucrutea chelata est suspeudu dans la loge et n'esi relié à ses parois que par des trabécules le même nature.

Examinons enfin les jeunes loges qui occupent l'extrémité des branches et dans lesquelles le Polypide n’est encore qu'ébauché; nous constaterons les faits suirants 1 :

La jeune loge a encore la forme d'une massue; son endocyste, riche en cellules, est encore en pleine activité végétative². A ce moment, le système nerreux colonial est déjà parfaitement reconnaissable: il a la forme d'un cordon de diamètre presque égal à celui de la loge à sa base. Il est encore formé de cellules fortement granuleuses; son extrémité supérieure, qui est arrivée à peu près à la moitié de la longneur de la loge, est arrondie.

Le sommet de la jeune loge s’élargit; en mème temps, un tractus de cellules claires surmonte comme un cône transparent l'extrémité du cordon ${ }^{3}$, c'est l'ébauche de la gaine tentaculaire; puis on roit l'extrémité du cordon s'élargir. Son contour, d'abord uniforme, se

1 Depuis l'époque à laquelle ce texte a été rédigé, a paru dans la Revue scientifique du 29 septembre une note do M. Barrois, dans laquelle cet observateur décrit avec exactitude ce cas particulicr du développement du Polypide dans l'Eucratea chelata. Je suis heureux qu'il soit arrivé aux mèmes conclusions que moi, mais je m’étonne qu'il prétende que mes observations confirment les siennes.

Comment aurais-je pu confirmer des résultats qui n’ont été énoncés que quinze jours ef publiés six semaines après que les miens eurent été communiqués à l'Académie et insérés dans les Comptes rendus?

A l'époque oì je fis paraître daus les mêmes Comples rendus (9 avril 1877) ma première note sur le système nervenx colonial, M. Barrois n'avait encore rien publié sur ee sujet; il fit, le 2i aoûl, au congrès du Harre, sa première lecture sur les rapports du bourgeon avee le lissu endodermique, et dès le 13 j’arais énoncé et défini complétement le rôle et les caractères de l'endosarque dans une note à l'Académie, qui n’étrit que le résumé des l'echerehes spéciales que je publie aujourd'hui.

2 Pl. Xl, fig. 4.

3 Pl. Xl, fig. 4, gl. 
creuse de plusieurs échanerures qui déterminent autant de lobes, lesquels sont les rudiments des bras. Le développenent embryomnaire n'a plus dès lors qu'ì s'acherer. Je n'en poursuis pas le détail.

Je nai jamais vu dans l'Eucratea chelata aucun bourgeon se former sur les parois de l'undoeyste, et, dams les trois cas que je viens d'exposer, il semble manifeste que le Polypide se forme uniquement et exchusirement aux dépens du système nerveux colonial.

En est-il autrement chez les autres Bryozoaires et devons-nous admettre que le bourgeon se forme tantôt aux dépens de l'endocyste, tantòt aux dépens du système nerreux colonial, ou bien aux dépens des deux conches? Je ne le erois pas, et j'espère montrer, par les exemples qui vont suivre, que le fait que nous venons de constater dans l'Eucratea chelata n'est point isolé.

Dans la Beania mirabilis, qui, à cause de l'isolement et de la transparence de ses loges, se prète à merveille à ce genre d'observations, j'ai pu constater, avee autant de netteté que dans l'Eucratea chelata, que le Polypide se dércloppait dans les jeunes loges aux dépens du cordon principal.

Dans les Membranimora memlranacea et pilusa, le bourgeon apparaît torjours à la base de la loge, au-dessus d'une de ces perforations qui mettent en communication les plexus pseudo-nerveux de deux zoécies successires; dès son plus jeune àge, on voit se détacher de sa partie supérieure des tractus de substance sareodique qui gagnent l'extrémité supérieure de la loge el constitueront plus tard la charpente de la gaine tentaculaire'. Cies trabécules sont diversement anastomosés entre enx et forment un lacis que Smilt ou Hincks baptiseraient éridemment du nom de plexus nerveux.

C'est donc dans la dépendance du plexus de la loge où il croît, et en communication arec echui de la loge qui précède que le bourgeon se constitue dans cette espece.

Dans les Lepralia Martyiet granifera, c'est encore sur le funicule que se déreloppe le jeune bourgeon, soit dans les jeunes loges, soit dans celles qui sont déjà pourrues d'un corps brun, et dans toutes les espèces de Cheilostomes que jai étudiées, j’ai toujours vu le boulgeon, qu’on prétend naitre de l'endocyste, se développer réellement

1 Voir les figures de Nitsche, dessinées d'après la Flustra memiranacea (Zeitschrifl, Band XXI, pl, XXXVl, fig, is et $6, t s c h)$. 
sur le système nerveux colonial, aussi bien que celui qui est censé provenirigles corjs bruns.

Le mode de développement de la gainne tentaculaire est particulièrement démonstratif; j'ai montré tout à l'heure comment elle apparaissait dans deux espèces de Hembranipores comme un ensemble de trabécules ressemblant absolument au plexus nerveux des auteurs; dans l'Eucratea chelata, nous avons vu qu'elle se présente comme un cône de cellules tramsparentes qui ont absolument l'aspect de celles qui composent les extrémités naissantes dn prétendu système nervoux; il en est de mème dans toutes les autres espèces de Cheilostomes et l'on peut poser en principe comme lémontré par l'étude du développement ce fait, qui résulte aussi bien, d'ailleurs, de l'examen de l'adulte, que la gaine tentaculaire est de même nature que le funicule, elle se développe de même, est constituée par des éléments fort semblables, et comme lui elle est éridemment contractile. Cetle propriélé contractile est facile à constater partout, mais particulièrement chez les espèces où le Polypide pent se retirer profondément dans sa loge. Dans l'Anguinaria spatulatr, par exemple, c'est grâce à la contractilité de la gaîne que, de la partie rampante de la loge où il se retire parfois, le Polypide peut remonter jusqu'à l'entrée de la zoécie.

La gaine n'est done pas, à mon avis, une inragination de l'endocyste; e'est, tant au point de vue de la structure et du déreloppement qu'à celui des propriétés physiologiques, une véritable dépendance du système nerveux colonial, dans le sein duquel, comme on le voit, le Polypide se trouve compris tout entier.

Je ferai remarquer, en terminant, que l'opinion que je soutiens sur l'origine du bourgeon n'est point en contradiction avec celle des auteurs, mais qu'elle donne l'explication des dissentiments qui se sont élevés entre eux.

Smitl et !Hincks, en effet, ont soutenu longtemps que, dans les Cheilostomes, le bourgeonnement s'effectuait unirnement sur la surface du corps brun et nullement aux dépens de l'endocyste; ils ont donné à l'appui de eelte opinion des figures assez démonstratives et n’out atmis, plus tard, le développemeni aux dépens de l'endocyste, que comme $u$ cas secondaire et beancoup plus rare que le premier.

Cette opinion troure son explication naturelle dans ce fail que che\% les Cheilostomes le cor'ps Irum élant toujours suspendu entre 
les mailles du système nerreux colonial, le Polypide qui se développe aux dépens de ee lissu parait bourgeonner sur le corps brun lui-mème.

Quant au bourgeonnement sur l'endocyste, tous les auteurs qui en ont parlé ont-ils bien fail, tant dans leurs observations que dans l'interprétation de ces observations, lit distinetion entre l'endocyste proprement dit et le plexus nerveux? je ne le crois pas, car ce plexus est difficile à distinguer dans les jeunes loges, et dans les anciennes, ses rapports aveee le bourgeon ne sont pas toujours faciles it saisir, et peurent échapper ì celui qui ne les observe pos spécialement.

Nitsche a toutefois parfaitement fait cette distinction ${ }^{1}$, et dans son étude du bourgeomnement des Flustres, il reeonnaît que le Polypide est suspendu au funicule et en relation arec le système nervenx colonial qu’il désigne sous les noms de Funicularplatte, funiculi laterales, couche fusiforme de l'endocyste, suivant l'endroit où il le considère. Seulement, il admet que le corps mème du Polypide se forme aux dépens de I'endoeyste proprement dit (Epithelialschicht) sans en donner d'ailleur's de preuve.

Salensky² a prétendu depuis que le jeune bourgeon se montrait composé de très-bonne heure de deux couches et que ces deux couches correspondaient l'une, la couche interne à l'épilhélium externe de la loge ou endocyste proprement dit, et l'autre à la couche interne de cette niême loge, couche qui n’est autre que le système nerveux colonial.

Je ne puis partager cette manière de voir; les observations que j’ai pu faire dans plusieurs espèces, spécialement dans l'Eucratea et notamment la préparation que représente la figure 1 de la planche XIII, m'ont toujours montré de la manière la plus nette que le bourgeon se formait exclusivement dans le sein du système nerveux colonial et n’était relié au début à l’endocy̧ste que par des trabécules appartenant eux-mèmes atl prétendu système nerveux.

Les deux couches se distinguent dès le début dans les bourgeons, mais elles proviennent toutes d'un seul et mème tissu qui se différencic par la suite el qui n’est autre que le tissu du système nerveux colonial.

Snivons d'ailleurs de près la formation du bourgeon d'une Vésiculaire, la Vesiculariat spinosa.

1 Nitscie, Zeitschrift, Band XXI, p. is6.

2 Salexskr, Zeitschrift, Band XXIV, p. 346. 
Les figures 11 et 12, pl. XII, montrent le même stade à deux grossissenents différents; c’est le premier indice de la zoécie.

Dans les deux figures, on roit que l'ampoule qui soulère l'ectocyste est doublée intéricurement d'une couche assez épaisse d'endocyste cellulenx. A l'intérieur sont'des granules qui se continuent sans interruption avee ceux du cordon principal de la tige auquel ils appartiennent.

Cependant, à mesure que l'ampoule saccentue, les granules s'accumulent à l'intérieur et deviennent de plus en plus nombreux (fig. 13). Lorsque la zoécie a pris la forme d'une petite poire, mais avant que le diaphragñe ne soit apparu, on roit déjà nettement (fig. 14) que ces granules se sont réunis en une masse arrondie qui n’est autre que l'ébauche du Polypide. Les cellules n'ont plus qu'à se multiplier et à se répartir en deux couches pour reproduire la ligure 13 ; un pen plus tard, apparait dans la masse la le premier indice de la formation d'une eavité digestive (fig. 17).

On voit par là clairement que la formation du Polypide dans une jeune loge de Vésiculaire ne peut point être regardée comme résultant du bourgeonnement de l'endocyste, qui ne participe en aucune manière directe à sa production. Lorsqu'on traite une jeune loge dans ces conditions par l'alcool, le bourgeon intérieur se sépare de l'endocyste de la manière la plus nette, pour rester uni au funicule (fig. 16).

Tel est le mode de formation du bourgeon dans une zoécie nouvelle; étudions maintenant la manière dont il apparait dans une loge ancienne lorsque le Polypide se renouvelle, et prenons pour exemple la Vallieria cuscuta.

On pourrait être complétement trompé, si l'on n'examinait qu’un bourgeon déjà avancé, tel que celui que représente la figure $\mathbf{3}$ de la planche Vi dans la Bowerbankia. A cette époque, en effet, il semble attaché à l'endocyste.

Pour se bien rendre compte des faits, il faut les examiner dès le début.

On constate alors :

En premier lieu, que jamais un bourgeon ne naît sur l'endocyste d'unc loge ancienne, si ce n'est en un point où s'attache quelque branche du funicule du Polypide précédent.

En second lieu, que la première ébauche du bourgeon est due à une prolifération des cellules de ce rameau funiculaire qui forment tout d'abord comme une conronne autour d'une cellule centrale ${ }^{1}$.

I Pl. XI, fig. 11, 12, 13. 
Cette ébauche est, sans aucun doute, appliquée sur l'endocyste, mais cette membrane est à cette époque et sur ce point compléte. ment dépourrue de structure; elle ne prut done pas vraisemblablement produire les éléments du Polypide, qui sont au contraire tout naturellement fournis par le tissu du funicule.

Si le bourgeon se développe sur la paroi de l'endocyste, si plus tard il contracte momentanénent arec cette membrane des adhérences intimes, cela ne doit pas nous surprendre.

Il est nécessaire, en effet, dans les Vésiculaires, que le Polypides’attache à la paroi et gagne de proche en proche le sommet de la loge, puisqu'il faut que ce sommet subisse ce travail particulier, qui a pour objet de repercer l'ouverture et de développer la nouvelle collerette. Si dans l'Eucratea rhelata et dans divers, Cheilostomes le bourgeon se développe au milieu même du cordon principal, rela se conçoit, car le eordon ne fait que traverser la loge, venant de celle qui la précède pour aller dans celle qui la suit, le bourgeon peut alors se développer à mi-chemin en face l'ouverture. Dans les Vésiculaires, il n’en peut être de même, le cordon funiculaire se termine dans chaque loge, son extrémité doit correspondre à l'onverture de la zoécie. Il faut donc que le Polypide se trouve au sommet en contact avec l'endocyste.

Je serais presque tenté de généraliser el de dire, pour terminer, que dans tous les Bryozoaires' le déreloppement du Polypide se fait aux dépens du prétendu système nerveux colonial, si les Pédicellines ne constituaient, au dire de Salensky, une très-sérieuse et très-frappante exception.

Cet auteur, dans un récent travail ${ }^{1}$, vient de chercher à démontrer que le bourgeonnement de l'appareil digestif, qu'il assimile au Polypide, se fait aux dépens de l'endoeyste. Je produis ici une figure assurément fort semblable aux siennes et dans laquelle le bourgyeon est encore réduit à cinq cellules ${ }^{2}$, mais ces cellules ne me paraissent pas être étroitement liées à celles de l'endocyste, et m'ont tonjours semblé aroir plus de rapports arec les cellules fusiformes du parenchyme.

Supposant même que l'opinion de Salensky soit fondée, comme nous allons voir tout à l'heure que le tissu dit neveux dérive directement de l'endocyste et que dans les jeunes bourgeons de Vesicularia spinosa les granules, aux dépens desquels se constitue le bourgeon, tout en ippartenant au systeme nervenx colonial, ne sont

1 SAlenskr, Entoproctes (.lnn. sc. nat., 6e série, 1. V).

2 Pl. XIII, fig. 9. 
que des cellules d'endocyste fraîchement détachées des parois, on peut dire que les deux cas sont fort roisins.

Quoi qu'il en soit, ștous ces faits, qui sont d'une constatation fort délicate, demandent de nourelles observations; je n'avancerai pas, pour le moment, de conclusions trop alssolues et dirai simplement:

Que dans l'Eucratea chelata les bourgeons sont toujours formés exclusivement aux dépens du cordon funiculaire.

Que dans les jeunes loges de Vesicularia spinosa j'attrilune au même tissu la formation du Polypide.

Que dans les loges anciennes de Vésiculaire le renourellement du Polypide me paraît se faire de même, sans qu'il me soit possible cependant d'affirmer que l'endocyste n'y prend aucunc part.

Enfin, que dans les Pédicellines, il reste à décider si le bourgeon est une production directe de l'endocyste ou si, comme ailleurs, il dérive du système nerveux colonial ${ }^{1}$.

\section{§ 3. Origine du système nerveux colonal. Ses rapports avec l'endocyste.}

Maintenant que nous possédons quelques notions sur le rôle et sur les fonctions du prétendu système nerveux colonial, nous derons chercher à connaître son origine et ses rapports avec l'endocyste.

Pour atteindre ce but, il faut tout d'abord étudier cette dernière membrane, qui jusqu'à présent était considérée comme le seul tissu fondamental du Bryozoaire, et dont la structure a cependant donné lieu à bien des discussions.

L'endocyste des Bryozoaires d'eau donce présente une structure assez complexe sur laquelle j’aurai à revenir un peu plus loin ; il ne peut pas être regardé dans son ensemble comme l'homologue de celui qui tapisse intérieurement les parois résistantes des loges des Bryozoaires marins, et qui se réduit à une seule couche. Nalgré cette simplicité, on a émis direrses opinions sur la structure de l'endocyste des gymnolicmes, les uns le regardant comme une membrane cellulaire, les autres comme une membrane anhiste.

Le plús généralement, on admel avee smitt que l'endocyste des Ectoproctes ne présente la structure cellulaire qu’aux extrémités en voic de développement.

- Au moment où nous meltons sous presse, nous recevons communication d'un trasail de llatscheck (Zeitschrifl dı 18 octobre) qui traite du bourgeonnement des Pédicellines; mais il ne détermine pas quelle est l'origine du tissu aux dépens duquel se forme le Polypide. 
Jepartage complétement celte manière de roir; dans tontes les espicees que j’ai examinées, en l'étudiant dans les zoécies adultes, et sur les points où il n'y a anem mélange aree les cellules du tissı dit nereur, je n'ai jamais pu trourer dans l'endocyste proprement dit aucune trace de structure cellulaire, quels que soient les réactifs employés, acides picrique, chromique, azotique, osmique. - La membrane, contractée par les acides, se détache de l'endocyste tout d'une pièce et se présente comme une couche de protoplasna honogène dans le sein duquel lacide osmique révèle parfois comme une sorte d'ornementation réticulée très-délicate qui peut indiquer une ecrtaine orientation dans le sarcode, mais quine pent pas être assimilé à une strueture cellulaire proprement dite. Aux extrémités végétatives, l'aspect est fort différent. Examinons par exemple le sommet d'une tige de résiculaire en roie d'aceroissement.

L'ectocyste y est très-mince et doublé intérieurement d'un endoerste fort épais formant une sorte de ealotte et présentant une strueture cellulaire très-nette ${ }^{1 .}$

Toute cette calotte sphérique est en effet constituée par des cellules juxtaposées qui ont grossièrement la forme de cônes dont les bases seraient tournées vers l'ectocyste, formant sous sa surface comme une espèce de mosaïque irrégulière, tandis que les sommets seraient dirigés vers le centre. Celles de ces cellules qui sont les plus rapprochées du pôle de la fealotte sont très-allongées, tandis que les antres sont de plus en plus courtes à mesure qu'elles s'en éloignent.

Toutes, les cellules centrales surtout, présentent un noyau net et sont très-réfringentes.

Comme les cellules centrales se multiplient sans eesse, celles des bords sont peu à peu repoussées, les unes se résolvent sur place en protoplasme pour former la couche homogène dont est constitué l'endoeyste des parties anciennes, les autres se détachent et se transforment en corpuseules flottants qui obseurcissent par leur nombre l'extrémité des tiges et y forment une sorte de parenchy̧me. Enfin il en est d'autres dont le contour s'atténue en arrière au lieu de s'arrềter brusquement, et ces cellules se terminent par des prolongements qui s'anastomosent entre cux ef forment la première ébauche du système nerreux colonial; on voit en effet ces cellules, qui sont d'abord 
disséminées, se condenser et se rapprocher ${ }^{1}$ peu à peu jusqu’à constituer un cordon dont la forme s'accuse de plus en plus nettement à mesure qu'on s'éloigne de l'extrémité.

Ces faits sont très-faciles à constater dans les Vésiculaires, parce que, dans l'article terminal de la tige, aucun Polypide ne vient masquer la structure; aussi ai-je choisi cet exemple comme type, mais j’ai pu constater que les choses se passaient de même dans beaucoup d'espèces.

Les prolongements postérieurs des cellules de l'endocyste primitif sont par exemple très-remarquables dans la Bicellaria, la Bugula, l'Encratea ${ }^{2}$; dans les Membranipores, on ne voit qu'assez tardivement les premiers vestiges du plexus.

Nous devons donc admettre que c'est aux dépens des cellules des extrémités régétatives que se forme le système nerveux colonial, qui est une dérivation de l'endocyste primitif.

Après avoir montré que le tissu qu’on désigne sous le nom de système nerveux colonial n'arait rien de nerveux; après avoir constaté quil jouait le principal rôle dans le renouvellement du Polypide, dans le déreloppement des éléments générateurs et dans la production des corpuscules sanguins, il devient véritablement impossible de continuer à le désigner par son ancien nom, qui n’a plus aucune raison d'être. Il faut lui en trouver un autre. Dès à présent, et dans l'étude qui va suirre, jemploierai le terme endosarque comme synonyme de système nerveux colonial, me réservant de justifier cette appellation comme conclusion de ce chapitre.

\section{\$. Morphologie de l'endosarque.}

On n’a jamais signalé le système nerveux colonial que dans les Ectoproctes, et, parmi cux, chez les Bryozoaires marins, et nous ne l'arons encore étudié que chez ces derniers.

Mais puisque l'endosarque est un tissu si constant dans ce groupe, si important à tous égards, n'y a-t-il pas lieu de se demander si dans les autres Bryozoaires, dans les Entoproctes, ou dans les Bryozoaires d'eau douce, il n'y a pas quelque chose d'analogue? 
Sur ces derniers, malheureusement, je n'ai jas de recherches personnelles à apporter, mais les excellents travaux de Nitsehe ${ }^{1}$ ont jeté tant de lumière sur leur strueture intime el l'ont décrite avec tant de détail el de précision, qu’appuyé par les faits reconnus de beaucoup d'observateurs, el guidé par ce que je connais des autres groupes, je puis peut-ôtre arancer quelques propositions que je ne dome d'aillem's que sous réserve de reeherehes ultéricures.

Tout le monde sait que les Bryozoaires d'eau douce ont un funicule, et c'est mème che\% eux que cet appendice a été tout d'abord décrit. Ce funicule est contractile, comme celui des Bryozoaires marins, et, comme lui, s’attache à l’extrémilé du exeun du Polypide. C’est dans son sein que se déreloppent les statoblastes et aussi les spermatozoïdes.

Est-il possible, en face de tous ces traits de ressemblance, de méconnaître, dans le funicule des Phylactolæemes, l'homologue de celui des gymnolames, et par conséquent l'une des parties constitutives de l'endosarque?

Mais, dira-t-on, le funicule chez les Phylactolæmes n'est pas en rapport arec celui des Polypides roisins, il n'y a rien de comparable à cet ensemble de cordons et de plexus qu’on avait appelé système nerveux colonial; ici, le funicule est un simple prolongement de l'endocyste.

Mais l'endocyste, répondrai-je, l'endocyste des Phylactolæmes estil comparable à celui des Bryozoaires marins? Tous les auteurs s'accordent à lui reconnaître une structure beaucoup plus complexe.

Voici, d'après Nitsche, de quoi il est constitué.

Il se compose de trois couches:

Une couche interne composée d'un épithélium ciliaire très-délieat;

Une couche externe appliquée contre l'ectocyste et formée de cellules polygonales ou cylindriques.

Entre les deux, une couche musculaire formée d'une membrane fondamentale homogène, servant de support à des fibres musculaires longitudinales et transversales.

Il me semble quion peut reconnaître dans la conche externe formée de cellules cylindriques ou polygonales l'homologue de l'endoeyste proprement dit des autres Bryozoaires.

1 Nitsche, Beitragezur Anat. und Enfwich di's Phylact. Reicnert, ot ne Bors.Rer. noxd, Archiv fur Anat., Mhysiut. und wissen. Hedicin, t. X, 186s, p. stiö. 
Aux extrémités régétatives, nous arons ru, en effet, que l'endoeyste des Bryozoaires marins est formé de cellules cylindriques, et nous verrons bientôt que l'endocyste des Pédicellines est une mosaïque de cellules polygonales.

La couche interne ou épithélium ciliaire ne paraît pas avoir d'analogue chez les Bryozoaires marins, dont l'organisation est plus simple.

Quant ì la couche moyenne, il me semble y reconnaitre des caracteres communs suffisants pour l'assimiler à l'endosarque des autres Bryozoaires.

C'est en effet, d'après Nitsche, de cette couche moyenne que nait le funicule, qui n’est formé que par un prolongement de la membrane fondamentale, contenant quelques fibres musculaires. Les connexions sont done tout en faveur du rapprochement que je propose, mais la structure ne l'est pas moins. N'avons-nous pas reconnu à l'endosarque, au funicule de tous les Bryozoaires marins, au cordon principal de l'Anguinaria des propriélés contractiles? La gaine tentaculaire, que nous regardons comme une dépendance de l'endosarque, n’est-elle pas elle-même une membrane homogène parcourue par des fibres musculaires plus ou moins imparfaites, mais toujours contractiles?

Enfin, n'est-ce pas aux dépens de l'endosarque que se constituent tous les muscles, et les fibres dont ceux-ci sont formés sont-elles autre chose primitivement qu'une des cellules fusiformes de l'endosarque?

La couche moyenne des Bryozoaires d'eau douce mérite donc d'être comparée à l'endosarque, tant pour sa nature que pour ses rapports aree le funicule.

Bien que je n’aie pas ru ces faits par moi-même, ils ont été si minulieusement fléerits, qu'il mia semblé possible de les interpréter, tant les conelusions qu'on en peut tirer me paraissent frappantes.

On serát peul-ètre surpris de voir l'endosarque qu'on connaît habituellement sous la forme de cordon au centre de la loge ainsi appliqué sous forme de membrane sur les parois. Ce n'est, cependant, pas lì un fait isolé, et on rencontre l’analogue chez quelques Bryozoaires marins.

Nitsche lui-même décrit dans la Flustra membranarea, sous l'endocyste proprement dit, une couche à cellules fusiformes, qui n'est autre que ce que tous les auteurs ont déerit sous le nom de plexus. Dans le genre Valleria, le lunicule seul se présente sous forme de 
cordon, comme d'habitude ; mais il n'y a pas dans la lige de cordon prineipal, et l'on distingue nettement les cellules fusiformes de l'endosarque, qui sont disséminées en phus on moins grand nombre sur les parois internes de l'endocyste el reliées entre elles par des prolongements; il ne manque en vérité qu’un épilhélium ciliaire pardessus pour constituer un endocyste à trois conches, comme celui de l'Aleyonelle.

Venons-en maintenant à l'étude des Entoproctes et commençons par les Pédicellines.

De quoi se compose une tige tle Pedicellina echinata?

D'un ectocyste ou cuticule sans structure;

D’un endocyste formé de cellules polygonales, disposées en mosaïque;

D'une couche de fibres musculaires longitudinales;

D’un parenchyme central.

Quant au stolon, il est conslitué de mème, avec cette seule différence ([ue les fibres musculaires y sont rares ou mème absentes.

L'ectocyste est une membrane assez mince, chitineuse, hérissée de piquants dans la Pedicellina echinata.

L'endocyste diffère par' son aspect, suivant le lieu où on l'examine. A l'extrémité des tiges, au-dessous du diaphragme qui en sépare les têtes, l'endocyste est épais, réfringent et composé de cellules trèsmanifestes et serrées l'une contre l'autre. Sur le reste de la tige, on ne distingue pas facilement sur l'animal frais la structure cellulaire, mais l'acide picrique rérèle nettement les contours anguleux de cellules qui dessinent sous l'ectocyste une véritable mosaïque '. Ces cellules paraissent, du reste, presque dépourvues de rie; elles sont léprimées et paurres en protoplasma.

Elles sont également risibles dans les régions anciennes du stolon, tandis ru'à l’extrémité les cellules de l’endocyste affectent absolu. ment les mèmes caractères et la mème disposition qu'à l'extrénité des tiges de Vésieulaires. Les cellules qui avoisinent le pôle de la calotte terminale sont très-allongées, el celles qui les suivent sont de plus en plus conrtes ${ }^{2}$.

Passons maintenant à l'étude du parenchyme. C'est dans le stolon qu'on peut l'examiner le plus facilement. Là, en effet, il n'est pas

2 Pl. XII, fig. 1. 
masqué par la conche de fibres musculaires serrées qui, dans la tige, forme un revêtement à l'intérieur de l'endocyste.

La figure 6 de la planche XII reproduit une portion de stolon, et on voit que le parenchyme se compose en majeure partie de cellules fusiformes qu'il est impossible de ne pas comparer à celles de l'endusarque des autres Bryozoaires. Ces cellules sont reliées entre elles par des prolongements plus ou moins rameux, qui s'entrelacent de diverses manières.

La manière dont se forme l'endosarque est tout à fait semblable à ce que nous arons va dans la Baterbantia.

Celles des cellules de l'endocyste terminal qui ne restent pas sur les parois tombent dans la carité du stolon non sans émettre des prolongements sarcodiques qui les réunissent entre elles; on les voit se déformer et s'allonger peu à peu jusqu'ì prendre la forme que nous leur arons rue dans les parties déjà anciennes du stolon.

Fin somme un stolon de Pédicelline est tout à fait comparable à une tige de Bou'erbankia dans laquelle les cellules de l'endosarque, au lieu de rester accolées les unes aux autres et réunies en un cordon séparé des parois par un grand vide, seraient disséminées dans toute la carité de la tige, làchement unies entre elles et fornant une sorte de parenchyme au sein duquel le liquide caritaire se troure réparti.

Quelle signification derrons-nous maintenant donner aux fibres musculaires de la tige?

Nous arons toujours vu jusqu'ici les fibres musculaires se constituer aux dépens de cellules fusiformes appartenant primitivement à l'endosarque; il en est de même iei, les fibres de la tige sont des cellules fusiformes transformées; dans les très-jeunes tiges on ne trouve eneore que du parenchyme au-dessous de l'endocyste, les fibres musculaires n'apparaissent que tardirement et on roit tous les passages entre elles et les cellules fusiformes.

Le parenchyme des tiges et des stolons des Pédicellines est done à beaucoup d'égards tout à fait comparable à l'endosarque des autres Bryozoaires. Quant à la manière dont ce tissu se comporte dans la tête de la Pédicelline qui est pour nous l’homologue de la zoécie, quant à ses rapports avec le Polypide qui pour nous est uniquement représenté par le canal digestif et ses amnexes, je crois devoir appeler l'attention des observateurs sur les faits suirants. 
Nitsche, dans sa belle monograplic de la Pedicellina echinata ${ }^{1}$, décrit et figure, partant de l'espèce de bouton qui surmonte le diaphragme, deux fibres dirergentes quil désigne sous le nom de P'arenshymstränge nach der Unterseite der Magens, et qu'il regatrde comme des parties renforcées du parenchyme du corps.

Ces deux fibres sont représentées sur une conpe qui sectionnerait transversalement le canal digestif. Mais si l'un examine par transpareuce l'une des faces latérales de l'animal, on roit que du bouton partent en dirergeant un cerlain nombre de fibres ou tractus qui passent sur la surface de l'estomac et ront toules se terminer rers le cul-de-sac qui termine en haut cet organe à côté de l'entrée de l'usophatge.

11 existe un faisceau de fibres semblables de chaque côté de l'estomae, qui se trouve ainsi enserré dans une sorte de collier. Parmi ces fibres un certain nombre semblent se terminer sur la surface de l'estomac, les autres aboutissent aux organes génitaux qui surmontent immédiatement le cul-de-sac.

Dans ces faisceaux de fibres qui ressemblent étroitement aux prolongements des cellules du parenchyme de la tige, ne doit-on pas voir l'analogue du funicule des autres Bryozoaires?

Ce serait, il est rati, un funicule double; mais il arrive chez plus d'une espece que le funicule principal est accompagné d’un ou deux funicules accessoires qui s'insèrent un peu plus haut ou sur un autre point de l'estomac; le fait de la duplicité du funicule n’anrait done rien en lui-même d'exceptionnel, et il est certain que ces faisceaux présentent dans leurs connexions aree les autres parties des rapports frappants avec le funicule.

Comme le funicule des Ectoproctes, le faisceau des Pédicellines part du diaphragme qui est l'analogue de la perforation cloisonnaire des Vésiculaires; comme lui il a des rapports avec les organes de la reproduction. Enfin nous allons roir que la partie de l'estomac sur laquelle s'insèrent une partic de ses fibres est.précisément l'analogue du cul-de-sac stomacal des autres Bryozoaires. Qu'est-ce qui caractétérise en effet le cul-de-sar stomacal des Ectoproctes? e'est le grand déreloppenent des cellules hépatiques; or', dans les l'édicellines e’est précisément dans cette région que les cellules hépatiques sont localisées. Cette région supéricure de l'estomac est d'ailleur's le seul

1 Nitsche, Zeilschrift, t. XX, pl, II fig. 4. 
cxeum de l'organe et l'on n'hésiterait pas à le comparer an crecum des Ectoproctes, si sa pointe, au lieu de regarder les tentacules, était dirigée vers le fond de la loge.

Il semble que ce cecum ait été mis dans cette posilion par une sorte de torsion de l'estomac et ait entrainé dans ce mourement le funicule et les organes génitanx. La position du testicule entre l'estomac et les tentacules est en effet un fait tout aussi inusité chez les Bryozoaires que la direction du cæcum dans le même sens, et c’est un fait remarquable que, malgré ce déplacement, ils conservent leurs connexions.

Dans le Loxosome des Phascolosomes, que M. Carl Vogt a étudié l'été dernier au laboratoire de Roscoff ${ }^{1}$, le savant professeur de Ge nève a décrit un endocyste sous une cuticule anhiste et au centre de la tige un parenchyme composé de cellules fusiformes et étoilées, assez semblable à celui des Pédicellines, quoique un peu plus làche, et auruel je ne puis m’empêcher de l'assimiler.

Pour toutes ces raisons, il me parait évident que le parencliyme des Entoproctes ne peut être comparé à autre chose qu'à l'endosarque des Ectoproctes.

coxclusios.

Par la première partie de ce chapitre, nous arons démontré que le prétendu système nerveux colonial n'a rien de nerreux.

Dans la seconde nous avons cherché ì comnaître quels sont le rôle et la nature de ce tissu, et nous lui arons donné un nom.

Enfin, nous venons de voir qu’il est plus généralement répandu qu'on ne le pense et qu'il existe sous diverses formes dans plusieurs groupes de Bryozoaires chez lesquels on ne l'arait pas signalé, parce que là sa forme n’a rien qui rappelle un système nerveux.

Maintenant que nous l'avons recomm et étudié dans foutes les familles, nous sommes à mème d'en donner une définition plus préeise.

Pour nous une loge de Bryozoaire considérée au point de rue abstrait, qu'clle soit zoécie ou article de tige, est composée de trois couches constitutives, savoir : l'ectocyste, l'endocyste et l'endosarrue.

1 Cisrt Vout, Aich zool. exp., t. V. 
L'ectocyste est me membrane chitincuse on encroùtée de calcaire, anhiste dans tous les eas et sur lantulle je n’ai pass à insister. Co $11^{\circ}$ 'st pas un tissu, ce n'est pas nou plus un produit de sćcrétion, e’est une conche de l'endocyste qui s'est diflérenciée el consolidée et qui n’existe même pas ehez la larve pendant la période d'activité.

l'endocyste et l'endosirnue sont au contraire les parties virantes, assentielles et fondamentales du Bryozoaire.

L'endocyste est une membrane cellulaire, un épithélium; primitirement, dans les parties jeunes et actives de la colonie elle ressemble à un épithélium eylindrique; dans les Pédicellines elle garde partout plus ou moins longtemps cette structure, mais dans la généralité des Bryozoaires marins, elle la perd de bonne heure et se réduit à une conche de protoplasme amorphe dans lequel il devient impossible de reconnaitre aucune cellule.

C'est done senlement aux extrémités régétatires que l'endocyste est organisé, et, en effet, il est spécialement chargé de l'aceroissement de la colonie.

L'endosarque dérive de l'endoeyste par différenciation des cellules des extrémités végétatives; il conserve avec lui de nombreux rapports; cependant il possède une structure distincte et est généralement composé de cellules fusiformes, ordinairement sans noyau net présentant souvent des prolongements et passant parfois à la forme étoilée.

C'est à lui qu’appartiennent toutes les formations qu'on désigne sous les noms de système nerveux colonial, de funicule, de couche fusiforme de l'endocyste.

C'est lui qui constitue la tunique musculaire des loges des Bryozoaires d'eall douce, le parenchyme des tiges et des stolons des Pédicellines et du pied des Loxosomes.

Dans son sein se produisent toujours les Zoospermes et très-fréquemment, peut-être constamment les œufs. C'est à ses dépens pour une part, peut-être exclusirement, que se forme le Polypide.

Pour toutes ces raisons, je crois qu il y a lieu de le regarder comme quelique chose de plus qu'une couche particulière de l'endocyste et bien comme un tissu spécial ayant un ròle, une structure, une position bien définis et méritaut d'ètre distingué par un nom à lui propre.

Par opposition au terme endocyste, je lui ai donné le nom d'endosarque, parce ce que nom ne préjuge rien, il a l'arantigge de n’assi- 
miler ce lissu à aucun autre aree lequel son homologie ne serait pas encore suffisamment établie, soit dans une autre elasse d'animanx, soit dims l'embryon. C'est tone un nom prorisoire qui me parait bon pour le moment el que je serai tout disposé à changer pour un autre plus général sitôl que j’aurai vu ou 'qu’on m’aura monlré son homologie avec l'ectoderme on l'entoderme des anintaux voisins ou de l'embryon. Je continuerai donc à l’employer dans la suile de ce mémoire.

\section{CIIAPITRE HI.}

REPRODUCTION PAR VOIE SEXUÉE.

\section{\$1. Origine des éléments reproducteurs.}

Nous avons ru dans un précédent chapitre que, dans plusieurs espèces appartenant à différents groupes, les ceufs et les Zoospermes naissent aux dépens du funicule, et pour appuyer nos conclusions nous avons dù domer du node de formation des éléments reproducteurs dans la Valkeria cuscuta une description assez complète qui nous dispense d'entrer dans de noureaux détails.

Dans la Bowerbankia imbricata les faits se passent de même; dès que le Polypide commence à s'ébaucher les cellules mères apparaissent dans la substance du funicule et se multiplient très-rapidement ${ }^{1}$.

Dans la Bicellaria ciliata ces cellules se montrent également de très-bonne henre dans le sein du même cordon. Elles s'en détachent par la suite et s'agitent dans le liquide ambiant poussées de côté et d'autre par les battements de la queue du Zoosperme qui en sort graduellement.

Dans lic Lepralia Martyi la masse des cellules mères est volumineuse, elle masque complétement le funicule dont elle occupe la place, les cellules s'en détachent comme dans les espèces précédentes el les Spermatozoüdes s'y forment et en sortent de la même manière.

Nous n'avons jusqu'iei parlé des œufs que pour montrer qu'ils prenaient naissance dans le sein dn funieule, mais nous n'avons pas insisté assez sur l'intérèt qu'il y a à les roir naître eôte à côte arec les Zoospermes et aux dépens du mène tissu. Ce rapprochement origi-

: P. X, fig. 3. 
naire est cependant mo lait incontestable of rien n'est plus facile que de le constater lant rlans la Bomerbantia imbricata que dans la Vallieria cuscuta, lis Birellaria ciliata el la Lagancula repens.

Dans les deux premières espèces l'ovarre se présente d'abord sous la forme d'une ecllule unique, silnée toul an sommel du limicule ${ }^{2}$, dans cette région de l'orentue ani est appliqués rontre les parois latérales du coecum stomateal. C’ost dans l’inlérienu de cefte ecllule primilive que se produisent les rleux aufs.

Le mode de formation pauait étre le mème pour l'aruf pariétal de la Bicellaria aussi bien que pour l'urnf frniculare de la mème espèce et des Bugules.

Dans la Lepralia Martyi, Ja formation de l'oeuf que nous avons déjà indiquée se peut suive avee une grande netteté.

On roit tris-bien la cellule primitive on oraire, sourent aceompaznée d’une ou deux autres plus pelites, grandir jusquà présenter l'apparence d'une grosse masse oroüde granuleuse dans laruelle se creuse une eavité ${ }^{2}$ où apparaissent deux reufs, qui mùrissent l'un appos liautre en se nourrissant de la substance de la cellule orarienne. Ouand les deux premiers wufs unt puillé la loge, un second ovaire entre en fonctions et prend la place du premier, de telle sorte yuon distingue sourent un ouf dans l'ovicelle et deux en train de mùrir dians la loge.

(in voit par là que le morle de formation des roufs est assez mniforme, el quand on le rapproche de celui des Zoospermes qui se développent à côté, il me semble qu’on peut établir un parallélisme assez complet.

L’ovaire me paraît comparable à la cellule mìe des Zoospermes. 11 se déreloppe dans les mèmes eonditions et dans le mème tissu à tel point qu'au début, dans la Ialleria cuscuta, il est difficile de distinguer l'un de l'antre.

Dans l'une il se dérelopje un ou deux Zoospermes, dans l'autre toujours deux coufs, dont l'un peut s'atrophier.

Enfin si l'on considère quue daus certaines espèces, telles quue la Lepratia Martyi, on peut trouver deux on trois de ces cellules mères d’oufs que j'ai appelées des oraires, on se rendra eompte que e'est plutôt à l'ensemble de ces paires d'œufs cu'on doit donner le nom. 
d'ovaire, comme on donne celui de testicule à l'ensemble des cellules mères de Zoospermes, et qu'il n'y a en effet de différence entre ces organes que dans le nombre des éléments, qui est toujours considérable dans le testicule et réduit à trois, deux ou un dans l'ovaire.

\section{§. Hermaphrodisme.}

Nous avons ru par ce qui précède que l'hermaphrodisme est la règle chez plusieurs Bryozoaires; c'est, en effet, le cas général, et dans presque toutes les espèces que j’ai pu étudier à ce point de vue, j’ai vu les deux éléments générateurs se produire dans la mème zoécie.

Est-ce à dire qu'il n'y ait pas d'exception à cette loi ?

Nordmann ${ }^{1}$ a décrit depuis longtemps déjà des loges mâles et des loges femelles dans le Tendra zostericola. Repiachoff ${ }^{2}$, qui a, dans ces dernières années, repris l'étude de celle intéressante espèce, a moditié les résultats de Nordmann; mais il est arrivé à cette conclusion que, si dans une colonie quelques loges étaient hermaphrodites, le plus grand nombre élaient unisexuées.

La Lepralia Martyi me parait être unisexuée. Je n'ai jamais ru aucune loge contenir à la fois des cufs et des Zoospermes, et la disposition même des parties me semble incompatible avec les nécessités de l'hermaphrodisme.

En effet, lả glande génitale, au lien d'être, comme dans les autres espèces, limitée à une certaine région du funicule, paraît l'occuper tout entier et s'étendre depuis les parois de la loge jusqua au Polypide. En second lieu l'ovaire, qui apparaît de très-bonne heure dans les jeunes loges et arant qu'aucun testicule ait pu déjà se constituer, fonctionne très-longtemps, puisqu'il peut produire jusqu’à six aufs pendaut un temps qui est à peu près celui de la vie de la zoécie.

Enfin jai toujours vu les loges dans lesquelles se développe un ovaire surmontées de très-bonne heure par un ovicelle, tandis que celles qui produisent les Zoospermes en sont dépourvues même à l’àge adulte.

\footnotetext{
1 Nordian, Voyage de Demidoff.

2 Repiachofe, Zeitschrift, t. XXY, p. 129.
} 


\section{\$3. La fécondution est récipronue.}

Ouoi qu'il en soit de ees deux cas exceptionnels, l'hermaphrodisme est la règle générale chez les Bryozoaires, en ce sens que dans cluaque loge se déreloppent ì la fois des aufs el des Zoospermes.

Mais i'hermaphodisme est-il complet, et les Zoospermes sont-ils aptes à féconder les oul's qui se déreloppent à leurs côtés dans la même loge?

C'est là l’opinion généralement reçue et cependant, en ce qui concerne quelques espèces, je suis à mène de démontrer le contraire. J'ai mème les plus fortes raisons de croire que la nécessité de lintervention de Zoospermes appartenant à d'autres loges est nécessaire au développement de l'œuf' dans la généralité des Bryozoaires.

Prenons pour premier exemple la Valkeria cuscuia. Tous arons ru déjà rque les cufs se formaient au sommet, les Zoospermes à la base du funicule.

Le tissu du testicule a déjà pris tout son développement et les cellules mères commencent déjà à se délacher, la cellule ovarienne n'est encore reconnaissable que par sa taille et sa forme un peu ovale.

Lorsque les Zoospermes commencent à nager autour du Polypide devenu adulte, c'cst à peine si l’on reconnaît dans l'ovaire une ligne qui sépare les deux aufs ; enfin quand toutes les cellules mères étant crevées, la zoécie cst toute remplie de Spermatozoïdes qui fouruillent en si grand nombre qu'ils lui donnent une teinte grise, on distingue nettement les deux rufs, mais ils ne sont encore qu'ébauchés et sont dépourvus de vésicule et de tache germinative ${ }^{\text {. }}$

Cependant chaque fois que le Polypide épanoui rentre brusquement dans sa loge, on roit un faisceau de Zoospermes sortir de l'ourerture de celle-ci et se répandre dans l'eau ambianie où ils vivent à merveille.

C'est au travers du tissu mince et délicat de la gaîne tentaculaire qu'ils passent, probablement poussés par la pression que subit le liquide cavitaire an moment de la rétraction brusque du Polypide.

Au bout dun ou deux jonrs pendant lesquels le Polypide s'est épanoui el rélracté fréq̨uemment, il ne reste plus dans la loge qu'un petit nombre de Zoospermes disséminés dans le liquide cavitaire; cependant les cufs ne sont pas encore mùr's.

1 Pl. X, fig. 2. 
Le Polypide rommence alors à se résorber suivant le mode habituel et à passer à l'état de corps brun; tous ses organes se flétrissent et s'atfaissent, se rédnisent de plus en plus; il se transforme en un véritable eorps brun, qui demenre dans le fond de la loge attaché encore an funicule par l'internédiaire duquel les denx oufs se trouvent fixés sur sa surface. Il n'y a plus ì ce moment ancun Spermatozoüde dans la zoécie; la loge est, par suite de la résorption de son habitant, complétement close; et cependant les denx reufs, qui sont encore à peu près de mème taille et renfermés dans leur enveloppe commune, présentent encore nettement leur vésicule et leur tache germinative'. A cette époque l'un des oufs commence ordinairement à prendre un développement plus grand (que l'autre, qui, comprimé par son aîné, linit au contraire par s'atrophier. L'ouf unique grandit alors à l'aise et achève de mùrir dans la zoécie hermétiquement fermée, à l'abri des Zoospermes. Pendant cette période il double et triple de rolume, devient granuleux et acquiert une teinie jame brmatre qui a causé la méprise de Lóven, qui l'a pris pour un corps brun.

A la maturité il se présente sous la forme d'une sphère assez volumineuse, jaune, finement et irrégulièrement granuleuse, présentant une vésicule germinalive très-nelte et renfermée dans une enveloppe devenue très-mince et qui présente en arrière comme un petit noyau, dernier vestige de l'œuf atrophié ${ }^{2}$.

A re moment, en un point de la paroi de la loge sur lequel sattache quelque branche dı funicule, apparaîl un bourgeon qui se développe rapidement en un petit Polypide ${ }^{3}$. Celui-ci gagne le sommet de la loge, y développe une nourelle série de muscles pariétovaginaux en mème temps que deux grands rétracteurs, mais n'atteint jamais lui-même son déreloppement complet. Les bras restent à l'état de bourgeons, l’œsophage ne se creuse jamais d'une carité et finit par se réduire à un fil, le rectum contient un méconium. Par l’intermédiaire de la branche du funicule sur laquelle il s’est léveloppé, le Polypide est en relation avec l'œuf, de sorte que la gaine tentaculaire, le Polypide, son funicule et l'cuf sont alignés sur un même cordon.

La portion du funicule comprise entre le Polypide et l'œuf se rac-

1 Pl. XIII, fig. $\ddot{3}$.

2 Pl. Xlll, fig. 8 or.

${ }^{3}$ Pl. XIII, fig. 3. 
courcit alors beancoup, tandis que la gaine tenticulaire s'allonge, le Polypide arrive ainsi à toucher l'ruff, il glisse alor's sur ses côtés et le même Polypide que javais vu le 18 juilled dans la même loge andessus de l'ouf était le lendemain passóe au-dessous, tandis que l'ouf arait pris place dans sa gaine lentaculaire ${ }^{1}$. Quelque étonnant que ce fait puisse paraitre, je laii olsservé maintes lois et le donne comme positif.

Le petit Polýpide satrophio de plus en plus, si bien qu'il ne reste plus ale lui derrière l'wnl' que son méconium²; cependant il at prèté à l'ouf ses muscles parićto-raginanx el grands rétracteurs ${ }^{3}$, ceux-ci sont insérés atu-dessous de l'œuf sur l'enveloppe qui le soutient et rui est le résultat hétérogène de la fusion du petit Polyjuide avec la gaine tentaculaire.

Dès lors, par l'intermédiaire de eette gaine l'euf est en libre communication arec l'eau ambiante, par le jen de ses muscles il peut être porté jusqu’à l'entrée de la loge, et e’est évidemment ainsi qu'il est fécondé. Je n'ai pas été témoin de l’acte même de la fécondation les tissus de la loge, de la gaine, les muscles qui masquent plus on moins l'ieuf rendraient l'observation très-difficile; mais j'ai vu trèsfréquemment les Zoospermes nager autour des loges et arrirer jusqu’à l'entrée. Je puis d'ailleurs placer le moment où la fécondation s'effectue entre des limites très-ćtroites. J'ai, en effet, vu les coufs tant qu’ils sont encore au-tessous du Polypide auxiliaire être toujours pourvus de leur résicule germinative; j’en ai observé un qui était passé au-dessus depuis quelques heures seulement et qui n'était pas eneore segmenté; un troisième, fout au début de la segmentation, était depuis peu dans la gaine, car le Polypide auxiliaire ćtait encore reconnaissable au-rlessons; enfin toutes les larves, depuis le commencement de la segmentation jusqu'ì celui où elles sont évacuées, sont toujours dans la gaine tentaculaire, et an-dessons d'elles on reconnait toujours le méconium du Polypide auxiliaire.

C'est done toujours après le moment où il a passé au-dlessus du Polỵpide que l'œuf est fécondé, e'est-ì-dire lorsqu'il est en communication avec l'eaı ambiante chargée de Spermatozoïdes.

Lorscue la larve est dans la gaine tentaculaire, elle se comporte

1 Pl. XIII, fig. 6 et 7.

2 Pl. XIII, fig. $8, m$.

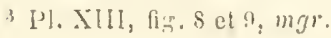


absolument comme ferait un Polypide. Attachée par son funicule au fond de la loge, mue en haut ou en bas par ses muscles rétracteurs, parićtaux et pariéto-raginaux, elle peut se retirer tout au fond de la loge ou se porter tout à l'entríe en suirant la gaine qui se déroule; c'est même par celte voie qu'elle s'échappe au moment de l'éclosion; mais l'œuf suit les mêmes mouvements avant la fécondation, et e'est sans doute au moment où il se trouve vers le haut de la loge quil reçoit le Zoosperme.

Quoi qu'il en soit, deux faits ressortent incontestablement de ces observations.

En premier lieu l'œuf de la Valkeria cuscuta n'est point fécondé par les Zoospermes développés dans la même loge que lui. En second lieu, il ne peut ètre fécondé que par les Zoospermes provenant des autres loges et qui sont constamment répandus dans l'eau ambiante.

Les faits que je viens d'exposer ne sont pas particuliers à la Valkeria cuscuta, ils se passent exactement de la même manière dans la Bomerbankiu imbricata et dans la Lagenella repens.

J'ai observé à plusieur's reprises dans ces deux espèces la larve se développant dans la gaîne tentaculaire, remuée par ses museles, portée jusqu'à l'entrée de la zoécie, et c’est assurément par cette voie que l’embryon est évacué.

Chez les Bugula avicularia et flabellata, dans la Bicellaria ciliata, j'ai vu des aufs encore pourvus de leur vésicule el de leur tache germinative dans des loges dont tous les Spermatozoïdes avaient déjà disparu. Il est done certain pour moi que dans ces espèces le concours de Zoospermes ćirangers est nécessaire à l'œuf. Reste à déterminer le moment de la fécondation.

Bien sourent j’ai été témoin de l'évacuation des Zoospermes qui lans ces espèces se fait, comme dans les Vésiculaires, à travers la gaine tentaculaire; bien souvent j'ai vu les éléments mâles nager autour des loges et les oricelles; ils peuvent done très-bien, à travers l'eau ambiante aller d'une loge à l'autre.

Une autre considération me porte à croire qu’il en est de même chez la gónéralité des Bryozoaires, c’est l'abondance des Zoospermes.

11 s'en produit habituellement dans une loge un si grand nombre que celle-ci en est obscurcie. Une telle quantité serait-clle nécessaire pour féconder les wufs contenus dans la même zoécie et qui en sont environnés de toutes parts, el n'est-il pas plus probable que, s'il se fait une si grande production de Zoospermes, e'est qu'il s'en fait une 
Erande perte et qu'un graud nombre, entraînés par les courants, ñarriveront pas à destination?

Enfin, puisqu’il existe des Bryozoailes a loges mnisexuées, il faut bien que les Zoospermes passent des loges males aux loges fomelles; par oì se fait le transport? Sans aucun doute à travers l'uau ambiante.

Cherchons maintenant s’il n’est pas possible de préciser le moment où se fait la fécondation; mais, pour cela, il nous faut aborder une nourelle question qui a fat l'objet de beaucoup de controverses, celle des oricelles.

\section{\$. Puestion des ovicelles.}

L.es ovicelles sont des cellules globuleuses qui surmontent les loges avec la cavité desquelles elles sont en rapport de diverses manières et dans lesruelles on rencontre chez beaucoup de Cheilostomes des larves en roie de développement. Nitsche a fort soignunsement décrit leur mode de formation dans la Bicellaria ciliata; mais leur forme et leurs rapports avec la loge ne sont pas partout les mèmes.

Teujours est-il que Huxley affirme que les oufs naissent dans les zoécies et ne passent dans les ovicelles que lorsqu’ils sont mùrs, pour y suive leur développement embryonnaire comne dans une sorte de uarsupium. Hincks prétend au contraire gue les œufs naissent et se développent dans l’ovicelle.

Je n’ai jamais rien vu qui puisse justifier cette dernière manière de roir, et tous les faits sont, au contraire, en faveur de la théorie de Huxley.

Nous avons vu à plusieurs reprises quel était le lieu précis du développement des cufs, nous savons que e’est la zoécie, et dans la zoécie le funicule. Nous arous ru l’œuf grandir, se détacher du lissu produeteur et se rapprocher de l'ourer ture de la loge; l'ovicelle est encore vide: à côté on trouve des ovicelles dans lesquels des oeufs, tout semblables à ces derniers, sont déjà au second stade de la segmentalion. On peut conclure de là, avec grandes chances de ne pas se tromper, que l'oeuf passe de la zoécie dans lovicelle, et que e’est à ce moment qüil est lécondé.

Je n’ai jamais assisté au passage dans les hugules ou la Bicellama, mais j’ai été témoin au moins des flemières phases de ce phénomène dans la Lepralia Martyi. 
Dans cette espèce l'ovicelle surmonte la loge, les cavités de l'un et de l'autre sont en communication par' un large canal, et la lèrre operculaire, se tromrant sur la ligne de soudure de l'oricelle avec la loge, sert ì former l'une et l'autre ì la fois.

Je vis le 4 aoùt, à huit heures du matin, un œuf qui avait déjà pertu sa tache germinative el avait abandonné l'ovaire pour gagner le sommel de la loge; peu de temps après son bord supérieur avait dépassé un peu la ligne de charnière de la lève opereulaire; à cing

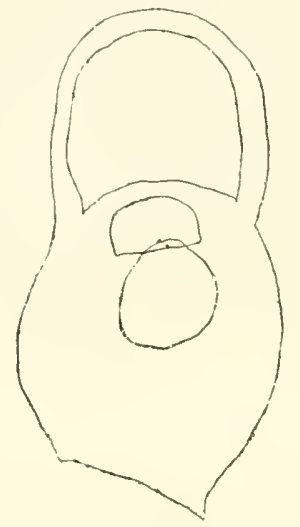

Fig. 3. - Lepralia Martyi. OEnf́ commenernt à s'approcher de l'entrée de l'ovicelle.

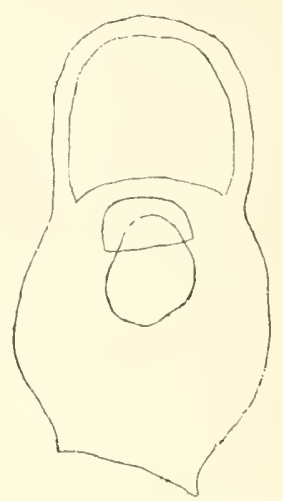

Frg. 4. - Nlime loge, dessinée à la chambre chire quelques heures après et dans laquelle l'auf est déjà furtement engagé dans le passage de l'ovicelle.

henres du soir il arait presque atteint la ligne courbe supérieure de cette mème lèvre; l'œuf était done fortement engagé dans le canal de conmunication. Je n'ai pu suivre pliss arant le phénomène, les condjLions auxquelles j'étais obligé de soumettre mon sujet pour permettre l'observation ì tout moment ayant fini par le tuer; mais il n'avait qu’à se poursuirre pour amener en quelques heures l'œuf entier dans l'oricelle.

Fussé-je même privé de cette observation directe, les faits suirants me semblent suffisants pour établir le fait de la migration de l'wuf. Si à la fin de juillet on examine un rameau bien développé de Bugula avicularia on flabellata, on reconnaît : au sommet, des ovicelles en voie

- Dans la Bicellaria et les Bugules l'œuf est aceompagné d'un bourrelet particulier' et souvent coloré de l'endoeyste de l'ovicelle, qui semble le séparer de l'entréc; ce bourrelet, qui développe comme une sorte de eaduque, a pu faire croire à Hincks que l'cult se prodnisait derrière lui dans l'oticelle. 
de formation, puis des oricelles contenant des larves ${ }^{1}$, puis une zone d'oricelles rides, puis une nouvelle région où les larves s’y trouvent; si l'on compare l'état des loges à celui des oricelles, on s'aperęoit que les loges surmuntées par des oricelles rides contiennent elles-mêmes des arufs presque mùrs, tandis que dans les autres les a'ufs, s'il y en a, sont encore ì l'étal d’óbiunclie.

11 ressort de lì nettement que dans les espèces où plusienr's oufs se forment suceessivement dans les loges, ils se suceident dans les oricelles, et que, lorsqu une larve vient d'éclore, l'ovicelle reste vide jusqu’à ce qu'un nouvel neuf rienne remplacer l'ancien.

De l'ensemble des observations et des arguments que je riens de présenter je crois pouroir tirer les conclusions suivantes :

$1^{\circ}$ Règle générale, les aufs ne peurent être fécondés que par des Spermatozoïdes étrangers à la loge qui les produit ;

@o Dans certaines espèces la fécondation par un Zoosperme étranger a lieu dans la loge même que l'œuf occupe. Ailleurs elle a lieu soit dans l'oricelle, soit au moment du passage dans l'ovicelle.

\section{§ ร. Développement de l'ouf.}

Nous arons parlé déjà de l'origine des éléments reproducteurs et de la fécondation. Je devrais maintenant traiter la question du dévelop-

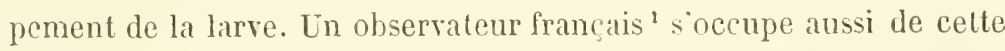
question sur laquelle il a fait paraìtre plusieurs noies. Il est nécessaire d'attendre la publication complète du travail; car les données qui ont été publiées dans des notes succinctes, ne sont pas suffisantes pour qu'on puisse se lirrer sur leur compte à un examen critique. Je passerai done celle question sous silence et j’aborde immédiatement l'étude de la fixation de la larve et de son développement en colonie.

\section{\$ 6. Métamorphose de la larve.}

Depuis plusieurs années on sait que Ies larres ciliées des Bryozoaires qui possèdent pendant leur vie errante une organisation assez élerée la perdent complétement lorsqu’elles se fixent avant de donner naissance à une nourelle colonie.

I. B. Earrois. 
J'ai pu réunir quelques observations assez complètes qui ne laissent aucun donte à ce sujet.

Les larves du Sarcochitum polyoum sont extrêmement abondantes a la fin de mai; on peut se faire une idée de leur forme en les comparant ì un champignon dont le pied serait très-court et presque aussi large que le chapeau.

Si l'on examine la larve par la face qui correspond au-dessus du chapean, on voit que le disque est bordé d’une simple rangée de grandes cellules cubiques toutes hérissées de cils vibratiles. Au centre on distingue une masse grise granuleuse volumineuse qui ne laisse entre sa surface et la bordure de cellules qu’un étroit espace occupé par un protoplasme clair, mais rempli de cellules rameuses de formes diverses.

Cette masse grise centrale montre à peu près la forme d'un cœur de cartes à jouer. Au-dessus de l'échancrure de ce cour et dans l'espace qui la sépare de la bordure de cellules ciliées, fse troure un corps ovoïde grisâtre formé de cellules olivaires disposées transversalement de part et d'autre, d'une dépression on fente longitudinale qui paraî̀ être l'orifice buceal, et est armé de longs cils vibratiles constamment en mouvement.

Ce corps celluleux est très-mobile; il peut, en repoussant devant lui la portion de la bordure de cellules ciliaires qui l'avoisine, produire à la surface de l'embryon une sorte de rostre, et l'on voit trèsfréquemment cette saillie s'aceuser nettement.

Grâce à ses longs cils vibratiles, la larve s'agite vivement dans l'eau, en tournoyant de ce mourement particulier à un si grand nombre de Bryozoaires.

Au bout de douze, quinze, vingt-quatre heures de vie active, habituellement la lirre commence à se fixer.

Ses mourements deriennent plus lents; les eils disparaissent peu à pen et leur mode de destruction est aussi facile qu’intéressant à suive sous le mieroscope. A l'extrémilé des cils qui se ralentissent, on voit d'abord perler une gouttelette réfringérente, qui augmente de plus en plus de volume en absorbant la substance du cil jusqu’à ce qu’elle soit arrivée à la surface de la cellule. Le cil se résout donc de l'extrémité à la base en une gouttclette de protoplasme. C'est là le premier indice de décrépitude chez la larve, qui reste au fond de la cuvelte el ne larde pas à s'y coller. Les cellules de la bordure ciliaire se désagrégent alors, perdent leur noyau, puis leur contour, el finis- 
sent par passer à létat de granules un peu plus volumineux que les autres el disséminés dans le protoplasme sous-jicent. Les cellules olivaires du corps ovoüde subissent le mène sort; la masse centrale grise cesse d'ètre distincte du reste de l'animal, dans lequel on ne reconnaît plus qu'une masse homogène de protoplasme granuleux.

Cette masse ne reste pas sphérique; elle s'étale sur le fond qui le supporte el y prend une forme irrégulière.

Etant parvenu à faire fixer un certain nombre de larves sur des lamelles de rerre mince que j’arais déposées au fond de mes cuvettes, j’ai pu suivre sur un même indivilu successivement toutes les phases du déreloppenent pendant une semaine.

Le 18 mai, un embryon cilié, semblable à celui que je viens de décrire, se fixa sur une de mes lamelles rers eing heures du soir.

Le 19, à sept heures du matin, il sétait transformé en une masse déprimée et à contour irrégulier de sareode granuleux, an sein duquel on distinguait nottement une sorte de biscuit de protoplasme transparent et homogène, ébauche de l'embryon.

A six henres du soir, l'embryon avait grandi et une fente longitudinale, qui se voyait déjà le matin, s'était accusée, formant une sorte de ville limité par le protoplasme.

Le 20, à six heures du soir, cet anmean s'était divisé en un certain nombre de lobes ébauches des bras.

Le 21 , à huit heures du matin, les lobes araient grandi et formaient une véritable couronne. Au-dessous se royait l'ébauclıe d'une cavité digestive, et au-dessus une sorte de cloche, premier indice de la gâne tentaculaire. De plus, une fine ligne transversale arait séparé de la zoécie primitive un segment supérieur destiné à devenir une nouvelle loge.

Le 292, à huit heures du matin, on distinguait dans le Polypicle l'cesophage, l’intestin, et même les muscles grands rétracteurs.

Dans la nouvelle zoécie détachée la reille apparaissail l'ébauche d'un Polypide.

Le 23 mai, à neuf heures et demie du matin, le premier Polypide est plus avancé ; le bord antérieur de la zoécie qu’il oecupe, qui élait droit au moment de la prouluction de lit nouvelle loge, s'est arrondi et percé d'une ouverture.

Dans le second Polypide, la couronne aux dépens de laquelle se formeront les bras est devenue distincte; enfin, sur les côtés, entre 
l'ancienne et la nourelle zoécie, apparaissent deux loges latérales qui se séparent par tes cloisons de la zoécie primitive.

Le 21 , ì tne heure de l'après-midi, le premier Polypide s'est paracluevé dans le second apparaissent les lobes rudiments des bras; enfin, sur les côtés, dans les loges latérales, deux noyaux elairs annoncent la formation te Polypides.

Le mème jour, à trois heures de l'après-midi, j’assiste au premier épanouissement du Polỵpide, qui, après plusieurs tentatives, finit par forcer l'entrée de la loge et ourrir dans l'eau sa couronne de dix-huit tentacules ciliés, recueillant de la nourriture pendant plus d'une heure.

Le 29, ce même Polypide commençait à se flétrir, les trois autres continuaient à se térclopper.

L'Alyonidium hispidum de Smitt (Flustrella hispida de Redfern) suit un déceloppement tout à fait analogue, mais la larve est douée d'une organisation plus complète et tout à fait comparable au Gyphonautes.

Entre deux valres transparentes, qui rappellent pour la forme celles d'un eypris, se trowve un tissu délicat et transparent dont la texture est loin d'ètre la même sur tous les points ${ }^{1}$. Ters la partie postérieure se frourent en effet des traînées fortement granulenses, tandis gu'en arant une petite masse de tissu cellnleux foncé, dont le profil ressemble raguement à une lête d'oiseau ${ }^{2}$, s'agite fréquemment, mue qu'clle est par plusieurstibres musculaires ${ }^{3}$ très-distinctes, qui s'attachent en arrière. Du côté tu dos de l'animal, e'est-ì-dire ver's la jonction des valves, se roit une petite saillie "portant des soies roides et qui ressemble à celle des larves de Pédicellines; enfin, du côté opposé, sur la face rentrale, les tissus bordent les valves en tedans comme le manteau d'un acéphale, et leus bord libre, constitué par le grandes cellules, est convert de eils vibratiles. On remarque une houppe de cils plus actils au niveau du corps en tète d'oiseau.

Cette larre, si compliquée aree ses cellules vibratiles, son corps mobile, ses museles, se romporte absolument comme celle da Sarcochitum polyoum. Toute la matière rirante se réduit dans l'espace de quelques heures en une masse ovoüde de protoplasme amorphe

1 Pl. XIII, fig. 3.

2 Pl. XIll, fig. 3 p.

3 Pl. Xlll, fig. $3 \mathrm{~m}$.

4 Pl. Xill, fig. 3 cs. 
fui s’amasse dans l’intérient des valres à cheval sur la charnière, of

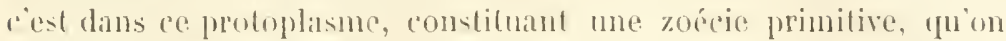

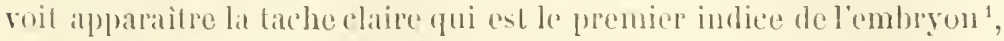
ol pue, plus tard, on voit se développer les loras et s’acherer l'orgánisation du Polypide, absolument comme dans le Sarcochitum polyoum que j’ai pris pour type, parce que ses larves sont extrêmement aboudiutes, moins délicates que celles de la Flustrella hispida, et supportent mieux l'observation continue.

J'ai observé encore avec détail le développement le la Bugulu flubellata, mais Nitsche l'a si bien décril que je ne puis gue l'envoyer à sil description, dont j’ai pu de tunt point rérifier l'exactilude².

On croit généralement que la première loge produite par la larve des Vósiculaires est une portion de la tige, dans laquelle aucun Polypide ne se déreloppe, mais sur laquelle bourgeonnent des zoécies. Il n’en est rien. J'ai suivi aree soin les métanorphoses do la larve de la Bouerbankia imbricata, et voici ce qui est résulté de mon examen :

La larve de la Bowerbantia a puelque peu la forme d'un melon, comme l'a justement fait remarquer $\mathrm{M}$. Barrois; clle est revêtue d'une enveloppe ciliaire générale, et sa conleur est d'un rouge brique pàle.

Lorsqu'elle se fixe, elle prend une forme arrondie irréguliore et se revêt d'une membrane. On roit alor's s'accuser à l'intérieur une masse rouge, dont la couleur devient de plus en plus foncéc et la structure plus granuleuse. Cette masse diminue peu à peu de volume et pourrait être an premier abord prise pour un corps brum. L'espace, qui grandit de plus en plus entre elle et la membrane externe de la larve, se remplit d'un protoplasme légèrement granuleux. Bientòt on y listingue un point plus clair; c'est lì que le Polypide commence à s'ébaucher dans la zoécie prinitive: le corps rouge, formé de matière nutıitive, ne dispariût que petit à petit, comme dans la première loge iles Bugules.

A l'exemple de Repiachoff ${ }^{3}$, je ne puis me défendre de compare la désnrganisation monnentanée de la larve à celle du Polýpide dans les loges ordinaires. C’est un fait bien remarquable et bien caracté-

1 Pl. Xili, fig. 4.

2 Nitsche, Zeitschrift, Band X.

3 Repiachoff, Zeilschrift, 26. 
ristique des Bryozoaires que cette sorte de rénovation de l'organisme obtenue par la destruction et la résorption des parties anciennes, et il n’est pas étonnant de trouver dans le déreloppement de la larve un exemple de ce qui se roit plus tard constamment chez l'adulte. Je suis disposé à comparer au Polypide le corps en tête d'oiseau de la Flustrella hispida. C'est avec la bordure de cellules cilićes le seul corps organisé qui soit dans la larve, car toute la masse centrale est composée de protoplasme plus ou moins granuleux. De plus, on ne peut pas roir ses mouvements de protrusion et ses rétractions brusques sans y reconnaître les allures d'un Polypide; enfin, les deux faisceaux de muscles qui causent ces mourements ne sont-ils pas analogues aux muscles grands rétracteurs?

C'est dans ce corps qu'est creusée la fente armée de longs cils qu'on appelle la bouche. Mais, dans tette espèce, elle n'est pas suivie d'un tube digestif, comme dans le Cyphonautes, et n'aboutit qu'à une dépression en cul-de-sac.

Je pense qu'on doit lui assimiler le corps celluleux et protrusible, également creusé d'une fente buccale dı Sarcochitum polyoum, aussi bien que la rosette de cellules que Nitsche décrit dans le roisinage des flagellums et de la bouche dans la larve de la Bugula flabellata ${ }^{1}$. Il est probable qu'une étude plus complète des différentes larves des Bryozoaires amènerait à trourer d'autres analogies et d'autres exemples des mêmes faits.

\section{CHAPITRE IV.}

CONGLUSIONS GÉNÉRALES.

Les observations nouvelles que nous arons présentées dans le cours de ce travail, les conclusions particulières que nous en avons tirées nous permettront-elles d'aborder les problèmes qui se posent successivement à tous les observateurs depuis cinquante ans sans qu'on en ait donné encore une solution satisfaisante?

Le Polypide est-il un individu, ou un organe, on un ensemble d'organes?

La zoécie est-elle un individu? Celui-ci consiste-t-il, au contraire, dans l'ensemble des zoécies, dans la colonie elle-mème?

1 Xitscns, Zeilsc'ıift, Band. XX', fig. 1. 
A qui, de la zoécie on du zoüde, appartiennent les fonctions reproductrices?

Quelle est la place des bryozoaires dans la classification?

Si jessaye de cuordonner et de discuter les iłlées qui ont étémises sur ces différentes questions el de les mettre en harmonic avec les olsservalions que j’ai exposées dans le eonrs de ce mémoire, si je tente culin de tirè de ret ensemble ruelques eonclusions générales, je ne dois pas oublier que je sor's iei du domaine des liaits.

C'est simplement l'opinion que je me suis faite au cours de ces études dont je rais donner l'exposé motiré.

Je ne reprendrai pas l'historique des dilférentes rues qui ont été proposées depuis leyssonel sur la nature des Bryozoaires. Ce travail a été fort bien fait par Nitsche et il est inutile d’y revenir". Je résumerai simplement les principales opinions qui ont cours aujourd'hui.

Une première théorie est due à Grant, a été adoptée par M. MilneElwards ct Ehrenberg, el est encore souteme quelquefois.

La zoécie et le Polypide ne formeraient qu un mêne individu, élément de la colonie. Le Polypide ne serait qu'un organe de respiration el de digestion, la zoćcie serait son enveloppe, son manteau.

Suirant Allmann, au contraire, la zoécie est un réritable individu, produisant par bourgeonnement intérieur le Polypide, lovaire el le testicule, qu'il considere comme trois formes nourelles d'individus, la première très-élerée, les deux dernières très-simplifiées.

Nitsche adopte la théorie d'Allmann arec des modifieations.

La zoécie est pour lui un indiridı, mais un individu sexué, car il ne consent pas à faire de l'ovaire et du testicule deux êtres distincts; ces deux organes font à ses yeux partie intégrante de la zoécie. La zoécie, à qui appartient lia reproduction sexuée, est également, d’après ce système, chargée de la reproduction asexuée : par bourgeonnement extérieur, elle produit de nourelles loges; par bourgeonnement intérieur, elle produit le Polypide, qui est, lui aussi, un individu, mais asexué, et chargé simplement des rapports arec le monde extérieur.

Tels sont sommairement les éléments de la discussion.

La théorie ancienne de Grant est certainement très-simple et trèsséduisante au premier aborl; mais, le l'aris de tous, elle n'est plus en harmonie arec les observations qui ont été faites depuis.

Conçoit-on en effel qu'm animal, une Ascilie, par exemple, puisque

1 Nitsche, Ceber die Morphologie der Bryozoen (Keitschrift, Band XXI, p. 471). 
e’est à côté des Imnieiers que les auteurs de cette époque s'aceordaient génératement à ranger les Iryozoaires, conçoit-on qu’une Ascidie perde périodiquement son tube digestif, sa branchie, ses unuseles, son système nerveux et se rédnise nomentanément à son manteau pour reproduire pen après tontes ees parties essentielles à la vie? C"est cepentant là ce qui se passerait eonstamment chez le Bryozoaire chaque fois que le Polypide vient à se résorber pour faire place à un noureau bourgeon.

D'autre part, un appareil qui comprend : tube digestif, branchie, muscles, sysième nerreux, est assurément plus qu'un organe, c'est un individu. 11 n'est pas possible de voir le Polypide sortir' de sa loge timidement, y rentrer brusquement an moindre choc, sentir, manger, respirer, se mouvoir, sans comprendre qu'il y a là plus qu'un syslème et bien un animal complet.

Enfin, ruand on étudic les Vésiculaires, on est bien foreé de s'habitner à séparer la zoćcie du Polypide, puisque dans ce groupe de Bryozoaires, il exisle un stolon composé de réritables loges, possédant tous tes caractères et la constitution des loges normales, mais dépourues constamment de Polypide.

Il y a mieux. Dans les Pédicellines non-senlement il existe un stolon comme dans les Vésiculaires, mais il se tronve des loges qui sont animées de mouvements fort énergiques, bien qu'elles ne renferment aucune trace de Polypide; les tiges des Pédicellines, entre le moment où elles viennent de laisser tomber leur tête jusqu'à celui où elles en reprement une nouvelle, sont en effet aussi contractiles qu'avant ou après celte période.

Le Polypide et la zoécic sont done denx choses tout à fait distinctes. Si l’on ne comnait pas de Polypicle sans zoécic, on voit tous les jours des zoécies sans Polypide.

La théorie d'Allmann modifiée par Nitsche en ce qu'elle a de trop excessif est plus en harmonie arec les observations récentes. Si la zoécie est un individu, il est naturel (qu'elle puisse subsister par ellemême el qu'on puisse la trourer dans les Vésiculaires aussi bien que dans les Pédicellines, indépentamment du Polypide; il est convenable aussi, si on la compare, comme l'a fait teuckart, à un kyste produisant des cysticerques, qu'elle bourgeonne des individus d'une autre forme sur sa surface interne; enfin, il n’est nullement étonnant que ces intividus, n’ayant qu'une existence éphémère se succèdent dans une même loge. 
Callo théorie, fui, comme on le roit, rend hien compte de l'existene de \%oćries sams Polypile et du penombellement lu Polypide,

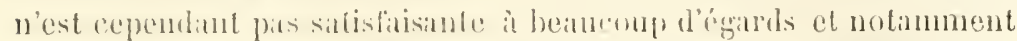
an point de rue de la reproduction ef des lomologies à élablir entre les Entoprocter el les Eetoproctes.

11 peut paraitre en eflet singulier que lat zoécie cumule lnutes les fonctions reproductrices, reproduction sexuéc cl asexuéc, reproduction des loges, repronluetion les Polypides, reproduction les larves, tandis que le Polypide, possédant une ol'ganisalion si complèle, n’a d'autre ròle que de digérer.

Puisque nous reconnaissons récllement dans le Bryozoaire deux formes d'individus, ce fait de la localisation de toutes les fonctions reproduchrices dins une seule d'entre elles est sins analogue ailleurs.

Dautre patrt, il est hien recomnu que, ehez les Pérlicellines, fue l'on considere la tête comme une zoécie complète on comme un Polypide, dans tous les cas, la reproduction sexuée apparlient à re dernier. on se tronve done obligé, ou de se meltre en désaccord arec lit théorie d'Allmann-Yitsche ou le reconnaitre entre les Lntoproctes cl les antres Bryozoaires des différences si profondes et d'un orrle si chevé, quelles obligeraient presque à placer les premiers complétement en dehors du groupe.

Or, les Entoproctes me paraissent si bien liés aux autres Brỵozoáres, qu'il y a plutôt licu de se demander', ce me semble, si on ne doit pas rapporter gínéralenent la reproduclion sexuée au Polypide plutôt qu’à la zócicie.

Je sais hien que quand on songe a l'oeuf pariétal des ficellaria se féveloppant sur un rameau de l'endosarpue au contact des parois de la loge, le rapprochement neut paraitre forcé au premier abord; mais, d'antre part, l'ouf des Vésiculaires naissant directement contre le ceccurn stomacal el même un peu au-dessus de son extrémité, l'œuf funiculaire des Bicellaires, des Bugrules et de l'tucratea se dévelop. pant immédiatement ali-rlessous de ce même creum sout bien dans la dépendance inmédiate du Polypide. Et le testicule, ne se formet-il pas toujours au scin du funicule, portion, il est rai, de l'endosarque, mais portion modifiée, appropriće aux fonctions fircelle remplit près du Polypide et véritable annexe de celui-ci, dont elle forme comne un troisième muscle rétracteur?

un mobjectera que l'éloignement entre le lolypide et les organes 
reproducteurs dans plusieur's espèces. telles que les Flustres ou les Hembranipores, rend mon opinion insoulenable. Nais sont-ee les seuls organes dans ce cas? (Uuels rapports, par exemple, ont avec le Polypide les museles pariétaux?

Ils ne sont nulle part en contact aree lui, la plupart en sont plus éloignés que ne sont leş̧organes reproducteurs, ot cependant ils se développent en mème temps que le Polypile, disparaissent avec lui et, tant qu'ils existent, restent sous sa dépendance el obéissent à sa rolonté. A quel titre les distinguerait-on des autres muscles qui tous sont et ont toujours été regardés comme appartenant au Polypide?

N'est-ce pas lì un cas tout à fait semblable à celui des ceufs?

D'ailleurs les éléments, les organes reproducteurs ne sont-ils pas liés directement dans leur développement au développement du Polypide?

Dès que s'organise le jeune bourgeon, on roit apparaître derrière lui les cellules mères des Zoospermes et des oufs; les Zoospermes ne vivent pas au-delà de sa rie, ils disparaissent arec lui ; les œufs, il est vrai, durent plus longtemps, mais est-ce le seul exemple dans la nature d'œufs survirant à la mère?

Enfin, dernier argument: a-t-on vu jamais des œufs ou des Zoospermes dans des zoécies privées de Polypide, s’en développe-t-il dans les articles de tige des Vésiculaires, s’en forme-t-il dans les zoécies avant la naissance d’un bourgeon ou après la destruction du Polypide?

\section{Pour résumer :}

Il y a de nombreuses espèces d'Ectoproctes chez lesquelles les éléments générateurs se développent dans le roisinage immédiat du corps du Polypide et dans un organe qui en dépend.

Les œufs et les Zoospermes sont toujours dans la dépendance du Polypide, en ce sens qu'ils accompagnent toujour's son déreloppement et ne se forment jamais sans lui.

Dans les cas où les ćléments générateurs se forment sur les parois de la loge et à quelque distance du Polypide, ils appartiennent à ce dernier au même titre que les muscles parriélaux.

Pour toutes ces raisons, je ne puis m'empècher d'attribuer au Polypide les fonctions reproductrices par voie sexuée.

L.es conséquences de cette manière de voir sont les suivantes:

Les Entoproctes, au lieu de constituer un type aberrant, ne sont 
qu'un tỵpe perfectinuné at d'ailleurs bien caractérisé, mais rentrant dans la loi grínériale.

Toul bipozonire complend deux sortes d'individus, la zoécie et le zoÏd( .

L.a zoćcie cst chargée de la roproduction asexuće; par bourgeonnemcnt, alle produit le zoïde.

Le zol̈de est charexé de la reproduction par voie sexuéc; il produit lit larer.

Ur, la première zoécie, souche de la colonie nouvelle, n'est pas, comme on l'a dit, engendrée par la lapre. C'est la larve elle-neeme qui se mólamorphose comme la chenille se métamorphose en chrysalide, éct-it-dire en conservant son individualité. On pent donc dire que le zoïde produit par voie sexuée el sans intermédiaire la zoćcie.

Cette snceession de phénomènes constitue une alternance de formes bien caractérisće, arec celte seule particularité cue l’individu de seconde génération reste constamment attaché ì celui sur lequel il a bourgeonné.

Les ripports qui existent entre la zoécie et le zoïde me paraissent pouroir èlre comparés très-exactement à ceux qui règnent entre l'anatife et son pied. Dans cet animal, le pied peut produire par bourgeomnement plusienrs animanx parfails, et les organes génilaux, tout en appartenant réellement à l'animal parfait, paraissent sourent se former dans le pied.

Dans la plupart des espèces, la zoécie hourgeonne successivement plusieurs Polypides stóriles, qui nont d’autre objet que de prendre de la nomriture el d'alimenter la colonie jusqu'à ce qu'il s'en produise un sexué; après quoi, la zoćcie meurt.

An contraire, chez les Pédicellines, dans la zoécie qui constitue la tête, il ne se produit jannais qu’un Polypide. A yuoi cela tient-il ? A ce que le Polỵpide des Pérlicellines est tonjours sexué. Après la reprouluclion, la zoŕcie meurt avec le zoïde, suivant la loi générale, et tombe.

Nous allons encore trouver dans cette théorie l'explication naturelle des différences qui existent entre les larves des Entoproctes et celles des Ectoproctes.

J'ai déprit phus haut, dans les lan'ves de l'd leyonidium hispidum et du Surcorhitum, nin corps que je regirde comme l'homologue d'un polypide. Ponr moi, ces litres, hautement organisées, peuvent èlre considérías comme le composé l'me zoćcie et d’un zoüde, tous deux modifiés en rue de la vie errante que doit mener la larve. 
Quand celle-ci est sur le point de se fixer, le zoïle qu'elle contenait se résont comme ferail celui d'une loge ordinaire, et, en se r'ésolvant, entraine, dans la zoécie qu il babite, des modifications correspondantes, telles que la chute des cils el de l'épithélium ciliaire.

De même, dans la larve des Pédicellines, on distingue, et même plus nettement que partout ailleurs, la zoécic et le zoüde. La zoécie, en forme de coupe, ressemble ì une tête ordinaire de Pédicelline; le zoïde remue dins sa demeure et, sauf ses deux grands lobes ciliés, se rapproche beaucoup de celui des têtes normales. Pourquoi, dans ces larves, n’y a-t-il pas désorganisation totale comme dans celles des autres Bryozoaires?

Parce que dans les Pédicellines, le zoüle, comme nous venons de le voir, ne se résout pas, ne se renouvelle pas, et que la loge suit sa destinée. Elle continue à vivre et bourgeonne directement un commencement de stolon.

Les larves des Pédiccllines sont done parfaitement comparables, à mon avis, à celles des autres Bryozoaires, et l'on peut suirre clans les différents groupes le perfoetionnement des formes:

Dans les larves de plusienrs Lepralia que j’ai examinées, je n’ai pu reconnaître l'analogue de ce coíps celluleux que j'assimile au Polypide dans le Sarcochitum et dins la Flustrella.

Dans le Sarcochitum, il semble réellement jouir d'une certaine individualité, car on le roit se contracter, exécuter des mourements indépendants de ecux de la larve. be plus, il est pourru d'une ouverture qu'on s'aceorde à nommer braccale, el cetle ourerture est garnie d'un faisceau tout spéeial de grands cils.

Dans la Flustrella hispida, ce eorps non-senlement est contractile, pourvu d'une bonche et te cils stéciaux, mais il est mu par de véritables muscles, et il ressenble certainement plus à un Polypide que le corps rond des A vieulaires, qu'on s'accorde pourtant généralement ì considérer comme l'homologne de ce dernier.

Enfin dans les Pédicellines, la coexistence dans la larve d'une zuécie et d'un zoïde n'est plus contesiable.

On voit par là que les formes larvaires les plus simples sont constituées uniquement par une zoécie, dans laquelle l’ébauche d’un Polypide est peu ou point reconnaissable, ce qui confirme encore une fois ce yue j'avangais tout à l'heure, à savoir que la zoceie est le prohuil direet de lit génération sexuée. Ciest dans la larve que la zoćeie alleint son organisation la plus élevée, parce qu'olle mène une vic 
errante: son endocyste n’est pas seulement un épithólium cylindrique et bien organisé, écst, au moins par places, mn épithélinm ciliaire "; le Polypide, an contraire, est al l'état pudimentaire et d'antant plus rudimentaire que la larve est phus actire. Les larres de Surcochitum sont moins agiles que celles des Bugules; colles de la Flustrella sont paresseuser ct se trainent au lond tes àve!tes; enfin, celles des Pédiecllines ne remuent que par bontates.

(Gue penser maintentunt de la thérie de Irackel, gui vent envisager l’individu comme représenté non pas par la zoćrie, mais par l'ensenble des zoécies par la colonie?

Sims donte, toute la colonie naìt l'une mime lare ; sams doute, dans plusicurs Bryozoaires d'ean donce, les loges ne sont pas distinctes, ce qui force à donner ì l'ensemble des êtres issus d'une même larve un certain degré d'unité. Mais, d'autre part, quand on considère rue dans les Loxosomes les zoécies, à mesure qu'elles bourgeonnent sur la zoécie primitive, s’isolent, se détachent et mènent une existence séparée, il pourra semller difficile d'envisager comme ne formant qu’un mème ìtre les zoécies dispersées gni sont issues d'un moime parent.

De phus, quand on prend en eonsiltération les formes variées que pent affecter la zoécie, comme Leuckart la si justement démontré, zoćcie, aviculaire, vibracule, épine, article de lige, fibrille radienlaire, on ne peut se défendre te regarter, comme une entité particulière, un être qui affecto des formes si variées.

La zoécie reste don: pul' nons l'une de; denx formes d'individus. dont l'union constitue le Bryozoaire.

Abordons mantenant le second des deux problèmes que j’a posés eu commençant eette diseussion.

Quelle est la plice des bryozoaires dins la classification?

A cette question, je ne puis domer actuellement aucume réponse positive. Les matériax, les ćléments que j’ai puréunir jusqu’ici sont encore insuflisants. Il fuulrait non-seulement aroir une connaissance plus approfondie que celle que j’ai pu ac puérir des formes larvaires et du déreloppement, mais eneore possíder des termes de enmpa-

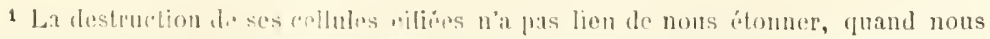
voyons dans les zoécies des colonies l'ẹpithélium cỵlindrique des extrémités régétatives se résoudre en protoplasme amorphe. Cette transformation de l'endoeyste caraetérise partout daiss la larre comate dans l'état adulte de la zoécie. 
raison précis dans les groupes auxquels on peut être tenté de les rapporter.

Je répète done que je ne puis fixer la position des Bryozoaires" mais je me sépare complétement des auteurs qui veulent avee Reichert les rapprocher des Hydraires, sous prétexte que leurs éléments histologiques sont pen définis.

Je trouve, au contraire, que les caractères des différents tissus sont aussi nettement aceusés que chez les animaux les plus élevés.

L'endocyste les extrémités végétatives dans tous les groupes et celui de toute la colonie dans les Eutoproetes présente la structure cellulaire la plus nette.

L'endosar'que a pour élément type la cellule fusiforme, qui peut se modifier et devenir rameuse.

Dans le Polypide, on distingue une grande variété d'éléments, cellules ciliées des tentaeules, cellules cilices du pharynx, cellules losangiques du pharynx des lagenella, cellules ciliées de l'estomac, cellules ciliées du rectum, enfin cellules hépatiques, car je ne puis considérer autrement que comme des éléments hépatiques les celIules à granules bruns qui fondent pendant la digestion, mèlent leur contenu aux aliments, et sont généralement répuudues chez les Bryozoaires. Un tel tissu, assurément, ne se rencontre que chez des êtres possédant une organisation déjà arancée.

Et les muscles auxquels Reichert n'accorde que le nom de corduns contrartiles (contractile Strenge), sont-ils si simples, parce que leurs fibres sont isolées? Il suffit d'examiner, dans l'Eucratea chelata, les mnseles grands rétracteurs, pour se convaincre au premier coup d'uxil, et mème sans le secour's les réactifs, qüils présentent la striation la mieux accusée.

Ce sont de réritables fibres striées, et le fait de leur contraction brusque est bien en rapport aree cette donnée.

Si de l'examen histologique on passe à celui de l'organisation en général, on n’est pas moins frappé de la supériorité de ees êtres.

Reichert ${ }^{1}$ a beau distinguer dans le Zoobutryon pellucidus le ganglion nerveux ef le figurer, il ne reut pas le désigner sous ce nom, mais il en fail me particule exerementitielle qui viendrait toujours s'aceoler à la hase des tentarules an sortir de l'amms.

Il est cepentant impossible de mécomnaître son existence dans les

1 Leicnent, Abhandhungen, $1870, \mathrm{pl}$ I, fig. 3, de. 
Entoproctes, apròs les recherches de Nibehe et de Salensky ${ }^{1}$; dans les lirvozoaires d’ean douce, il a ćlé lròs-généralement dérit; dans les ectoproctes marins, il est plus difteile à roir ; cependant, Nilsche l'a ru daus les Flustres, of jai pu constater que dans l'Eucrafer ii Bail fort bien développé.

Je ne puis done m’associer aux vues des disciples de Reichert, et pour noi un type, qui a pour représentant le plus ćlevé la Pédicelline el le Joxusome, est assurément un lype tròs-supérieur aux Colentérés.

\section{CIIAPITRE V.}

ESPĖCES RECUELLLES A ROSCOFT PENDANT LES ÉTÉS DE 1876 ET 1877.

Ce n'est point une classification que j’ai l’intention de proposer ici, èest une simple liste des espèces que jai trouvées pendant deux ćlés dans la seule localilé de Roseoff, e’est-à-dire dans une aire qui peut aroir trois lieues carrées.

On ne s’étomnela done pas si je les énonce dans un ordre qui n’est sans doute pas le meilleur, et si je donne à quelques expèces des noms qui pourront paraitre suramnés.

Pour dresser un eatalogue méthodique et systématique, il faudrat avoir entre les mains, non pas les formes d’une seule localité, mais celles des régions les plus varićes, il laudrait soumetlre à une erilique sévòre les déterminations des auteurs dans lesquelles il rỏne parfois, jai pu le constaler spécialement pour les Yésiculaires, me grande confusion; il faudrait enfin examincr et comparer un grand nombre fléchantillons atin de connaitre les modifications que peut subir un mème trpe.

C'est surtout lorsqu'on étudic la division des Cyclostomes qu'on se rend compte de la nécessité où l’on se trouve, pour bien comprendre un grroupe, d’en faire une étude toute spéciale.

Rien de plus variable, en effol, dans cetle famille que les formes gue peut affecter la mênte colonie à deux éporpues différentes. On a fail arec la même pspòce des grenres diflérents. La station particuliirement me paraîl avoir str le développement de ces cires une in-

1 Nitscie, Beilrage (Zeitschrift, 1. XX). - Salexsky, Sur les Bryozoaires entoproctes (Ann. sc. nat, 6e sirie, t. V, p. 11). - Nitsche. Beilrage (Zeilschr, t. XXI). 
fluence notable; tel Tubulipore virant sur les algues filamenteuses n’est peut êlre pas différent de tel autre qui se trouve plus spécialement sur les larges frondes des laminaires, ou sur les expansions minees des ulves, et aurquel celte station fait prendre une physionomic prarticulière.

Toutes ces modifications et variations peurent faire l'objel d'une étude intéressante, mais minutieuse et surie, que je ne puis songer à aborder en ce moment.

Je me bornerai done. je le répète, à donner le eatalogue des espèces de Roseofr, et à relater les eonditions dans lesquelles je les ai rencontrées. C'est un doeument qu’il ne sera pent-ètre pass sins intérèt de comparer aree ceux gue Van beneden et M. Fiseher nous ont déjà foumis sur la fame bryozoologique des còtes d'Ostende et de la côte ouest de France.

Il y a deux moyens de recueillir les Bryozoaires, comme d'ailleurs la plupart des animax marins, moyens gui s'adressenl ordinairement à des espèces différentes et sans l'un ou l'autre descyuels la recherehe serait forcément ineonplète.

Le premier consiste à explorer la grève à pied en cherehant à tous les niveaux, sur les pierres et sous les pierres, sur les algues et parmi les zostères.

Le second, ì ramener des fonds que les eaux n'abandoment jamais ton- les corps gui les tapissent, et à examiner avec soin les pierres, les coquilles, les algues, les pieds de gorgones et les débris de touto nature que les engius ramenent au hasard.

Le premier mode est certainement le plus varié, celui qui fournit le plus grand nombre d'especes; celui aussi qui dome sur leur sta. tion, smr les conditions dans lesquelles clles vivent, les renseignements les plus précis.

Je n'ai rien l'ailleurs à en dire de bien particulier. Pour peu gu'on fonille la grère attentivement, armé d'un conteau, d'un martean et d'mu eisean pour enlever les espèces cneroutantes, d'une pince fine pour détacher sans les écraser les toulles délicates des Bugules ou autres espreres rameuses, on se fera promptement à Roscolt une collection assec étendue. Cependant il y a nombre d'espèces qui se ticmnent dans des parages nollement rireonserils, sans que rien semble en apparence moliver ee choix. On n’aequiert frò la longue la

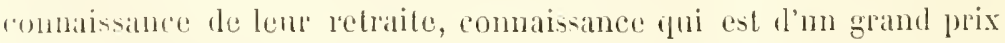
pour l'observalem qui poursuit des recherehes suivies. Bien sourent 
il mest allivé, lorsque le besoin se faisait sentir d'échantillons frais, d'aller les ehercher à coup sur et à point nommé. Il y a mieux, grlice a la connaissance que j’ai acquise des lieux, grited aux notes qui ont été prises lant sur les cartes que sur tes registres, je puis durant tont l'hiver el j’ai pu dès l’année dernière ne fanire adresser par le gardien du laboratoire lelle ou teále espece qui miarrive à la Sorbomne parfaitement virante dans les envois yue le laboratoire de zoologie expérimentale recoit régulièrement de son annexe maritime.

L'explosation des fonds demande an contraire un onlillage spécial, et éest dans celte recherche qu'on appricie les services qu* penrent rendre aux cherchenrs l'organisation, le matériel el le pelsonucl d'un laboratoire.

Suit que je fisse ilraguer spécialement pour cet objet, ayant à ma disposilion, comme tous les travailleurs athnis à Roscolf, les ambancations du laboratoire, soit que je me contentasse d'examiner les résidus des dragruages faits pour la récolte des mollusques, des ćchinodermes ou autres animanx de fond, j’ai eu, je puis le dire, pendant ces deux étés, plutôt excès de matériaux, el le plus sourent l'élude de l'organisation des types les plus intélessants ne me laissait pas le loisir d'examinel tout re qui m'était apporté.

11 est done tros-probable que jai lai-sé passer un rertain monbure d'espèces, surtout parmi les Leprolia, qui sont si dillficiles a distinguer les unes des antres an prenier abord.

Malgré ces lacunes, je suis à même, dès aujunrl'hur, de prósémler une liste de soixante-puatorze espèces recueillies uniquement à lioscoff. Ciest un chifre certainement fort élevé, el le plus élevé fưon ait présenté jusqu ici pour une seule localité.

La dlague m'a rendu de grands services, mais l'instrument qui ma procuré les meilleurs résultats pour la recherche eu eau profuncle est assurément l'engin des corailleurs.

M. de Lacaze-1)nthiers, qui arait été mieux que personne ì mème d'apprécier en Afrique les services qu’il pouritit rendre au naturaliste, l'avait à plusicurs reprises employé arec succès dans ses recherehes à la mer el importé à Roseoll dès la création du laboratoire. Denx bras de bois sont liés en eroix; aux quatre extrémités sont allathés des parguets de vieus filets, on mienx des filets de corde peu tordue. Un cinfuieme paquel plus gros gne les antres, of que les matrins appellent la quene du diable, est lixé au centre du systène en mène 
temps qu'un plomb d'un poids suffisant pour maintenir le tout au fond de l'ean sans toutefois mouiller l'embareation.

On attache une corde solide à l'engin, et on le jette par-dessus bord; on abat les voiles ot on se laisse dériver lentement an courant, traînant la machine au fond de l'eau pendant une heure, une heure el demie, jusqu'ì ce qu'on ait parcouru un espace de 300 à 400 mètres. Si le courant n'est pas trop fort, et l'on choisit pour faire cette pêche la fin du flot on du jusant, on les mortes marées, les filets s'étalent sur le fond, enveloppent les objets qui le couvent, les accrochent, les arrachent et les retiennent dans leurs mailles ainsi que dans les poches qu'on a eu soin de disposer à cet effet. On obtient par ce moyen, à coup sùr, tout ce que les pêcheurs évitent de ramasser ou ne rapportent que par aceident, tous les objets qui croissent sur les fonds lochenx dangereux pour les filets.

Quand on ramène l'engin à bord, il est ordinairement chargé d'Oursins, d'Etoiles de mer, d'Aleyons, de Gorgones, d'Ascidies, et surtout d'Eschares, de Salicornaria, de Cellepores, de coquilles et de pierres fouvent chargées de Bryozoaires, tels que Tubulipores, Bugnules, Bicellaria, Vésiculaires, Pédicellines.

Comme sur la grère, il y a dans les fonds des parages spécialement favorables à la recherche de telle ou telle espèce.

Les Eschara ceveicormis, Cellepora ramulosa et Slienei proviennent presque exclusivenent des bancs de l'Ouest, les Eschara foliacea, Serialarin semicomvolufa, Bicellaria, Lagenella mutans, Avenella fusca, surtont d'Astan et du nord de l'ìle de Bas.

Guelques espèces, ailleurs très-communes, font absolument défaut ì Roscolf; telles sont l'H leyonidium gelatinosum, les Flustra membranacea, Bugula neritina, Memipea ternata.

En revanche, j'y ai rencontré plusieur's formes qui n'avaient élé signalées que dans llatutres mers, et quelques autres me paraissent entièrement nouvelles.

La Serialaria semiconvoluta n'avait été signalée jusqu’ici, à ma connilissance, que dans la Méditerranée; j’en ai recueilli plusieurs échantillons venant des plus grandes profondeurs existant à Roseoff, cest-it-dire de 7is it 80 mitres.

La C'urbusen indivisa est regardée comme propre ì la NouvelleZćlande. 11 m’rst impossible de trouver cependant ancune différence entre les dessins que Busk donne de eette espèce et celle que jai rencontrée dans les dragnagges, et qui ne se rapporte nullement d'ail- 
laurs ì la Carbersea papyeren, la seule connue jusqu'ici sur nos côtes.

Lat lembranipora spinosa (sp. nor.) dillëro notablement de tontes les membranipores connues dans nos parages, el spécialement de lat Merbranipora pilosa dont elle se rapproche to phus.

Le Loxosomn phascolosonatum découvert par M. Lenirre, ancien préparatenr du laboratoire, puis étulié et decrit dans les frohices de Zoologie experimentale, par M. Carl Vogt ${ }^{1}$, est encore me espece propre à Roseofl, jusqu ic i du moins.

La Lagenella mutans est une fort intéressante Vésiculaire qu'il ne m'est possible de rapporter à ancune description. Je l'ai longtemps confondue aree la Laguncula reprens de Van Beneden, juscuu'à ce qu'il m’ait été domné, gràce à l'obligeance de M. Ed. Van Beneden, de pos-. séder quelques échantillons de cette dernière espèce, dont la mienne diffère par les caractères les mieux tranchés.

Enfin la Lepralia Martyi est une belle forme de Lepralia à parois transparentes dont je ne troure pas l'analogue dans les auteurs. Je me fais un plaisir de la dédier au garçon de laboratoire, au patron de barque intelligent et déroué, Charles Marty, qui m’á si constamment el si efficacement secondé dans mes recherches à la grère.

C. Vogt., Arch. zool. exp., t. V. 


\title{
CATALOGUE DES ESPECES
}

\author{
REGUEILLIES A ROSCOFF EN 1876 ET 1877.
}

\section{GYCLOSTONES.}

Tuluelipore patina (Johnss., Brit. Znophe, 18\%, p. 260). - Triscommun sur' l'Escleara foliacea.

Tubulipora hispida (Johnst., id., p. 268). - Assez fréquent sur l'Escliara folincea à Astan.

Tubulipora serpens (Jolnst., id., p. 270̈). - Commur sur les fihrilles des Algues dans l'herdier, particuliemement derant l'ile Verte.

Tubulipora flabellaris (Johnst., id., p. 274). - Sur les Uhes et sur Jes Cystosema depuis les hauts niveaux jusqua ì la zone des Sargasses.

Tubulipora diffrent des espices do Johnston, n'ost peut ètre qu'une forme du Tubulipora hispida. Je n'ose lui donner un nom noureau. Sur les pierres schistenses à Rollea-Saint-Pol.

Alecto dilatams (Johnst., Brit. Zoople., 1847, p. 281).--Sur les pierres à Rollea-Saint-Pol.

Alecto gramlata (Johnst., id., p. 280). - Sur les vieilles roquilles de pecten, de Lutraires dans les draguages à Astan et dans la baie de SaintPol.

Alecto major (Jolust., id., p. 281). - Partunt dans les draguages.

Diustopora obelia (Juhnst., id., p. 2-Ti). - Dans les draguages.

Pustuliyora deflexa (Jolnst., iil.). - Sur les pierres et les vieilles coquilles. Rollea-Saint-Pol.

Crisia denticulata (Johnst., id., p. 284). - Abondante à la face inférieure des roches, mais surtout sur les sonches du Cystoseira fibrosa, qu'elle coure de touffes épaisses. On la troure aussi, mais plus rarement, dans les draguages. Son maximum de développement est au-dessous des Laminaires, dans la zone dite des Sargasses.

Crisiu eburnéa (Johnst., p. 283). - Fréquente sur les souches de sargasses daus le chenal.

Crisia aculeata (Johnst., 1. 285). - Dans les draguages. 


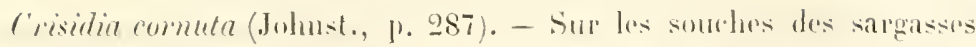
et sur divers corps sous-matrins, gorgomes, vieilles copuilles, a toules

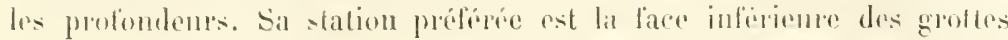

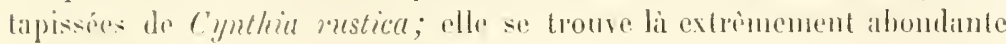
au mivean des bases mers moyenues.

\section{CHEILOSTONES.}

Scrupncellaria seruposa (Busk, Mar. Polyz., p. 28). - Allúrente aux pierres plites daus les draguages et i tres-basse me1. Rolloa, Cäuou, Dusten, Per-Roc'h. Je l'ai trouvée en reproduction au mois d’anoit.

C'anda reptans (Busk, id., p. 26). - On en troure ì Roscuff deux formes assez tranchées rorrespomlant i des stations différentes.

L'une dielles est formó de hranches griles, élancres ol qui, sopties de l'eau, s’aflaissent l'une sur l'autre. On la rencontro sous lis rochers et les grotles ì l'abri de la lumière, au niveau des basses mers moyennes.

L'autre pousse soit sur les feuilles du Baudrier de Neptume, soit sur los ramuscules terminams du Cystoseira fibrosa ${ }^{1}$, oir elle est tris-abondiute. Les branelies sout do forme plus trapue; alles sont disposées comme en ixentail et se tiennent assez roides yuand on les sort de l'eau.

Aucune différence appréciable dans la structure des loges ne distingue ces deux rariétés.

Salicomarin farciminoides (Busk, id., p. 16). - Sur les Gorgones, sur les Eschares par 30 ou 40 metres de profondeur, surtout dans l'ouest sur les basses de Roch-Haro, mais anssi à Astan, dans le nord de l'ile de Bas et du côté de ia Méloine.

Je suis complétement d'accord avec Busk pour almettre que les Saliconnaria farcminoides et simuosa de Hassall ne sunt fu'me seule et mème espèce. Sur phusieurs érhantillons al notamment sur un spérimen appartenant à la collection de Roscoff, on roit nettement la forme farciminoides se dílacher de la forme sinuosa constituant simplement une des branches de la touffe. D'autre part, je ne crois pis que l'une soit la forme adulte de l'autre, elles restent toujours distinctes sur des branches différentes. Les branches de la forme farciminoides sont plus grèles, plus longues que celles de la forme sinuosa, dont les articles sont plus courts et plus gros. J'ai toujours vu les cellules élargies vers le liaut et à ouver-

1 Les Algues ont été déterminées à l'aide du riche et bel herbier formé et laissé au laboratoire en août et septembre 1876 par IIM. Sirodot, doyen de la Faculté des scienees de Rennes, ot Gallée. 
ture subterminale te la forme sinuosa conserver leurs caracteres jusqu'it l'exlrémité des jeunes branches, aussi bien yue les cellules bosangiques des jeunes branches de la forme farciminoides gardent leur aspect jusyu'aux articles basilaires.

Eucratea chelata (Johnst., Brit. Zooph., 18.4, p. 289). - En petits faisceaux sur les fouiles de la Lammaria saccharima. Sonrent parasile sur les Bugules. Commune aux Cirejers sur les Algues filamenteuses fortes tpui peudent sous les roches (Cladophora rupestris). C'est surtout i RolleaSaint-Pol quielle est ahondaute. Elle coure de ses toufles diverses Algurs rouges. Zone des filets et des Laminaires.

Mippothoa divaricata (Busk, Mar. Polyz., p. 30). - Sur l'Ascidia sanguinolenta à Per-hoc'h. Rare.

Anguinaria spatulata (Busk, id., p. 31). - Fréquente sur les branches basses des Sargasses, sur les fruilles de Nitrphyllum et sur diverses Algues rouges à Rollea-Saint-Pol, Duslen, draguages. Cette esprece apparait au niveau des plus basses mers et descend dans la profondeur.

Beania mirabilis (Johnst., Brit. Zooph., 1847, p. 361). - Gette jolie espèce se trouve fréquemment dans les produits des draguages, sur les Cynthia glacialis, les Eschares, les touftes de Salicomaria. J'en ai rencontré à très-basse mer sur les pierres à l'ombre à Duslen et au Cä̈nou. En reproduction au milien d'août.

Caberea Hookeri (Busk, Mar. Polyz., p. 39). - Assez rare. Dans les draguages, en grandes toufles sur les vieux fragments d'Eschares.

Caberea Boryi (Busk, id., 1. 38). - Fréquente sur les Eschara foliacea; bien distincte de la Caberea Hookeri, elle a toujours ses branches disposées en érentail.

Bicellaria ciliata (Busk, id., p. 41). - Commune en petites touffes sur beaucoup d'objets provenant des draguages, sur les Cynthia glacialis, sur les branches de Vesicularia spinosa, sur les carapaces de divers Parthénopiens, sur les Eschares, sur les Gorgones et quelquefois sur la roche vive.

J'en ai trouré une belle toulfe à Per-Roe'h par une basse marée, mais ce fail at exceptionnel. La zone de la Bicellaria commence au nireau des plus basses mors et s'étond justu'nur plus grandes profondeurs existant dans les parages de Roscoff, e'est-i-dire jusqu’a $7 \check{\text { ou }} 80$ metres. En reproduction dejuis mai jusqu'en octobre. 
Bugula phumosa (Busk, id., p. iii). - A scoz fringuente daus les draguigers a Astan et dans l'Ouest. En reproduction en juillet et aout.

Bugula avicularia. - Abondanto sous les pierres et les roehers en supplomb sous lespuels elle peul. Au niveau des plus hasses marées, zone des Hymanthatio et des Laminaires.

Les colonies des Bugules sont ammuelles. On n'en troure pas durant l'hiver ou on nen rencontre que des lambeans notts el tombant en morceams. C"est vers la liu de mar's qu'elles reparaisspul ef au mois de juin alles entrent en reproduction el lommissent jusyne vers le milien d'aont, partois jusquar premiers jours de septembre, des larves en abondance.

Apres la reproduction, loutes les colonies neurent el en septembre commencent ì tomber en pieces.

Que deviennent les embryons pendant l'hiver? On les a vus et je les ai rus moi-mène produire des zoécies des le moment de leur fixation; mais il est probable que la colonie naissante reste reduite pendant tout l'hiver à 111 pelit nombre de loges ot ne reprend son déreloppement qu'au printemps. Il arrive soment aussi que les anciens trones degarnis de branches se remettent à bourgeomer apres l'hiver. J'en avais apportí un à Paris au mois d'octobre 1876 . Je l'oubliai sur une étagère dans un flacon d'eau de mer. A la lin de janvier, je le tromai regarni d'une dizaine de loges en pleine activitio végétative.

Buyula flabellata (Busk, id., p. 14). - Se trouve quelpuefois avec la Bugula acicularia dans les grantes maries à Per-Roc'h, à Rollea-SaintPol, mais on la retire le plus sourent arec la drague ou l'engin. Comme lit Buyula avicularia, elle est anmuelle et se detruit après la reproduction qui a lieu atu mois de juillet el d'aoùt.

Flustra chartacea (Johnst., Brit. Zooph., 1847, p. 343). - Très-rare, sur une counille te Pecten prorenant des dinguages d'Astan.

C'arbasea indivisa (Busk, p. i3, pl. LVIII, fig. 3 et 4). - J'hésite à domner à une espèce de Roscoff le nom l'une forme que Busk signale comme n’ayant été trouvée que dans les mers de la Nouvelle-Zélande.

Grpendant il n'est impossihle de trouver ancun caractère différentiel entre mes fohantillons of les dessins de l'autenr anglais. D'autre part, ces mêmes échantillons ne peuvent ètre rapportís it aucune des espèces de Carbasea qui se trourent dans nos parages el spécialement à la Carbasea papyrea.

ilembranipora nembranacea (Busk, p. b6). - Sur les leuilles du Saccorkiza bulbosa, où elles forment de bolles expansious blauches qui atteignent souvent nue très-grande taille. 
Abondante partont dans la zone des Laminaires, particulierment derant l'écurie de Duom, wù on la prend facilement arec la gaffe.

L. moment de la reproduction est le mois de mai.

Mendranipora pritosa (Busk, p.öfi).-- Abondante partout sur le Fucus sematus ot partioulièrement sur le Rhodhymenia palmata, ou chle est facile ì ohserver a cause do la transparence des jeunes fouilies do celie Algue.

Membranipora spinosa (sp. nor.). - Cefte esprice doit ètre cortanement distinguée de la Membranipora pilosa, celle de toutes les Menbranipores dont elle se rapproche le plus. Elle ru difrere par le corps de la loge, qui est plus long, plus grèlr et mieus dégagé des loges enviromnates. De plus, louverture, au lien d'itre simplonient garnic de quatre ou cinf contes épines latérabs et d'une forle médiane inferiente, est protógée par huit longues dents qui, partant des hords, se rencontrent presque an centre et par deux fortes saillies obtuses qui se troureut de part et d'autre de la live operculaire. Le test est crible de ponctuations semblables a celles de la Membranipora pilosa.

Sur le Rhodhymenin palmata dans le chenal.

Hembranipora lineata (Busk, Mar. Polyz., p. 68). - Fréquente sur les fevilles de la Isaninaire sacelnarine dans l'herbier prés l'ile Verte.

Menubranipora Flemingri (Busk, id.). - Commune dums les draguages à Astan sur l'Eschara foliacea.

Lepratia Brongmiarti (Busk, id.).

Lepralia reticuluta (Busk, id.).

Lepralia verncosa (Busk, id.). -- Commune sur les roches, zone des filets.

Lepralia violncea (Busk, id.). - Assez commune sur les pierres à trisbasse ner. Rollea-Saint-Pol, Duslen.

Lepralia coccinea (Busk, $i d$.$) . - Tres-rommune sup les pierres, sur$ l'A scidia sangminolenta et sur les somches des Laminaires, zone des lilets et des Laminaires.

Lepralia linearis (Busk, id.). - Commune sur les roches, depuis la zone des filets jusques dans les eaux profondes.

Lemralia ciliata (Busk, id.). - Commune sur diverses Algues, notamment les Choudrus.

Lepralia variolusa (Busk, id.).

Lepralia nitida (Busk, $i d$.$) . - Tris-commune sur les pierres et sur$ l'A scidia sanguinolenta depuis la zone dos filds jusques dans les eaux profondes.

Lepmalia Peachï(Busk, id.). -- Commune sur les pierres ì hasse mer, Lepratir immominata (Busk, idlo). 
Lipmalin fignlaris Busk, ill.).

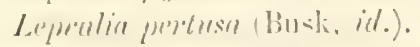

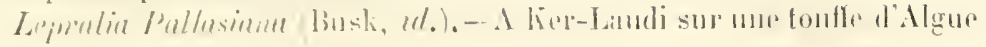

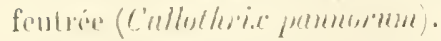

Lepraliu granifure Busk, ill.). - Sur les Moules ans Bisayers.

Lepraliu hynlina Busk, idl.). - Sur diverses Alguros rouges de la zone des Sirraseses ed sur les Chomdrus.

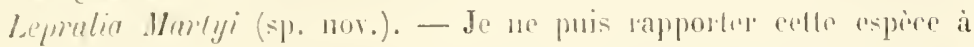

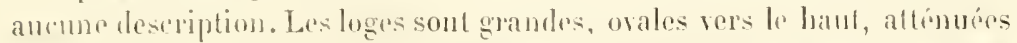

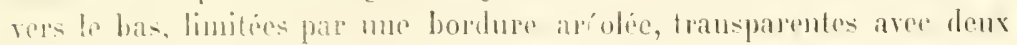

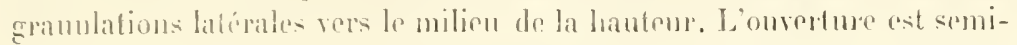

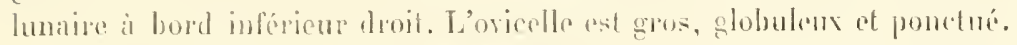

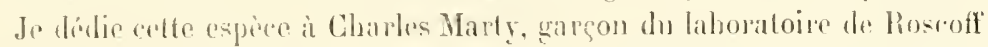

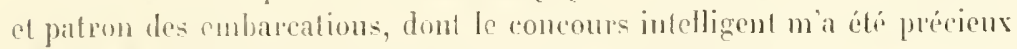
daus mes recherehes it la grove et dans mes draguages.

Lepralia areoluta? (Busk, Mar'. Polyz.).

Cellepora pmicosa (Johnat., Brit. Zooph., 1877, p. 2985). - Extri-

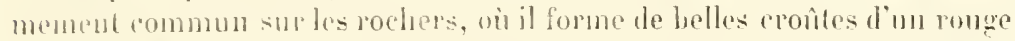
de chair. Hes plus heatux spécinens riennent de Rollea-Saint-bol el de

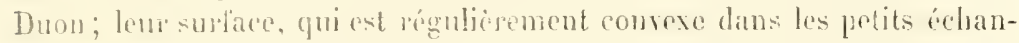
tillons, est alor's ondule at comme forme te plusiems mamelons.

Au nivean des Mimanthatia et phos luss.

(bu procontre daus les eaus profondes me forme yui on differe peu, mais qui, au lieu de śbendre sur les surfacesen expansions larges, forme des nodules entourant des ramuscules d'Algues, de Sargasse, d'I ydraires et de Gorgone.

Celleprora vilrina Busk, Mar. Polyz., p. 87). - Sur le Cladmphora rupescris a la pointe de Bilvidic: (ile de Bas). Commum.

Céllepora ramulosa (Busk, id., p. 87). - Le fimbert ramene cette espece abondinmment de sur les basses de Roeh-Haro dans l'Ouest.

Cellepnra Slienei (Busk, id., 1). 88). - Nème station que le Cellepore ramulosa.

Eschrora foliucea (Busk, id., p. 89). - Tres-commune dans tous les draguages à Astan aussi hien que daus l'ouest et sur lous les fonds roclucus.

J'en ai ohservé deux variétés, l'une rouge, qui est la variété ordinaire et la plus commune, liuntre hlanche, quil ne faut pas confondre arec les parties ancienues et mortes de la précédente. Les potrémitis vivantes en sout de couleur tres-pale ansi bien que les panaches des Polypides épanouis. 
LiEschara foliacea sert de support it de nombreuses esperes de Bryozoaires, notamment au Tubulipora patina, an Jyembranipora Flemingä, ì l'Avenetla fusca co à diverses Lepralia.

Eschara cervicornis (Busk, id., p. 92). - Commune sur les basses de Roe'h-Haro à 30 ou 40 mètres de profondeur parmi les Cellepora Stienei et ramulosa.

Retepora cellulusa (Busk, id., p. 93). - On drague rarement ì Roc'hHaro sans en trourer au milieu des Eschares.

\section{CTÉNOSTONES.}

Sarcochitum polyoum (Johnst., Brit.Zooph., 1817, p.3630).-- Se troure au niveau des hasses mers moyennes sur direrses Algues, notamment lo Rhodlymenia palmata el le Fuens serratus. On le rencontre aussi sur les roches et sur les coquilles. II ne descend qu exceptionneliement juagu'à la zone des Laminaires. Il se prisente sous deur aspects fort différents. Daus sa jeunesse sous forme d'une cronte minee, iransparente, à reflets faiblement irisís s'etalant sur la surface des Algnes. Mais à mesure que la colonie vieillit, son épaisseur derient plus grande. Il lorme alors une masse charnue qui encroùte les fucus au point de les déformer complétement et mème de projeter quelyues lobes en dehors de leu surface. L'aspect est alors terne, gris et spongieux. An mois de mai et juin la surface de la colonie est parsemée de points blanes, quelguefois rosés. Ce sont les aufs qui, lorsqu'ils sont passés à l'blat de larves, s'écliapjent en nombre immense et rivent assez hien dans les cureties. L'éponpe de la reproduction ne se prolonge guere au-dela du mois de juillet.

Cette espece est tris-commmo partout. C'est sur les fucus de la riviere de Pensez qu'elle atteint son plus grand déreloppenent.

C'ycloum papillosum (Johnst., id., P. 36i). - Facile it confondre avec Je Sarcuchitum polyoum, dont il se distingue pourtant à l'wil nu par son aspect rolouté. Sur le Fucus serratus et quelques autres Algues. A PerRoe'll el anx Greyers, zone des Hymanthalia.

Flustrella hispirla (Redfern, Johnston). Syn. Alcyonidium hispidum (Sunit, Bryoz. Mar. Bor. et Artie.). - Très-commune sur he Fucus serratus et sur les souches de C'ystoseira. La reproduction a lieu en juin et juillet. La larve est biralse el hautement organisée. Au nirean des batses mers moyemnes.

Alryonidium hirsutum (Johnst., Brrt. Zooph., 1847, p. 360). - A 


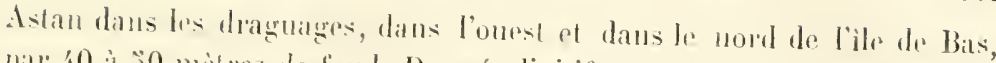

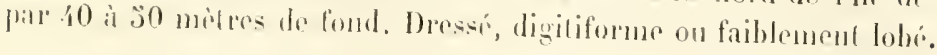

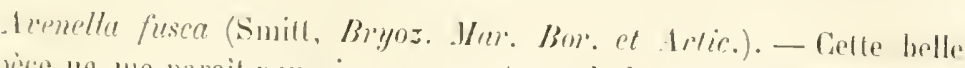

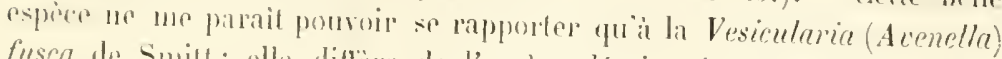

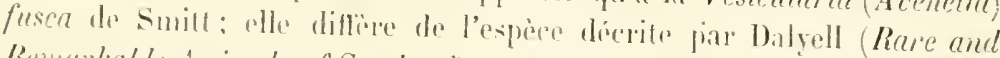

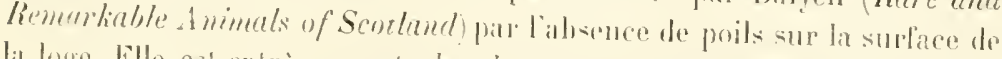

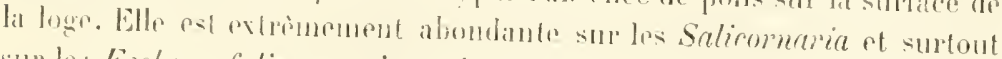
sur les Eschara folincer qui proviemment des draguages; alle forme souront sur la surfice de leurs expansions une sorte de lapis.

Lagenella nutrme nor. sper.). - Cette interessante espèce nest pas rape dims les draguages à Astan on au nord de lile de Bas. On la trouve sur les brimeles de la resiculariu spimosa et surtout sur les feuilles des Nitophyllum qui croissent dins les prolondeurs (Nitophylhum hillix).

Elle se rompose d'un stolon ramprat depourun de cordon central et présentant de distane en distance des unds remplis par un payuet de fibres laumsersales comme dans les Vallieriu.

Sur chaque noend se troure un grompe de zoécies. Les zoécies sont portíes sur un pédoncule ordinairement assez court, mais qui dans me varioté atloint presque la longueur de la zoécir. Ce pédoneule a sa cavité en contimuité aree eclle du stolon et le diaplragme se lrouve à son extrímité au point de jonction avec la zoécie.

La zoécic est de forme prismatique à quatre faces, ses muscles sont disposés comme daus les Valkeria. Cependant deux groupes des muscles pariétur détommés de leurs usages ordinaires s’insérent d'une part sur les parois de la zoécie et de lautre sur le pédoneule, en sorte que par leurs contractions ils impriment à la loge des mouvements de nutation trèsrenarquables, surtout dans les variétés it long pédoneule.

Cette espece un pourrait itro confondue quavec la Laguncula repens de Van Beneden (Layenella regens de Farpe), mais, comme jai jun le constater par l'examen de spécimens reçus de M. Ed. Van Beneden, elle en diffire an ce que la loge de cette dernière espèce n'est point artirulée sur son pédoncule el n'on est pas séparéc par un diaploragme et en ce que l'appareil operculaire de la Laguneule est bilahie, tandis que celui de la Lagenella nutrns est construit comme celui des Valkeria.

J'alkeria cuscuta (Jolnust. Brit. Zooph., 1847, 1). 374). - Cette jolie espere se troure en extrime aboudance sur les mèmes Cladophora rupestris habités par les Cellépores et les Pédicellines à la pointe de Bilvidic.

Je ne lai encore leneontrée que lit soms la forme rampante, at a

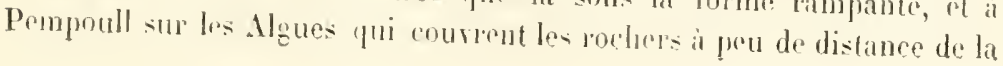


chapelle Sainte-Anne. Je l'ai trouvée sous la forme dressée ou lihre sur un pied de Cystoserio fibrosa, par une lrès-basse mer, à Rollea-SaintPol. Là elle se présentait en belles touffes dont les filaments flotant librement dans l'eau atteignaient justu à 6 et 8 centimibes do longueur. Il n'est cependant pas possible de distinguer spécifiquement eette variété de la première.

La I'alkeria msenta est en reproduction depuis mai juspu'en septembre, mais surtout pendant les mois de juin el juillet.

Bowerbankia imbricata (Johnst., id., 1817. p. 377). - Sous ce nom on doit certainement rúunir la Bowerbankia imbricata de Johnston et la Bourerbankia densa, qui n'en est que la forme jeune.

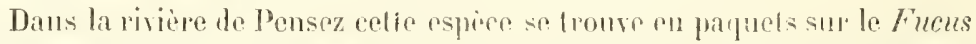
sematus el sur le Fucodium nodosmm. Au printemps sur los touffes rases de cetie derniore espece d'Ague, on tronve abondamment la forme densa do li Bourerbankin composée d'une quantité do loges dressées sur la surface de l'Algue el pressées l'me coutre l'autre de manière à ne former qu'un lapis. Plus tard se délacheul de ce groupe des stolons, puis des rameanx libres qui ponssent des luanehes et, se garnissant de paquets de loges, reproduisent lis forme imbricata.

Entre res dem chats on woit loutes les transitions. Les Polypides de la forme densa ont habitudioment dix luas, cens de la forme imbricata plus sompnt huit, ce qui n'empèche pas que le fail contraire se présente soment. On verra là la ennlirmation de l'opinion de Lovén, qui ne considire le nombre des bras que comme un earactiene de médioere valeur pour la détermination.

J'ai encore tronvé la Bourerlankia imbricata à |Rollea-Saint-Pol sur une lige de Cystoseira fibrosa au milien de la zone des Laminaires. Ce spécimen conservé dans la collection de Roscoff présente ceei de pardiculier, que les zocies, au lien d'ètre disposies sur les liges par parpucts hien accusés, sont distrihuées toul lo long deshranches, de manière qu’il est à prine possible de distiuguer los paquets qui se suivent. Sauf ce délail il est de tout point semblablo aux autres échantillons.

A Per-Roc'h jai trowve la neme cspece rampante sur les Bugules, je l'ai mène rencontrós sur des Vesicularia spinose provenint i'un draguage ¿ Astan, mais e fait est rase et la station préférée de la Bourerbankia imbricata ost le niveau supérieur des hasses uners, la zone des frucus resiculosus ol serratus ol du Fucolium nodosm. C'est dans les fanx liogi-

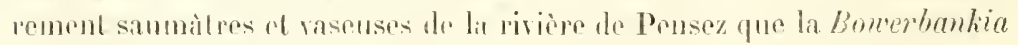

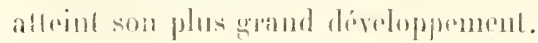

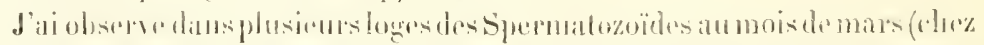

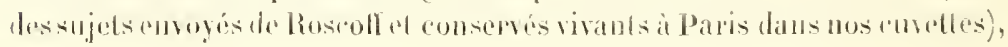


mais je naijamais vu d'exul's à celte épopue. Le veritablo moment de la reproduclion est le mois de juillet arec la fin de juin et le commeneement d'anil. Au mois de juillet, les larves qui normpent tontes les loges doument

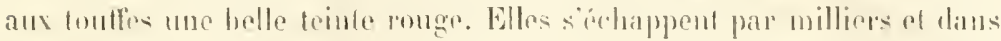

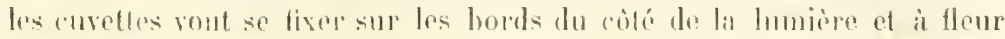
d'eat1. Lit rlles so transforment, et les loges qui on résultent ef qui s'entremelent forment un véritable tapis reproduisant la forme densu.

Vesicularia spinosa (Jolnust., id., 1847, p). 370). - Tres-abendante aux emviroms d'Astan, oil olle ponsar en belles touffes sur les vieilles con-

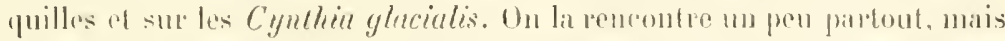

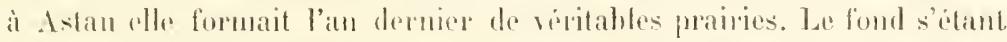

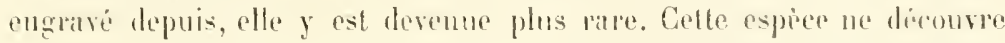
jamais, je ne l'ai pas recueillio à monns de 20 mètes de profondeur. Ello selt do smpport à plusieurs especes, notamment les bicellaria ciliata, Lagenellu repens, I'edicellina echinatu et belgina.

Serialarin lendigera (Johnat., irl., 1847, 1). 369). - Se troure ì tontes les profondeurs, depuis lo nivean do la morte ean sous les muss du laboratoire jusques parmi les oljets dragures it 20 et 30 metres de profondeur. Son grand devoloppement est au niveau des basces mers sur les Algues fibreuses qui se trouvent parmi les zostères. Dans llierloier vaseux de Pempoull elle est extrumement ahondante el forme prespue un gazon en rertains proints.

Serialaria semiconvoluta (Lamk, Heller, Bryoz. des Adrial. Meeres,

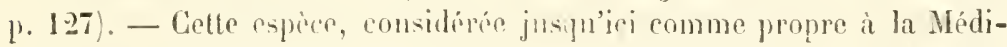
terranée et à l'Adriatique, u'arait pas ancore étí signalée dans la Manelue. Sur une piorre rapportée par un pècheur du nord de l'ile de Bas, jen ai trouvi plucieurs pieds; j'en ai recueilli depuis au trou d'Astan.

\section{ECTOPROCTA.}

Pedicellina echinata Sars, forma typica). - Les tiges sont hérissées de piquants. Fräpuente dans les draguages sur le Vesicularia spinosa, sur les tiges d'Antemularia el sur d'autres hydraires, sur les Bugules aux tris-bassos mers. La zone oceupée par cette variété commence au niveau des plus basses marées el s'iblend jusqu’à 400 ou 60 motres.

l'edicellina eclinata (var. glabua). - Cette varieté ne differe de la Pedicollina echimuta typira que parce que sa tige est habituelloment de pourue de piquants. do me puis cependant la regarder comme une espece distiute. En effet, il mest somrent arriví daus un groupe d’udividus

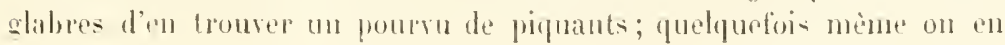


rencontre qui ont des piquants seulement sur une partie de la tongueur ou sur l'un des rîtés de la tige.

Je eonsidipe done relle forme simplement comme la variété littorale de la Pedicellina echizata. On ne le reneontre en effet que dans la zone littorale depuis les Fucus jusqu'ans Hymanthalin. Sa station preférée est à l'ombre dans les flapues d'ean qui ne se vident pas complétement à basse mer, sur la Comallina squammata, et surtont sur les touffes verles de Cladophora rupestris n!ni pendent sous les rochers en surplomb. Sur les filaments de cette Algue on en troure ahomlamment de magnifiepues colonies. A l'extrémité de l'ile de Bas, à la pointe de Bilvidie au milien d'une flaque d'rau neltement circonscrite. A quelques pas eu deçà et au delà on n'en trouve plus tue de rares échantillons.

J'ai trouré celte espiece en reproduction tont lééc, de mai à septembre, mais surtout en mai of juin.

Pedicellina gracilis (Sars). - Cette espere me parait plutot se rapporter à la description do Sars qu'à la Perlicellina belgica de Van Beneden, si toutefois colle-ci est réellement distinete de la premiere.

Hahituellement la tige se composi de demx parties: l'me basilaire, épaisse of pourvue de filures musculaires, l'autre suprérieure, grèle, sans muscles et servant simplement de hampe ou de support à la tète. Quelquefois la hampe se enmpose de deux ou mème de trois articles séparés par un ou deux renflements pourvus de muscles; la tige est alors très-longue.

Cette espèce, heaucoup plus petite que la I'edicellina echinata, est trèsfréquente dans la mème zone ef ordinairement en sa compagnie sur les branches de la Vesicularia spinosn. J'ai observé des larres mûres en septembre, mais les organes génitanx sont risibles en tout temps.

Loxosoma phaseolosomatum (Carl Vogt, Arch. zool. exp., 1. V, p. 30ä). - Ce Lososome, frousé par M. Lemirre, préparatenr au laboratoire de zoologie de la Faculté des seienees, sur l'extrémité infépieure du Phaseolosomu elongatum, a fait l'ohjet d'une momographie dont M. C. Vogt a réuni les ćléments pendant l'été de 1876 au laboratoire de Roscoff' et qu'il a publiée depuis dans les Archives. 


\section{LISTE PAI ORDRE SYSTEMATIOUE}

Tubulipora patina.

$$
\begin{array}{ll}
\text { - } & \text { hispicla. } \\
\text { - } & \text { serpens. } \\
\text { - } & \text { flabellaris. }
\end{array}
$$

Discoporella....

Alecto dilatans.

- granulata.

- major.

Diastopora obelia.

Pusfulipora deflexa.

Crisia denticulata.

- eburnea.

- aculeata.

Crisidia cornuta.

Serupocellaria scruposa.

Canda reptans.

Salicomaria farciminoides.

Picellaria eiliata.

Bugula plumosa.

$$
\text { - avicularia. }
$$

- flabellata.

Flustra cbartacea.

Carbasea indivisa.

Membranipora membranacea.

$$
\begin{array}{ll}
\text { - } & \text { pilosa. } \\
\text { - } & \text { spimosa. } \\
\text { - } & \text { lineita. } \\
\text { Flemingii. }
\end{array}
$$

Caberea Boryi.

- Hookeri.

Eschara foliacea

$$
\text { - cervicoruis. }
$$

Retepora cellulosa.

Hippothoa divaricata.

Lepralia Brongniarti.

$$
\text { - reticulata. }
$$

Lepralia riolacea.

- coccineil.

- linearis.

- ciliata.

- variolosa.

- nitida.

- Peachii.

- inuominata.

- figularis.

- pertusa

- Pallasiana.

- granifera.

- hyalina.

- Martyi.

- ireolata?

Cellepora pumicosa.

- vitriua.

- ramulosa.

- Skenei.

Eucratea chelata.

Anguinaria spatulata.

Beania mirabilis.

Sareochitum polyoum.

Cycloum papillosum.

Flustrella hispida.

Aleyonidium lıirsutum.

Avenella fusca.

Lagenella uutans.

Valkeria cuscuta.

Bowerbantia imbricata.

Vesieularia spinosa.

Serialaria lendigera.

Serialaria semiconvoluta.

Pedicellina eehinatatypica.

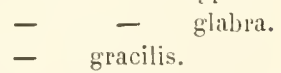

Loxosoma phascolosomatum. 


\section{DISTRIBUTION EN PIOFONDEUR}

\author{
ESPE்CE \\ DE LA ZONE LITTORALE \\ dicuuvrant \\ aux plus basses marées.
}

Alecto dilataus.

Tubulipora serpens.

Canda reptans.

Eucratea chelata.

Anguinariil spatulata.

Bugula avicularia.

Membranipora nembra nacea.

.lembranipora pilosa.

spinosi.

Lepralia verucosa.

- granifera.

- hyalina.

-- Marlyi.

Cellepora vitrina.

Flustrella hispida.

Sarcochitum polyoum.

Cycloum japiliosum.

Bowerbanlia imbricala.

Valkeria cuseuta.

Pedicellina echiuata, var. glabra.

Loxosoma phascolosomatum.

\author{
ESPECES \\ RÉPANDLES DANS TES DELS \\ ZONES.
}

Crisia denticulata.

- elunrua.

- acule.ta.

Crisidia cormuta.

Serupocellaria seruposa.

Hippothoa divaricata.

Beania mirabilis.

Bugula flabeflata.

Caberea Ilookeri.

Membranipora lineata.

Lepralia nitida.

Cellepora pumicosa.

Serialaria lendigera.
ESPECES

DE LA ZONE PROFOXDE ne décusrant jamais.

T'Lblipora palina.

$$
\text { - hispida. }
$$

Alecto major.

- grautusala.

Salicornaria fareiminoides.

Ciaberea Boryi.

Bicellaria ciliala.

bugula plumosa.

Membranipora Fleningii.

Carbasea indivisa.

Fusha charlacea.

Eschara folincea.

Retejora cellulosa.

Eschara cervicornis.

Cellepora ramulosa.

Cellepora Skinei.

Aleyonidium hirsutum.

Avenella lusea.

Lagenella nutans.

Vesieularia spinosa.

Serialaria semiconvolula.

L'edicellinis echinata typica.

l'edicellina gracilis. 


\section{EXPLICATION UES PLANCIIES}

\section{PLANCIIE V'I.}

Fig. 1. Reproduction de la figure 3, pl. Xill du mémoire de lirilz Müller.

Fug. 2. Tejroduclion de la figure 7, pl. Xll d du mémoire de Firitz Müller.

Fug. 3. ()e. 4, Haptnak; obj. 4, Hartuah. Figure destince à montrer les rehtions du funicule aree un très-jenne bourgeon; $b$, bourgeou; funienle surchargí do granulations dans sal partic moyenue; cbr, corps brun religué sur les jarois de l'endocyste; $c p$, cordon principal chargé de granulations surtont dans sal partie iulérieure; $a$, displuragme; $p$, perforation.

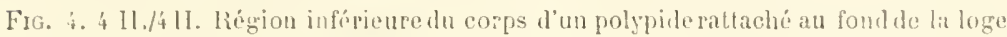
parle funicule; gs, gésier'; rectum; $e$, estomac; funicule s'atfachant vers l'extrémite du carem de lestomac et se prolongeant nu peu sur la laee ventrale de cet organe.

Fig. :. 2 1I./911. Portion du funicule arraché du fond de la loge et eneore adlúrent à lestomac, traité par l'aeide chlorlydrique faible, pour montrer les cellules fusiformes qui le constituent.

Fıg. 6. 2 [1./3 11. Deux coupes ont été faites en travers de la tige an-dessus el atudessons d'un noud, de manière ì i oler un diaphragme qui est vu de face, p, prerforation cenlrale; $i$, impression laissée par le ganglion; $c p$, debris du corlon principal; ec, lambeau de la paroi latérale de la loge rahatu par fo poids de la lame?le couri'tobjet.

Fig. †. Rapuort des dimensions du coplon nerveux el de la perforation du diapluragne.

Fig. S. 2 II./9 H. Deux cellules fusiformes du cordon central isofies, pour montrer qu’il ne part aucune fibre de leurs extrénités et tratées par l'acide osmique; gr, amas de granulations et granulations rélritigentes isolées.

\section{PLA.TCHE VII.}

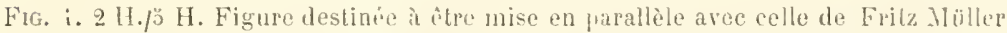
pour montrer que les prétendus ganglions ne sont pas, comme lat figuré cé anteur, des renflements contimus des corduns nerveux, mais qu’ils sont divisés en deux moities par des diaphragmes perers senlement en lenr centre d'une fine jerforation. $g$, ganglions; $p$, perforation d'un diapluragme; $c p$, cordon principal el ses ramifications.

Fıg. 2. \& H./' H. Portion de plexus prise au milieu d'une lige et montrant qu'en eerlains points ses branches vont s'attachep largement ì l'andoeyste.

Fus. 3. \& H./ H. Aprarenee de ganglion présentée par la juxtaposition des deux amas de matière granulense résultant de l'épatement du cordon ceutral sur les deux faces du diaphrigme.

Fig. '́. \& H.J' Il. Perforation d'un diaphragme vue de trois quarts.

FIG. 3. 2 II.99 H. Trouson du cordon centril de la tige, traité par l'acide chlorhydrique faible pour montrer teo celtules finsilormes dont il se compose. $t c$, ectocyste de la loge; en, endocyste détaché de l'aetocyste par l'action de l'acide. Le còté

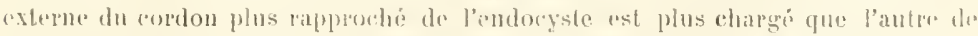

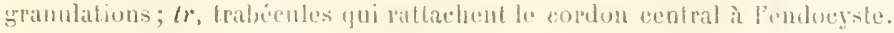

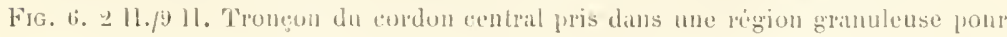
montrer les mudifications que ses eellules peuvent subir. Du còté gauche, on voit 
quelques ecllules fusiformes encore reconnaissables, mais du cóté externe on ne soapconne lenr forme que par la direction des séries de granules refringents qui les rempli-senl.

\section{PLANCHE VIII.}

Les flgures 1, 2, 3 et i représentent deux loges de Bugula flabellata, dessinćes i la chambre claire les 16, 23, 26 janviep et 1er février 1877.

Fig. 1. 2 II./i II. 16 janvier. Le polypicle E est en train de s’épanouir, il est encore de couleur dâle; le polypile F est plus foncé, des granules bruns tournent dans le rectum; $b$, jeune bourgeon; $a$, ariculaire en voie de formation.

Fig. 2. 2 11./4 H. 23 janvier. E est eneore visant, mais il a bruni; F est presque réduit a l'état de corps brun; la loge ot le bourgeon $b$ n'ont pas été représentés dans eette figure, faute le pla"e.

I1G. 3. 2 II./4 11. 26 janvier. E s'est préduit en une masse ovoïle amorphe jaunàme; F a dimiuué de volume.

Fis. 4. 2 H./F H. fer février, E et F, sont devenus de véritables eorps bruns.

Les figures 5, 6 ct 7 représentent une méme loge do Bowerbankia imbricata, des. sinée ì la chambre claire les 5,6 et 10 juillet 1877 .

Frr. :3. 2 II./4 Il. 3 juillet. Le polypide, qui était vivant la surveille, est déjà llétri et la collerefte est presque complitement sortie au dehors.

Jíg. b. 2 H./i II. 6 juilet. La collepette est complétement déragince, les restes du polypide se réduisent et s'affaissent.

Fif. 7. 2 II./i Il. 10 juillet. Le corps hrun est paracheré et entouré de quelques granules plus elairs, $g r$.

FIG. 8. 211.j3 II. Pieprésente le exeım d'un mème polypide de Bicellaria ciliata à quelques minutes d'intervalle. En A, le eanal $c$ est très-étroit; en B, pell de temps après il est agrandi. Ces danx figures doivent être comparées aux figures 9 et 10 , qui reproduisent les dessins de Hincks; elles montrent neltemenl que la eonstrietion qui produit le canal c n’intéresse pas le contour extérieur des parois stomacales.

Fıg. 9 ei 10. Extrémité d'un cxcum de Bicellaria ciliata, d'après Hincks (Q. J. micr. sc., t. XIll, p]. II, fig. 6 et 7).

Fia. 11. 2 II./9 H. Epithélium de la région inférienre du excum de la Bugula flabellata. Il est formé de eellules polygonales eontenant chacune un ou deux granules bruns.

FIG. 12. 2 I1.17 II. Corps bruns tiré d'me loge d'Eucratea chelata et environné des : granules jauntres que Repiacliofl regarde comme les restes des tentaeules du bourgeon $g r$.

Fig. 13. 2H./7 H. Corps brum tiró d'une loge de Bowerbankia imbricala et montrant au milieu de sa substance plusieurs corps étraugers et un certain nombre des plaques dures qui revêtent le gésier du polypide.

\section{PLANCHE IX.}

Les figures 1, z et 3 représentent une mème loge d'Eucratea chelata, dessinée à la chambre claire lo ler, le 2 pl le 3 juin 1877.

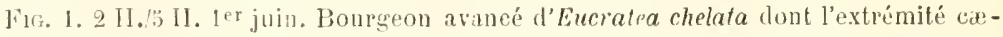
cale est en rapport avec un corps brun. $c b$, eorps brun; $g r$, granules qui l'environnent; $f$, lunieule; $m$, méconium; $\mathrm{cl}$, cloison; $\mathrm{cp}$, cordon principal de l'endosarque. 
FIc. 2. 2 II./: II. 2 juin. Le cotps brun traversant la paroi intestinale usl passé ditn. la cavité du coecum, quelgues gramules jannes gr sont restés sur la paroi au point oì s'est effectué le passage; plusieurs autres sont dújit dans le lectum.

Fut. 3. 2 H//3 Il. 3 juin. Le corps brun est passé dans le rectum, où se troutent encore le méconium el plusieurs gramules. 11 est encore entouré de sil menbrane. Le polypide s'étant accidentellement flétri, l'évacuation du corps brun n'a pu atoir lieu.

Fıg. 4. 211/3̈ll. Polypile qui élait le 7 juin absolument dan les memes conditions que le précédent; son rectum était, ì cette date, distondu par un eorps brun; les, il élait dans l'état que représente lit figure 4 , son rectum ne contenant plus que quelques gramules.

FlG. 3.. 2 H./' Il. Bonrgeon de Lepralia granifera se développant sur un corps brun.

Fig. 6. 2 II./4 H. Le bourgeon enlonre le conjs brun de sa substance.

Fı, 7. $211 . / 4$ Ii. Le corps brun est maintenant dans la cavité stomacale du polypide; $m$, méconium.

Fig. 8. 2 H./h II. Le corps bruns est résolu en granules qui sont éracués peu ì peu; un paquet de ces granules occupe le rectum.

Les figures 9 et 10 représentent la mime loge de lialleria cuscula, dessinée à la chambre claire le 2 et le 6 mars 18i7, dans des positions un pen différentes, ce qui fait que les corps bruns ne se présentent pas de mème.

FIG. 9. 2 [1/5̈IL. Jeune bourgeon; $m$, méconium; cst, cavité stomacale; f, funicule; $g l$, gaine tentaculaire.

Fig. 10. 211./3 II. Le polypide est presque adulte; le corps brun est resté ce qu'il était, n’a pios passé tlans la cavité stomacale et n’a pas pris part an développement.

Fır. 11. 2 H./9 11. Ciellules détachées de l'épithélium stomacal de la Bowerbantia imiricata. Elles sont cliargées de granules bruns.

Fı. 12. 2 H./9 H. Cellules délachées de l'épithélium stomacal de la Bugrila flabellata.

\section{PLANCHE X.}

Fig. 1. 2 H./7 H. Deux loges de Bicellaria ciliata en reproduction; ov, ov, œufs appendus au ceecum stomacal et dépendant du funicule; $\mathrm{cm}, \mathrm{cm}$, cellules mères les zoospermes développées dans la parlie basse du funicule; celles de la loge supérieure sont déjà mùres et laissent passer la quene des zoospermes.

EIG. 2. 2 H./9 H. Loge de Falkeria cuscula en reproduction. Le polypide n'est pas encore adulte, car son rectum contienl encore un méconium; $t$, testicule formé par l'accumulalion des cellules mères dèveloppées dans le sein du funicule; $o v$, ovaire contenant tleux aufs et altaché au sommet du funicule au contact du eæcum stomacal.

FIG. 3. 2 H 17 H. Loge de Bowerbankia imbricala au moment de la reproduction. L.e polypide est adulte. ov, ovaire contenant deux oufs et développé dans cette partie du funicule, qui est adhérente au cœeum slomacal; cm, cellules mères des zoospermes en petit nombre, encore adhérentes an funicule.

FıG. 4. 2 I1.19 II. Jeune loge de Valkeria cuscula, dans laquelle les éléments générateurs se déreloppent eu même temps que le polypide et dans le sein du tissu funiculaire. Les oul's ne sunt pas encole distinets ; $\mathrm{cm}$, cellules mères; mpv, nuuscles pariéto-vaginaux; $g t$, gaine tentaculaire; $c$, masse dans laquelle naitra la collerette.

FIr. 3. 2 [1./711. Jeme bourgeon de Bugula aricularia sous lequel on distingue dans la dépendance du funicule : ov, un ovaire; 00, deux cufs déjà formés.

Fig. 6. 2 H.,7 II. 1)eux œufs appendus au funicule dans la bugula flatellata. 
Fir. 7. 2A. 7 II. Granules pésultand de la Iranformalion dés fiéments de l'endosarque et prèts à se dètacher pour former des cellutes fottantes, dans la Lagenella mulans.

Frg. S. 2 H., 9 H. Cellules flothates de la Bowerbankia imbricala.

Fig. 9. $211 / 711$, Mlèmes granuies dans la Bugula avicularia.

F1G. 10. 211.9 H. Cellules flottantes de la Bicellaria ciliata.

\section{PLANCHE XI}

F1G. 1. 2 II./3 II. Loge de Lepralia Marlyi avec son ovicelle. La loge renferme un jeune polypide en voie de développement el contenant dans sou estomac un corps blun. A còlé se trouve un ovaire dans lequel se volent deux onfs: l'un presque mûr, d'un rouge vif; l'autre beaucoup plus petit et encore incolore. Tous deux sont pourvus de la vésicule germinative. Dans l'ovicelle se trouve une larve ciliée prète ì s'échapper.

FJG. 2. 2 11./7 11. Représente une loge de Bicellaria ciliala, dans laquelle se Lronvent simultúment un ovaire funiculaire ov, $f$ appendu an cœenm du polypide et un ovaire parićtal ov, $p$, accolé à la paroi interue de la loge. Dans le sein du funieule et au-dessous de l'ovaire funiculaire on remarque des cellules mères de zoospermes, $\mathrm{cm}$.

FIg. 3. 2 II.17 H. Très-jeune ovaire de Leprulia Marlyi, accolé à la paroi de la loge dons la région inférieure. De sa surface se détaclue le funicule fn qui se rend au corps brull.

Fig. 4. ב II./. II Jeune loge d'Eucratea chelaia dans laquelle se déreloppe, exelusiremenl aux dépens de la substance du funicnle $f n$, un jeune polypide $p$; en, endocyste celluleux; $f n, f n$, enclosarque traversant la cloison entre les loges; gi, gaine tentaculaire en voie de formation.

FıG. 5. 211.7 H. Loge de Valkeria cuscula déjà pourvue de trois corps bruns, et dans laquelle bourgeonue un nouveau polypide daus le sein du funicule et ì distance appréciable de l'endocyste.

Fug. 6. $211 / 7$ H. Formation des cufs dans la Lepralia Martyi. ov, premier ovaire; ov, second oraire ou celiule mère d'œuls. Le premier est creusé d'une cavité dans lapuelle se roient deux cufs. Le second est encore rudimentaire.

Frg. 7. 2 H./9 11. Ovaire pariétal de ia licellaria cilinta, vu sous un plus fort grossissement.

F1G. S. 2 11.17 H. Ovaire funiculaire de la Bicellaria ciliatc.

F1G. 9. 211.19 H. Cordon principal de Buwerlankia imbricata monlrant les cellules boursouflies dont l'une est prête ì s' détacher pour devenir une cellule flottinte.

Fu. 10. 2 II./9 H. Zoosperme de Lepralia Marlyi en train de se débarrasser de sa cellule mère.

Fig. 11. 2 H.17 H. Première ébaucha d'un bourgeon de vésiculaire sur la paroi d'uno loge ancienne au contach du funicule. On voit un paquet de cellules résultant de la prolifération dés éléments du funicule.

Fig. 12. 2 11./7 11. Les cellules se répartissent en deux conches et s'éfalent pour former une sorte de couronne de gros éléments disposés autour d'un tissu central plus fin.

Fit. 13. 2 II./7 H. Elat plus avancé du même bonrgeon. Au centre se voil la fente qui indique la cavité digestive.

FIG. 14. 211.17 H. Zoosperme de Bowerbankia imbricala formé d'une grosse têto en massue et d'une longue quene fliforme et flexuense. 


\section{Pl.d.iCHE: XI1.}

lig 1. 2 11./9 11. Extrémité végélative d'un stolon de pédicelline montrant les eellules eylindriques de l'endocyste se raccoureissant graduellement it mesure quon s'áloigne du póle do la calotfe, et atu entres, les cellules détachées et encore arrondies dans ceite région, qui constituent le parenchyme.

FIci. 2. 2 II.9 II. Extrémité végítative d’un stolon de Rowerbankia inbricata mise en parallèle avec celle de la pédicelline pour montrer la similitude du développenturut.

Fig. 3. 2 11./7 II. . Lème extrémité sur une plus grande longueur et sous mu plus faible grossissement, four montrer comment le cordou principal se forme aux dipens des cellules de l'endocyste terminal.

F1G. 4. 2 II.j9 II. Extrémité d'une tige de Pedicellina gracilis, pour montrer audessous de lil eouche eclluleuse de l'endocyste les cellules fusiformes du parenchyme.

Fig. 5. 2 II./7 H. Extrémité végélative d'un stolon d'Eurratea chelata, pour montrer que l'endosarque accolé au bord inlerne de la loge se forme anssi aux dépens des cellules de l'endoeyste terminal.

FıG. 6.2 H./9 H. Portion moyenne d'un stolon de Pedicellina echinala, pour montrer les cellules fusiformes anastomosées dont est eonstitué son parenchyme.

Fig. 7. 2 II./9 JI. Endoeyste de l'extrémité d'une lige de Pedicellina gracilis.

Fig. S. 2 II /9 fI. Struclure de l'endosarque de la Bugula avicularia.

FIs. 9. 2 H.17 H. Jeune tige de Pelicellina echinala au sommet de laquelle, arant l'apparition du diaplsragme, un polypide, eneore réduit à six cellules, bonrgeonne aux lépens de l'endoȩ̣ste.

FIG. 10. 2 II./7 II. Bourgeon plus avancé; les premiers linéaments du diaphragme commencent ì se dessiner.

Fıg. 11. 2 H./9 11. Première ébanche d’une zoécie dans la resicularia spinosa. Ce u'est encore qu'nne ampoule soulevant la surface de l'eetocyste et doublée intéricurement d'une eonche d'endocyste celluleux, ì l'intérieur on renargue une trałnee de granules appartenant à l'endosarque.

FIG. 12. 2 H./7 II. Les granules internes devienuent plus nombreux.

Fig. 13. $2 \mathrm{H} / 7 \mathrm{H}$. Ils se groupent au milieu de l'ampoule.

Fig. 14. 2 H./7 H. Ils se rémissent en un amas central qui est nettement en conlinuité arec l'endosarque dı stolon.

Fig. 15.2 H./7 II. Les cellules se sont multipliées et sont devenues plus fines, le bourgeon ressemble à ee moment ì une masse de sarcorle homogène. Le diaphragme est apparent.

Fig. 16. 2 H./7 H. Mleme stade après trailement par l'alcool, le bourgeon est nettement séparé de l'endocyste et relié au funicule, dont il est comme une dilatation.

FIG. 17. 2 H./7 H. Apparilion de la fente digestive.

\section{PLANCHE XIII.}

FiG. 1. 2 H./7 H. Loge d'Eucratea chelala montrant nettement un jeune bourgeon qui se développe dans le sein de l'endosarque au-dessus du corps brun.

Fig. 2. 2 H./5 H. Polypide d'Eucratea développé sur le funicule indépendamment du corps brun et de l'endocyste.

FIG. 3. 4 11./2 II. Larve de Flustrella hispida représentée de profil ; p, ébauche du polypide; $m r$, muscles rétracteurs; $c s$, bouton pourvu de soies. 
Fig. 4. 4 II. 2 1I. La larve s'est désorganisée et est représentée le face à cheval sur ses deux valves et réduite à une masse de protoplasma gramuleux dans lequel apparait un bourgeon de polypide.

Fıg. ว.. 211./5 H. Loge de Valkeria cuscula renfermant deux œufs o encore attachés aux restes cb du polypide qui les a produits. Sur la paroi, au contact du funicule, se développe un bourgeon de polypide. Les œufs présentent leur résieule germinative. La loge est complétement close et ne renferme plus de spermatozoïdes.

FIG. 6. 2 II/3 II. Loge de Vallieria cuscuta observíe le 18 juillet. Elle ne contient qu'un cut arrivé ì son développement complet el montrant encore sa vésicule germinative; dans le haut de la tige, se voit un petit polypide qui a développé un nouvel appareil operculaire et ouvert la loge.

Fig. 7. 2 H./3 H. Même loge le 19 juillet. L'œuf qui était hier au-dessons du po-

lypide auxiliaire est maintenant au-dessus. Il a pris place dans sa gainc tentaculaire et perdu sa tache germinative; $m$, méconium. L'œuf est maintenant en communication avee l'intérieur.

Fig. 8. 2 H./5 H. Le polypide auxiliaire est atrophié, mais on reconnaît encore son méconium $m$, et ses muscles rétracteurs subsistent an-lessus de lui, l'œuf fécondé s'est segmeuté en huit splières.

FIG. 9. 2 H./3̈ II. La larve a paursuivi son développement et est maintenant presque adulte; elle est tonjours renfermée dans la gaine tentaculaire et mue par les muscles rétracleur's mgr et le funicule, elle monte et descend dans la loge, se portant souvent jusqu'à l'entrée. Áu-dessous d'elle, on aperçoit cncore le méconium $m$ du polypide auxiliaire.

F'IG. 10. 2 II. 17 11. Lirve ćclose de Valkeria cuscula. An eentre, me masse grise plus fortement ciliée que le reste an niveau de l'échancrure. Autour, zone de cellules rayonnantes en éventail; plus en dehors, zone de protoplasme granuleux limitée par un épithélium ciliaire général. 
$4=45$

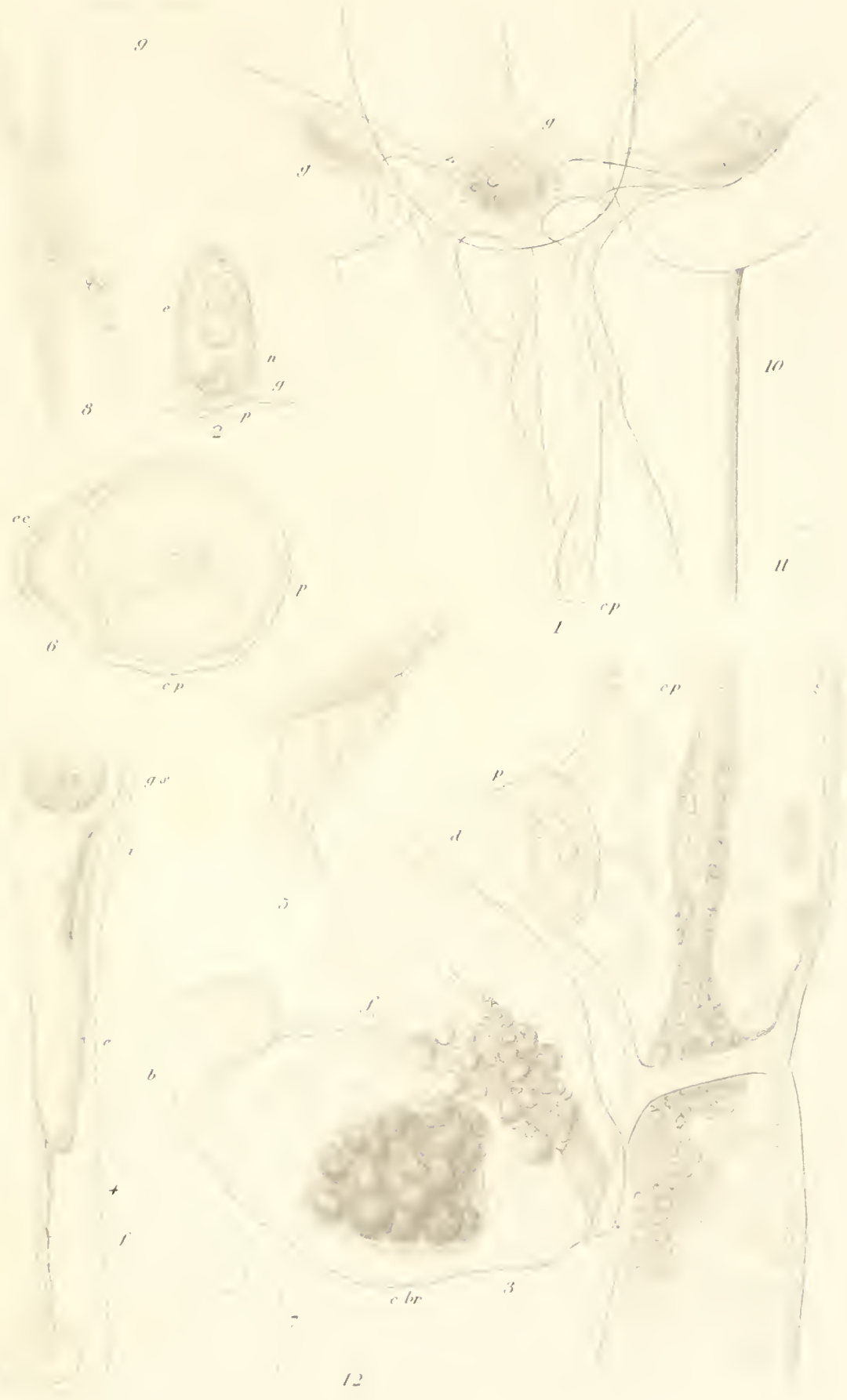





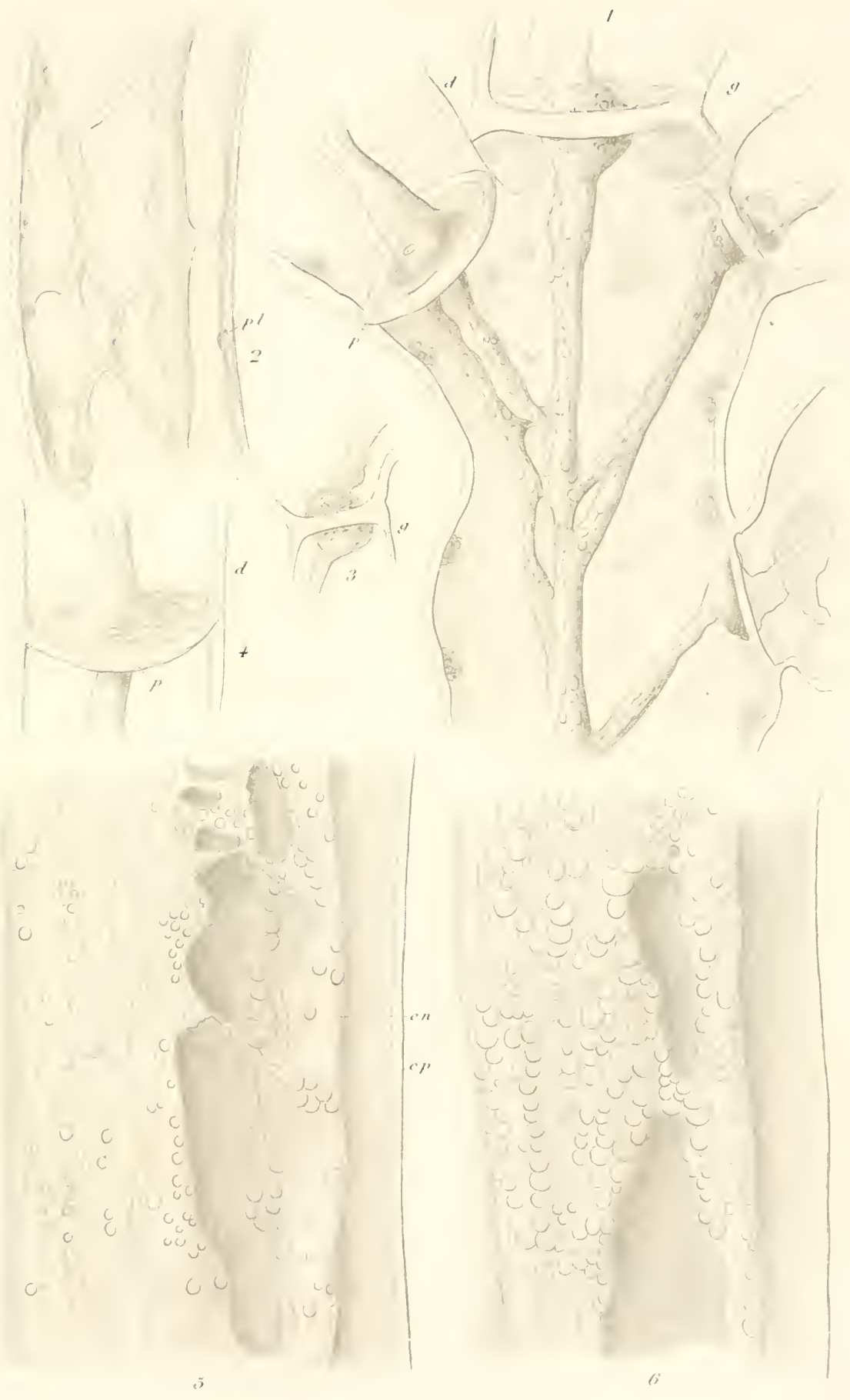





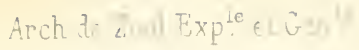

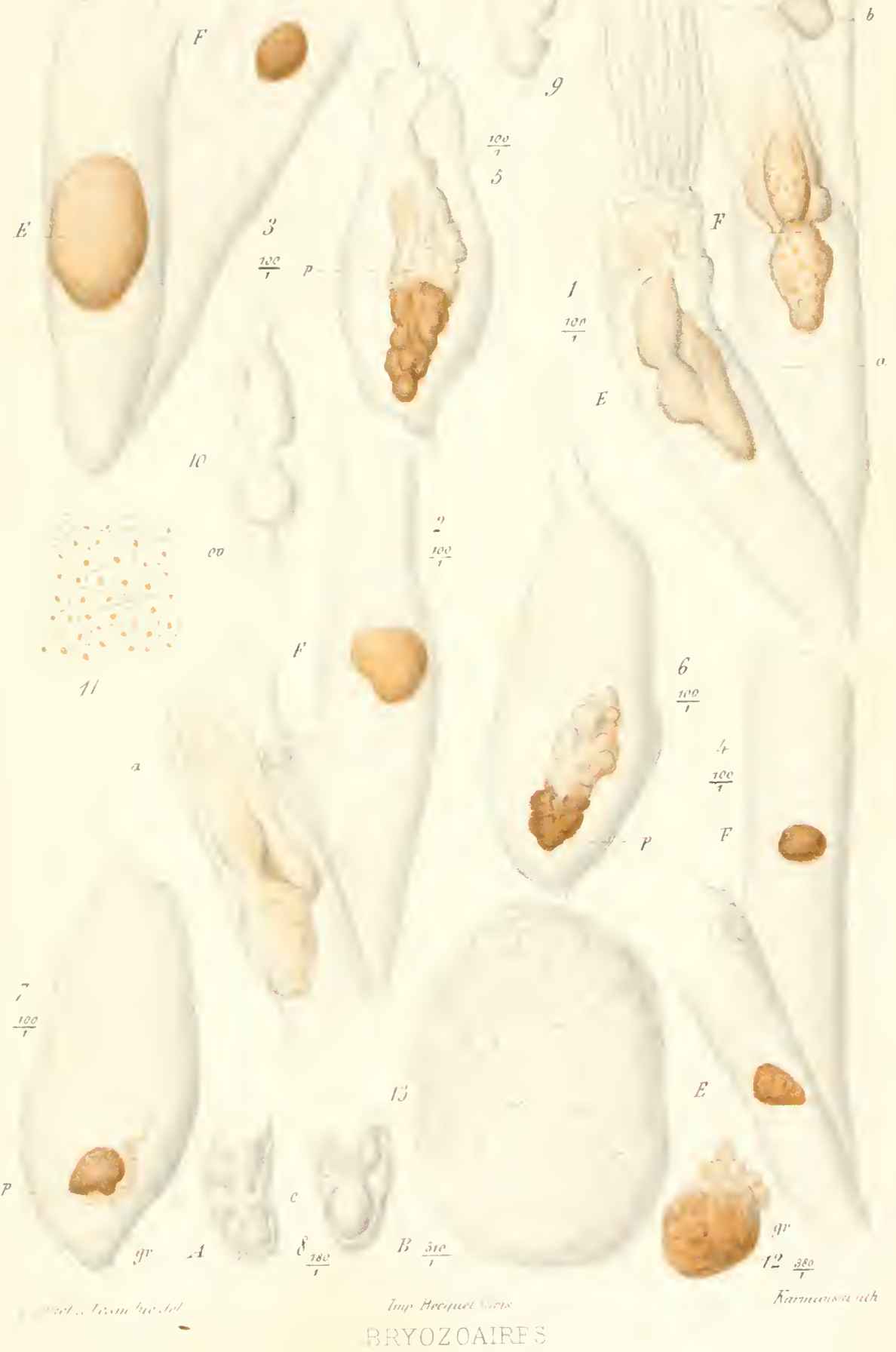



In h de oo Fxple et Gen ${ }^{\text {ie }}$

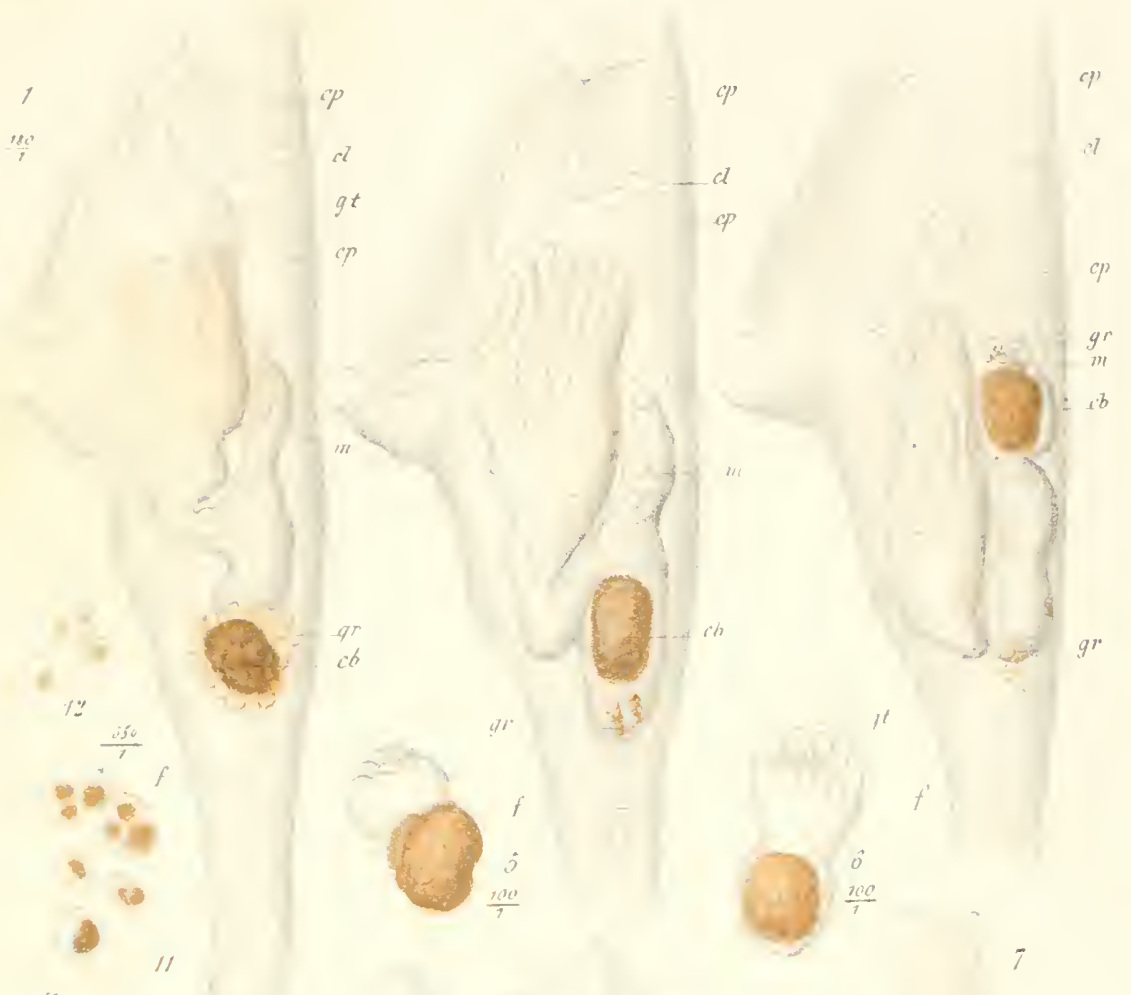

$\frac{550}{1}$

n.

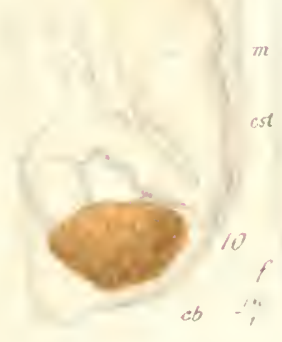

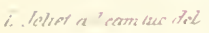
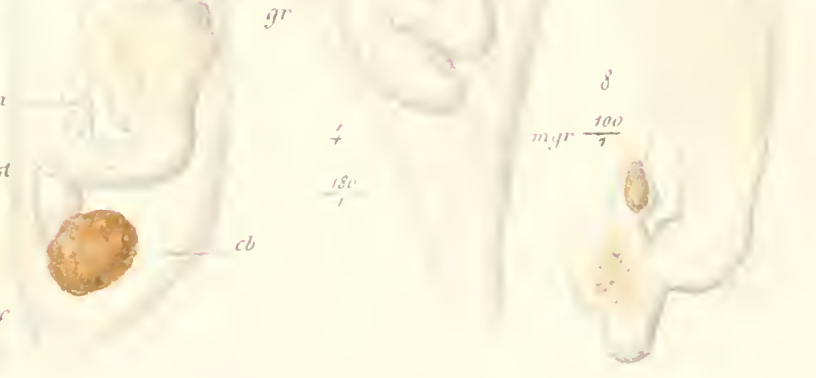

limp. Becauel fizys

Karmermatic lish

BRYOZOAIRES

Ròie dit comps :" 



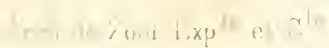

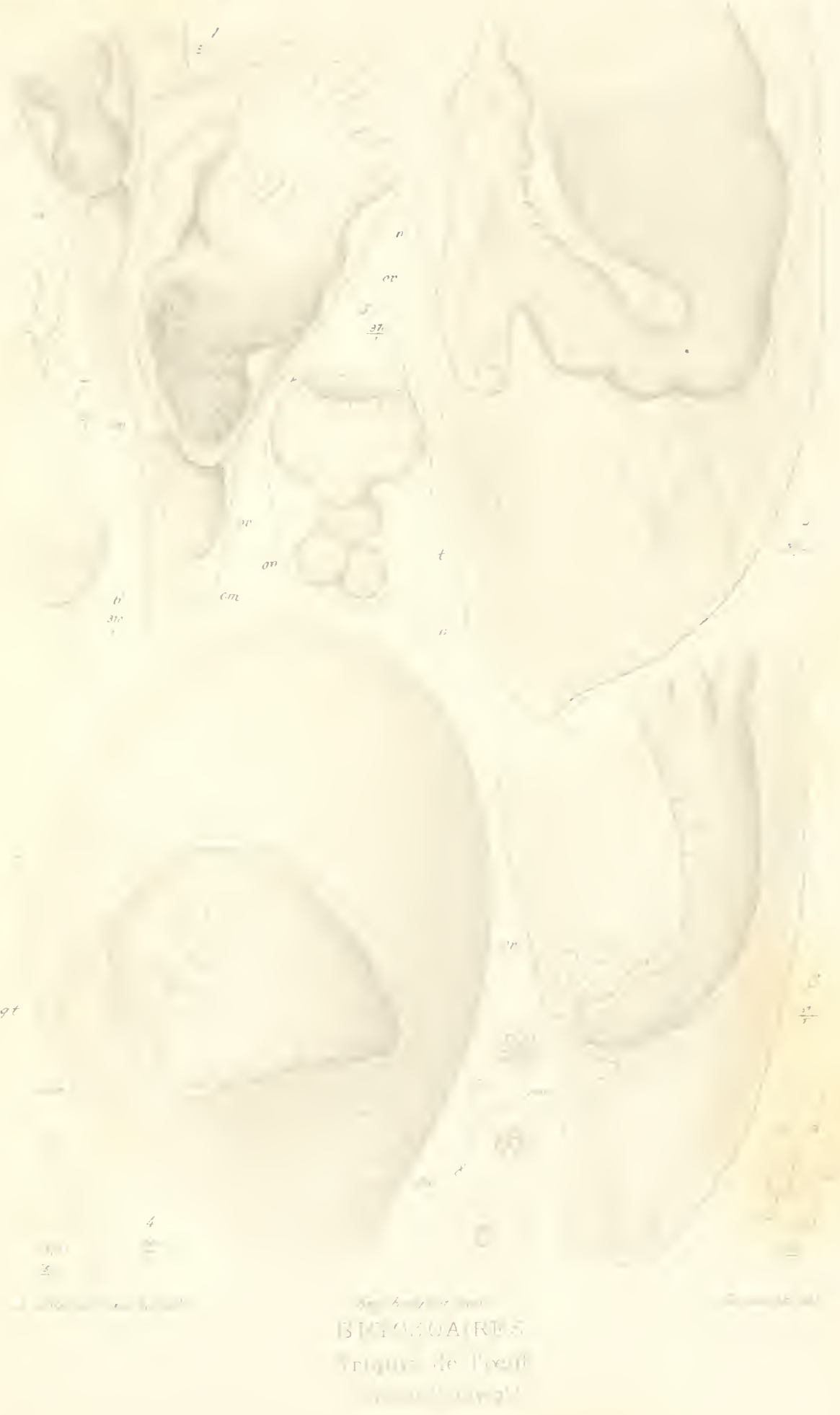





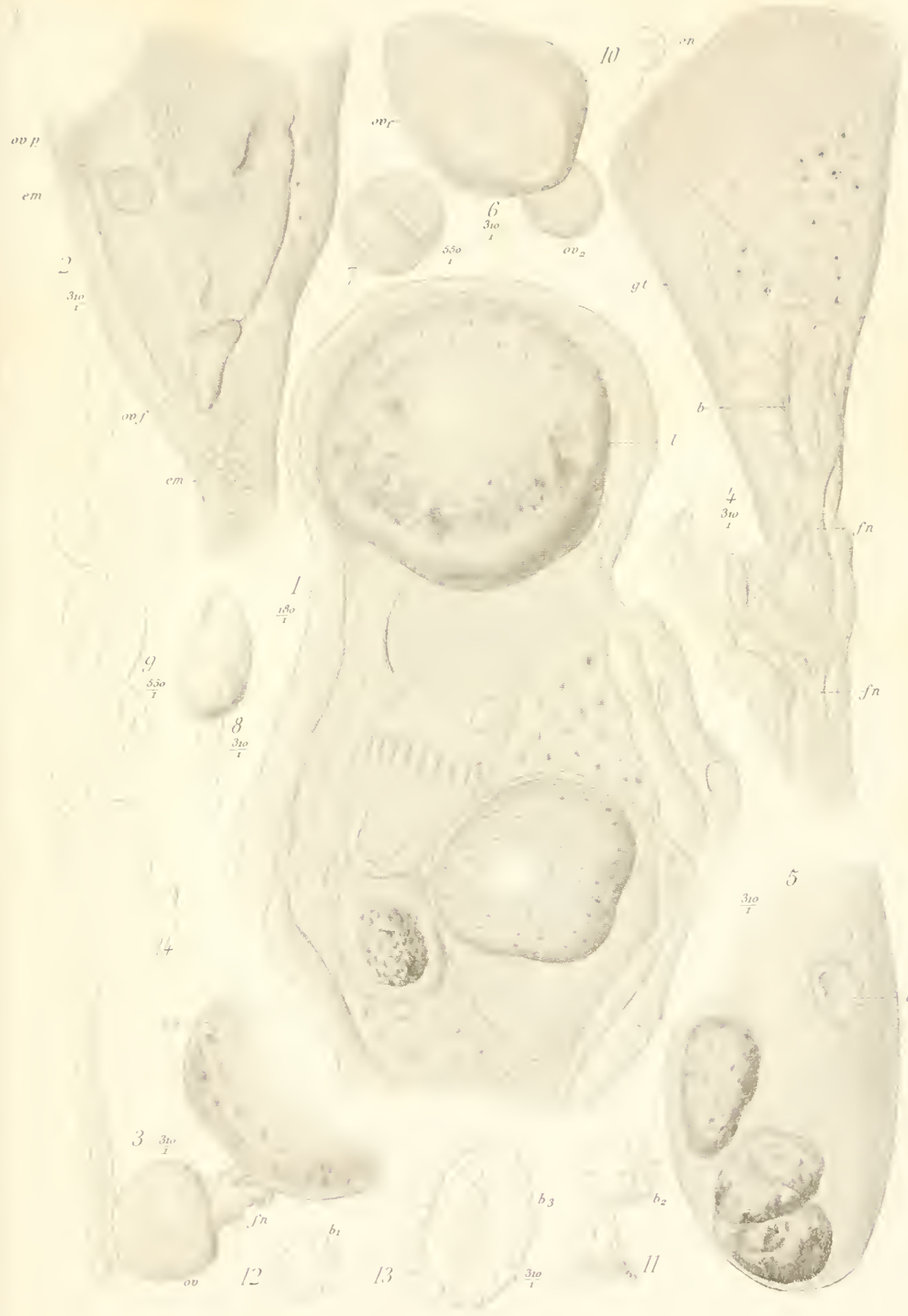





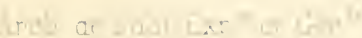

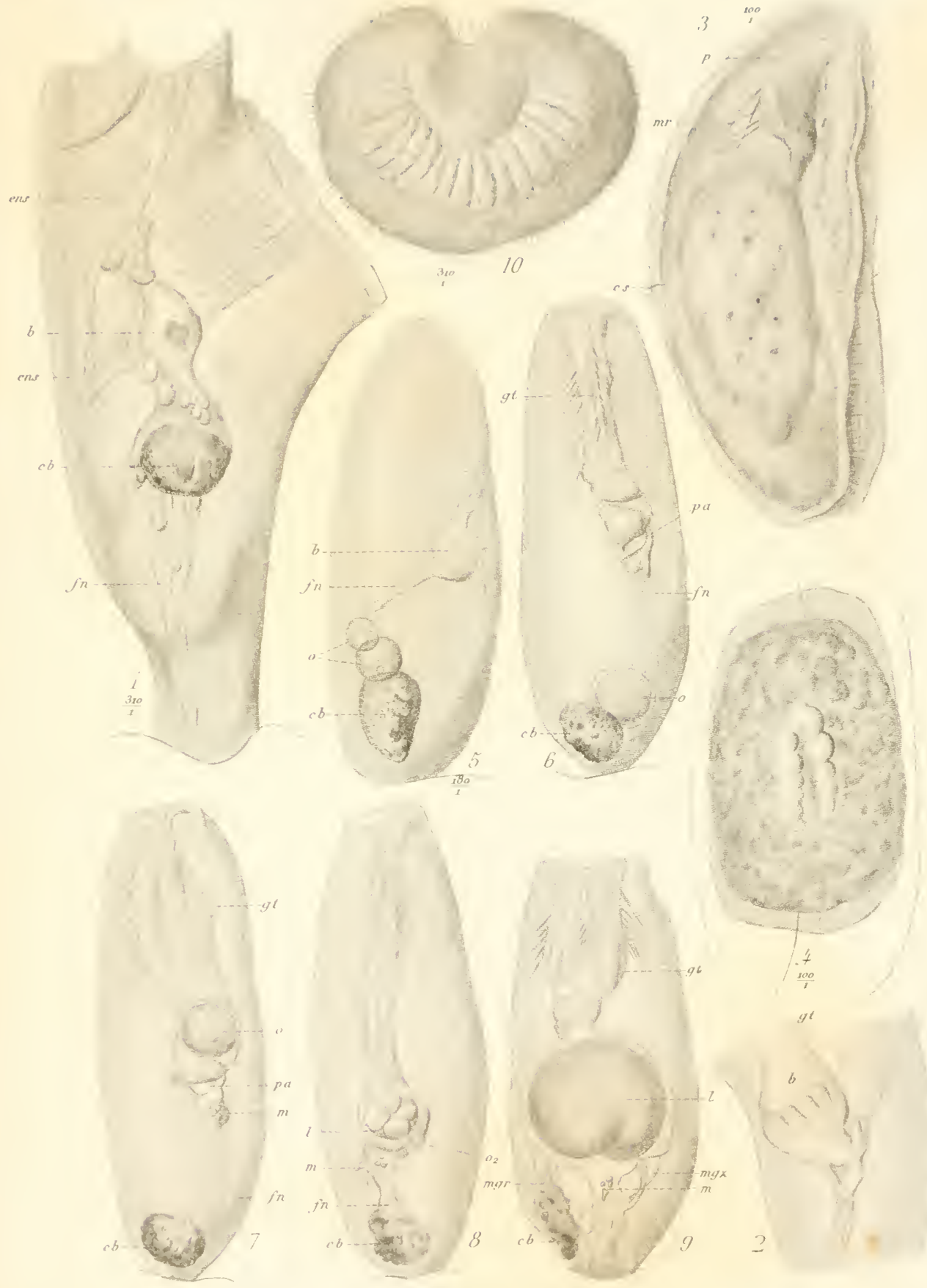

Iny. Th. Chavdsn aune Provis 






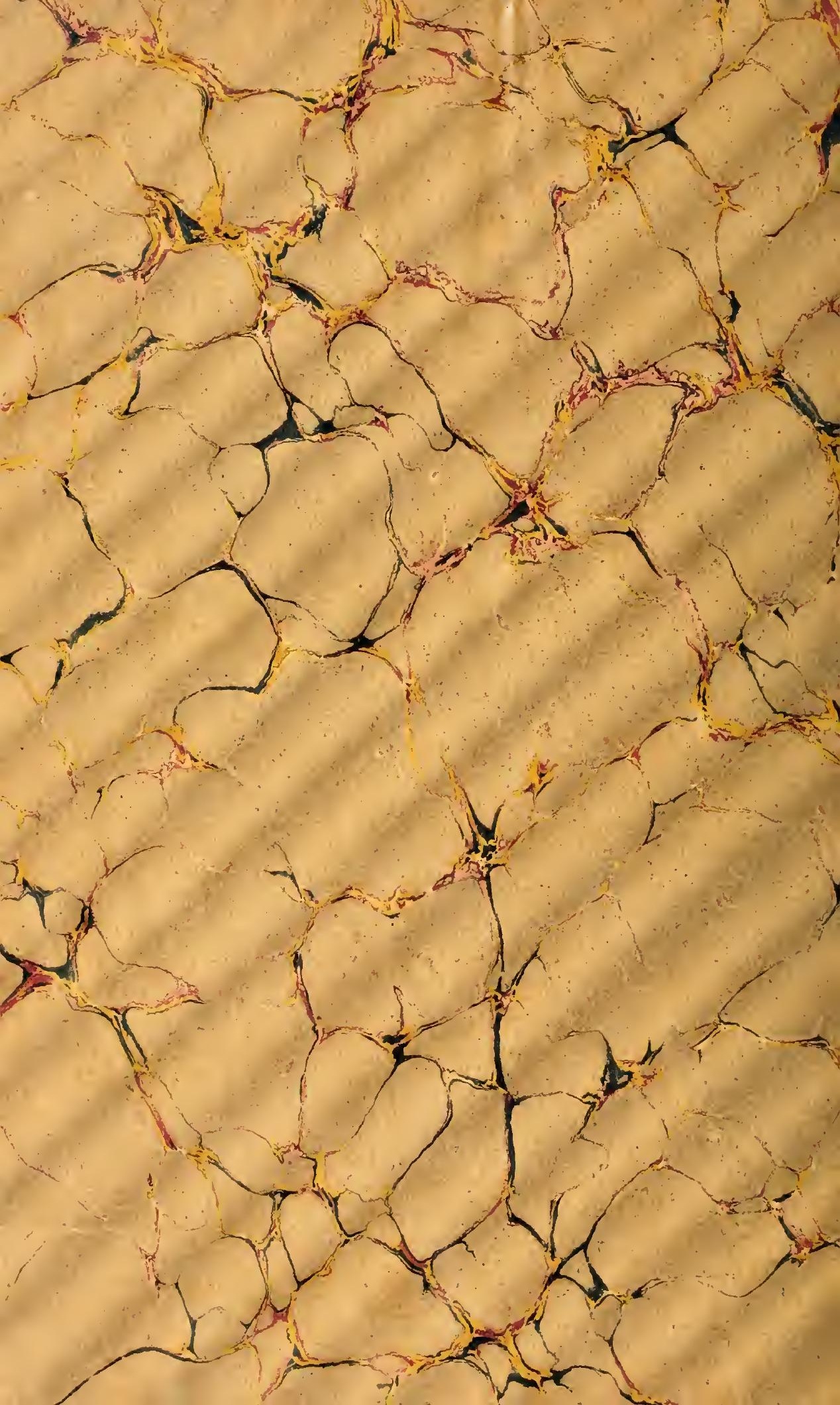




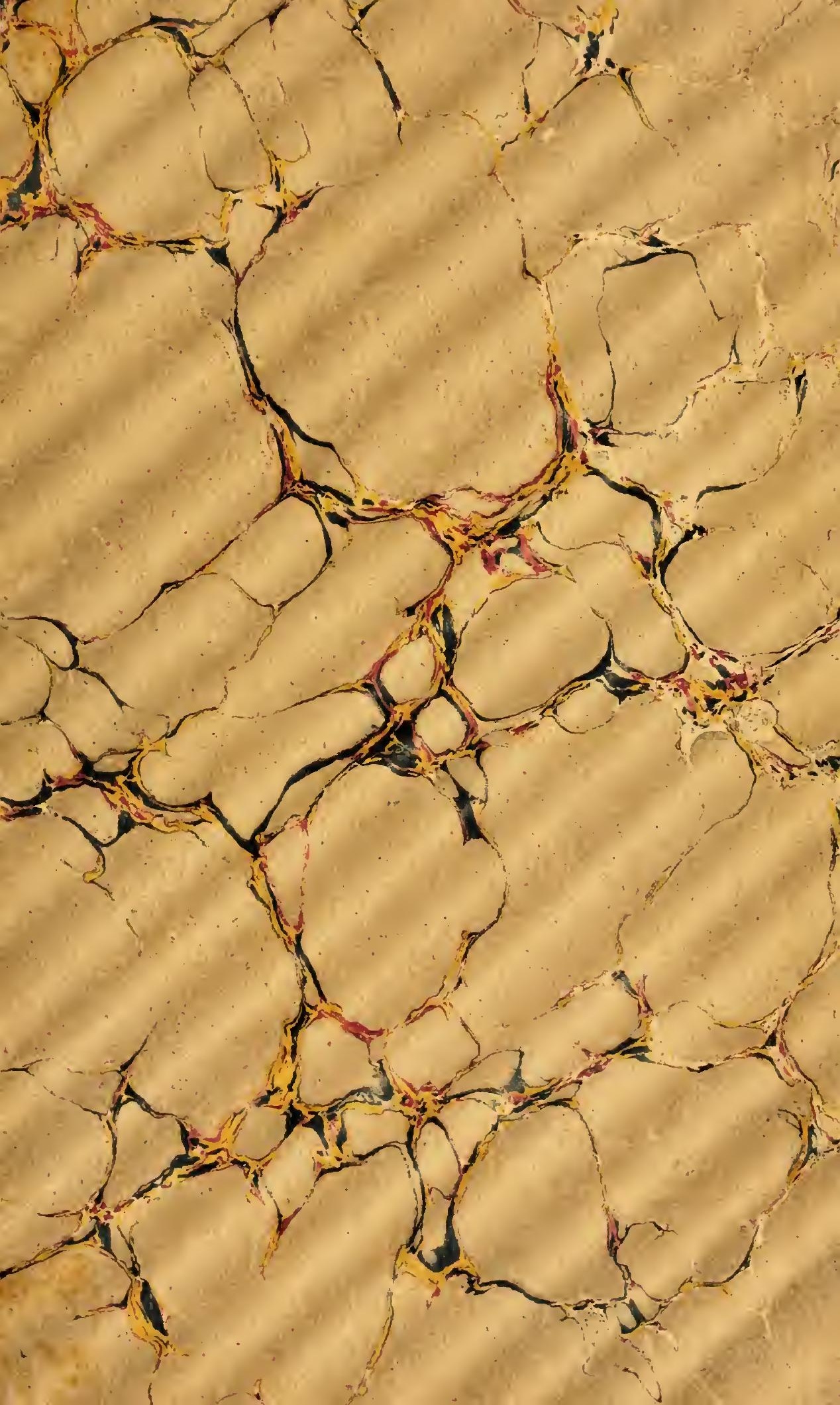




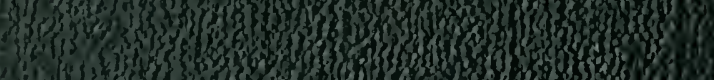

35.10)

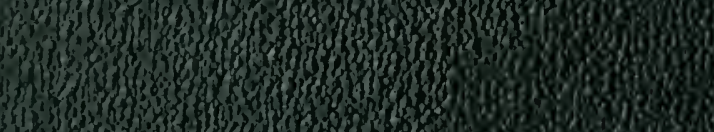

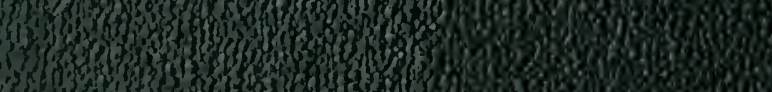

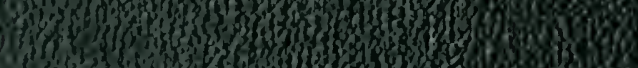

inf?

of

3.

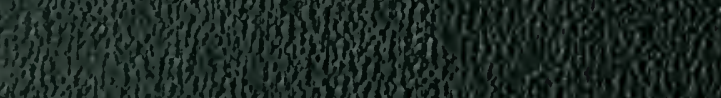

(nj,

If

II

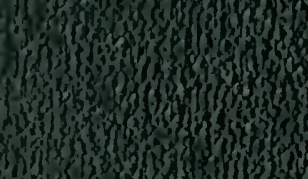

(1). Sul

s.mong

1, wh

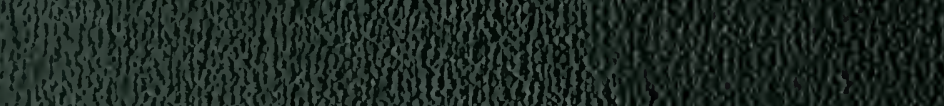

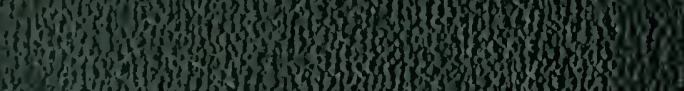

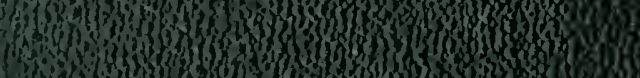

1.in.

1.

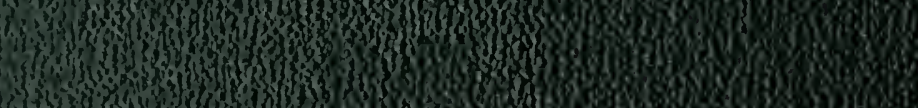

3.

3.in

1) 3 ing

3 s.

i)

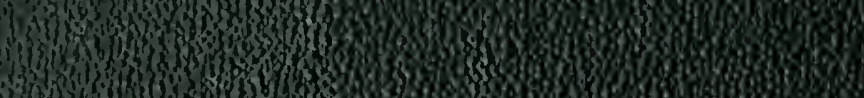
S. n. W. (1) 5 , 3 , Sn 1 in i. 3 , 2.1.

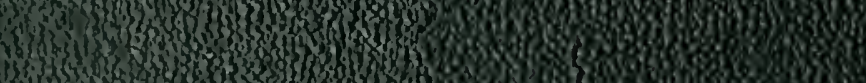
3.

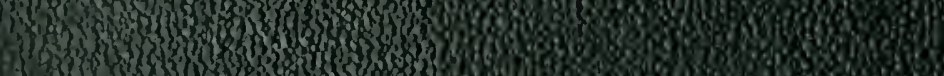

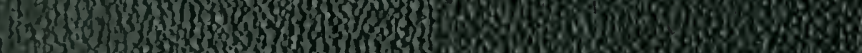

